\title{
Nonlinear structural dynamical system identification using adaptive particle filters
}

\author{
Vikas Namdeo, C.S. Manohar* \\ Department of Civil Engineering, Indian Institute of Science, Bangalore 560 012, India
}

Received 30 January 2007; received in revised form 18 May 2007; accepted 27 May 2007

\begin{abstract}
The problem of identifying parameters of nonlinear vibrating systems using spatially incomplete, noisy, time-domain measurements is considered. The problem is formulated within the framework of dynamic state estimation formalisms that employ particle filters. The parameters of the system, which are to be identified, are treated as a set of random variables with finite number of discrete states. The study develops a procedure that combines a bank of self-learning particle filters with a global iteration strategy to estimate the probability distribution of the system parameters to be identified. Individual particle filters are based on the sequential importance sampling filter algorithm that is readily available in the existing literature. The paper develops the requisite recursive formulary for evaluating the evolution of weights associated with system parameter states. The correctness of the formulations developed is demonstrated first by applying the proposed procedure to a few linear vibrating systems for which an alternative solution using adaptive Kalman filter method is possible. Subsequently, illustrative examples on three nonlinear vibrating systems, using synthetic vibration data, are presented to reveal the correct functioning of the method.

(C) 2007 Elsevier Ltd. All rights reserved.
\end{abstract}

\section{Introduction}

Engineering structures are generally designed to behave linearly and, consequently, linear models for strain-displacement relations, stress-strain laws, and energy dissipation mechanisms could be considered adequate for the purpose of structural analysis, design, and optimization. Concomitant with the development of methods for mathematical modeling and solution of resulting equations of these problems, extensive efforts have been made in the exiting literature to develop methods for identification of structural model parameters, and to reconcile predictions of mathematical and experimental models [1-6]. Within the framework of linear system modeling, issues related to characterizing damping mechanisms at the structure level, joint flexibility, boundary conditions, and material constitutive laws, are intimately connected with experimental investigations. Also, several algorithmic issues related to non-uniqueness of solutions, spatio-temporal incompleteness of response measurement, presence of measurement noise, modeling uncertainties, and difficulties arising out

*Corresponding author. Tel.: +918022933121; fax: +918023600404.

E-mail addresses: vnamdeo@civil.iisc.ernet.in (V. Namdeo), manohar@civil.iisc.ernet.in (C.S. Manohar). 
of complete/partial lack of measurement of inputs have been addressed. The presence of nonlinearity in the structural behavior can lead to a wide ranging phenomenon, such as, multi-valued steady-state responses, jumps, limit cycles, entrainment, internal resonances, super- and sub-harmonic resonances, modal coupling, and complex bifurcations leading to chaos, which a linear structural model would fail to capture. In the context of system identification problems, this calls for development of methods with notable departures from methods used for linear system identification problems. This class of problems has been widely studied in the existing literature and Refs. [7-14] provide extensive overviews of the relevant developments.

Several methods have been developed in time and frequency domains to tackle the problem of nonlinear system identification. The focus of the present study is on methods based on dynamic state estimation within the Bayesian framework. The theory of Kalman filtering provides one of the corner stones in the development of these methods [15-19]. In the context of nonlinear systems, linearization tools, combined with the traditional Kalman filtering techniques, have been developed for dealing with the dynamic state estimation problems [17,20]. It is of interest to note that the problem of state estimation of dynamical systems is widely encountered in many branches of sciences including economics, object tracking, digital communication channels and signal processing. Recent research in these fields has been focused on dealing with nonlinear systems and non-Gaussian noise models. Of particular interest has been the development of the so-called particle filters which employ Monte Carlo simulation procedures for the state estimation problems in dynamical systems [21-30]. These methods are capable of handling general forms of nonlinearities and nonGaussian additive/multiplicative noises. While the roots of these methods date back to 1950s, the recent developments in this field have been spurred by the wide availability of cheap and fast computational power. The applicability of these methods in structural system identification has not yet been explored in the existing literature. A few recent studies have made efforts to remedy this situation. Thus, Manohar and Roy [31] have applied three simulation-based filtering strategies to the problem of system parameter identification in two typical nonlinear oscillators, namely, the Duffing oscillator and the Coulomb oscillator. The filters that these authors investigated included: the density based Monte Carlo filter as developed by Tanizaki [22], the Bayesian bootstrap algorithm due to Gordon et al. [21] and the sequential importance sampling (SIS)-based method as developed by Doucet et al. [26]. Ching et al. [32] have applied stochastic simulation-based filtering technique, namely, the SIS-based method as developed by Doucet et al. [26], and extended Kalman filter (EKF) for identifying parameters of three different classes of dynamical systems: a linear model with time varying system parameters, a nonlinear hysteretic model that can be considered to give moderately nonlinear behavior, and the Lorenz equations that represents a highly nonlinear system. The system parameters to be estimated were treated in this study as auxiliary state variables which are artificially driven by noise processes. These authors have concluded that the particle filters performed satisfactorily for all examples, while the performance of EKF was found to be fair for the second example and poor for the Lorenz system. This is consistent with the expectation that EKF is not suitable for highly nonlinear systems. The problem of identifying spatially localized structural nonlinearities by combining Kalman and particle filtering strategies via the application of the Rao-Blackwell theorem and a time-domain substructuring strategy has been proposed recently by Sajeeb et al. [33]. The application of Bayesian inference approach for model selection in the context of nonlinear dynamical system identification has been considered by Kerschen et al. [34]. Here, a family of parametric models is defined to represent the nonlinear response of a system and the selection algorithm estimates the likelihood that each member of models explored is appropriate. The study is based on the Markov Chain Monte Carlo (MCMC) method in which a Markov chain is constructed such that its equilibrium probability distribution agrees with the target probability distribution from which samples are desired to be drawn.

In the context of identification of linear model parameter identification one of the approaches that has been studied in the existing literature is the so-called adaptive Kalman filtering (also called the self-learning Kalman filtering or the multiple model estimation algorithm). The essence of this method is that the parameters to be identified are treated as a set of discrete random variables with finite states and, for each of the possible states of these random variables, one Kalman filter is employed [17,35]. This results in a bank of filters for the problem on hand and the estimate for the state is obtained as a weighted sum of estimates from individual Kalman filters. The algorithm identifies the member of family of filters which possesses the highest weight and the corresponding value of the unknown system parameter is hypothesized as the estimate of the most probable value of the system parameter. Since the method employs Kalman filters, it is clear that it is 
applicable to only linear systems with Gaussian additive noise and is not directly applicable to problems of nonlinear system identification. In the present study, we extend the scope of adaptive filtering to nonlinear problems by replacing the Kalman filter by a particle filter. By doing so, we systematically extend the scope of adaptive filtering methods to problems of nonlinear structural system identification with, possibly, nonGaussian additive/multiplicative noises. The study first develops the necessary mathematical formulations for this purpose and subsequently illustrates the method proposed by considering three examples involving singleand multi-degree-of-freedom (sdof and mdof) nonlinear systems. As might be expected, if the system on hand is linear and measurement and process noises are Gaussian and additive in nature, the present formulation leads to results similar to those predicted by the adaptive Kalman filter method.

\section{Problem of state estimation}

Consider a mdof system with $N$ dofs governed by

$$
M \ddot{Z}+Q(Z, \dot{Z}, t)=G(t)+\Xi(t) ; Z(0)=Z_{0}, \dot{Z}(0)=\dot{Z}_{0} .
$$

Here a dot represents differentiation with respect to time $t, M$ is the $N \times N$ mass matrix, $Q$ is a $N \times 1$ function of displacement $Z(t)$ and velocity $\dot{Z}(t)$ vectors, $G(t)$ is a $N \times 1$ excitation vector, and $\Xi(t)$ is a $N \times 1$ vector random process representing the process noise that is taken to quantify the unmodeled system dynamics. A set of measurements represented through a $m \times 1$ vector $Y(t)$ is taken to be available and these measurements are related to the system state through the equation

$$
Y(t)=\bar{H}[Z(t), \dot{Z}(t), t]+\Psi(t) .
$$

Here $\Psi(t)$ is a $m \times 1$ vector random process representing the measurement noise. The functions $Q$ and $\bar{H}$ in the above equations are taken to be in general nonlinear and time dependent. The equation of motion (1) can also be expressed in the state space by letting

$$
\{Z(t) \dot{Z}(t)\}^{t}=\left\{\begin{array}{llllllll}
X_{1} & X_{2} & \ldots & X_{N} & X_{1+N} & X_{2+N} & \ldots & X_{2 N}
\end{array}\right\}^{t}
$$

and noting that

$$
\begin{aligned}
\dot{X}_{i} & =X_{i+N}, \\
\dot{X}_{i+N} & =-M^{-1} Q\left(X_{1}, X_{2}, \ldots, X_{2 N}\right)+M^{-1} G(t)+M^{-1} \Xi(t) \\
& =\tilde{F}[X(t), t]+M^{-1} G(t)+M^{-1} \Xi(t) .
\end{aligned}
$$

This can be represented compactly as

$$
\dot{X}(t)=a[X(t), t]+b \Xi(t)
$$

with

$$
\begin{aligned}
& a[X(t), t]=H[X(t), t]+A G(t), \\
& H^{t}=\left\{\begin{array}{llllllll}
X_{1+N} & X_{2+N} & \ldots & X_{2 N} & \tilde{F}_{1} & \tilde{F}_{2} & \ldots & \tilde{F}_{N}
\end{array}\right\}^{t}, \\
& A=\left[\begin{array}{c}
0_{N \times N} \\
M^{-1}
\end{array}\right]_{(2 N \times N)} b=\left[\begin{array}{c}
0_{N \times N} \\
M^{-1}
\end{array}\right]_{(2 N \times N)} .
\end{aligned}
$$

If the random processes $\Xi(t)$ and $\Psi(t)$ are modeled as zero mean Gaussian white noise processes obtained as formal derivatives of Weiner processes, we can represent the process equation (4) as an stochastic differential equations as

$$
\mathrm{d} X(t)=a[X(t), t] \mathrm{d} t+b \sigma \mathrm{d} B(t), \quad X(0)=X_{0} .
$$

Here $\mathrm{d} B(t)$ is a $N \times 1$ vector of increments of Brownian motion processes and $\sigma$ is the $N \times N$ covariance matrix.

For the purpose of dynamic state estimation, we need to discretize the measurement and process equations in time. The discretization of measurement equation is naturally dictated by the fact that the measurements 
$Y(t)$ are made at discrete time instants $t=t_{k}=k h ; k=1,2, \ldots, N_{k}$ where $h$ is the time step at which measurement signal is sampled. This leads to the measurement equation of the general form

$$
y_{k}=f_{k}\left(x_{k}\right)+q_{k}\left(x_{k}\right) v_{k},
$$

where $v_{k}$ for $k=1,2, \ldots, N_{k}$ is a sequence of independent Gaussian random variables. In order to discretize the process equation we employ the methods available in the existing literature for the numerical solution of stochastic differential equations. It is assumed that the time step used in discretization is identical to the time step used in sampling the response waveform during the measurement. It is emphasized that this is not a restrictive assumption and a general formulation with differing step sizes for measurement and discretization of the process equation can be easily developed. The details of the discretization scheme used and the choice of step size $h$ should be such that acceptable convergence in a strong sense is secured: the book by Kloeden and Platen [36] provides extensive account of these considerations. Details as relevant to the present study are available in the work of Manohar and Roy [31]. The process equation for further consideration is now written in the form

$$
x_{k+1}=h_{k}\left(x_{k}\right)+\gamma_{k} w_{k}, \quad k=0,1, \ldots, N_{k} .
$$

with specified $x_{0}$. The quantities $\left\{w_{k}\right\}_{k=1}^{N_{k}}$ are taken to be a set of mutually independent Gaussian random variables with specified variance. This, again, is not a restrictive assumption. Furthermore, these random variables are taken to be uncorrelated with the random variables $\left\{v_{k}\right\}_{k=1}^{N_{k}}$ that appear in the measurement equation. This again, is not a restrictive assumption. The quantities $x_{k}, h_{k}, \gamma_{k}$ and $w_{k}$ appearing in the process equation (9) are vectors of sizes $2 N \times 1,2 N \times 1,2 N \times N$ and $N \times 1$, respectively. Similarly, the quantities $y_{k}, f_{k}$, and $v_{k}$ appearing in the measurement equation (8) are vectors of size $m \times 1, m \times m$ and $m \times m$ respectively. We denote by $\Sigma_{w}$ and $\Sigma_{v}$ respectively the covariance matrix of the vectors $w_{k}$ and $v_{k}$. The quantities $\left\{x_{k}\right\}_{k=0}^{N_{k}}$ and $\left\{y_{k}\right\}_{k=1}^{N_{k}}$ constitute a set of vector random variables. In the discussion to follow we deviate from the standard practice of notationally distinguishing a random variable $X$ from its state $x$ and employ the same notation $x$ to denote both the random variables and their respective states. This affords a significant simplification in presenting the mathematical formulation and is consistent with the practice followed in literature related to dynamic state estimation methods.

\section{Formal solution to the state estimation problem}

We introduce the notations $x_{0: k}$ and $y_{1: k}$ to respectively denote

$$
\begin{aligned}
x_{0: k} & =\left\{\begin{array}{llll}
x_{0} & x_{1} & \ldots & x_{k}
\end{array}\right\}, \\
y_{1: k} & =\left\{\begin{array}{llll}
y_{1} & y_{2} & \ldots & y_{k}
\end{array}\right\} .
\end{aligned}
$$

It is assumed that the probability density function (pdf) $p\left(x_{0}\right)$ is specified and, also, it follows that $p\left(x_{k} \mid x_{k-1}\right)$ and $p\left(y_{k} \mid x_{k}\right)$ are determinable in terms of known properties of the noise terms $w_{k}$ and $v_{k}$. In the present case, these pdfs turn out to be Gaussian in nature. The problem of state estimation consists of determining the posterior pdf $p\left(x_{0: k} \mid y_{1: k}\right)$ and the filtering density function $p\left(x_{k} \mid y_{1: k}\right)$. Also of interest is the determination of the moments

$$
\begin{aligned}
a_{k \mid k} & =E\left[x_{k} \mid y_{1: k}\right]=\int x_{k} p\left(x_{k} \mid y_{1: k}\right) \mathrm{d} x_{k}, \\
\sum_{k \mid k} & =E\left[\left(x_{k}-a_{k \mid k}\right)\left(x_{k}-a_{k \mid k}\right)^{t}\right]=\int\left(x_{k}-a_{k \mid k}\right)\left(x_{k}-a_{k \mid k}\right)^{t} p\left(x_{k} \mid y_{1: k}\right) \mathrm{d} x_{k} .
\end{aligned}
$$

Here $E[\cdot]$ denotes the mathematical expectation operator and a superscript $t$ denote the matrix transposition. The integrals appearing in the right-hand sides are $2 N$ dimensional and are evaluated over the limits $(-\infty, \infty)$ : these features are not displayed explicitly in the above equations to simplify the presentation of the equations. Formal solutions for the state estimation problem are available in the literature. For the determination of filtering density, the formal solution consists of a pair of prediction and updation 
equations can be shown to be given, respectively, by

$$
\begin{aligned}
p\left(x_{k} \mid y_{1: k-1}\right) & =\int p\left(x_{k} \mid x_{k-1}\right) p\left(x_{k-1} \mid y_{1: k-1}\right) \mathrm{d} x_{k-1}, \\
p\left(x_{k} \mid y_{1: k}\right) & =\frac{p\left(y_{k} \mid x_{k}\right) p\left(x_{k} \mid y_{1: k-1}\right)}{\int p\left(y_{k} \mid x_{k}\right) p\left(x_{k} \mid y_{1: k-1}\right) \mathrm{d} x_{k}} .
\end{aligned}
$$

A recursive equation for the evolution of the multidimensional posteriori pdf can also be obtained as [26]

$$
p\left(x_{0: k+1} \mid y_{1: k+1}\right)=p\left(x_{0: k} \mid y_{1: k}\right) \frac{p\left(y_{k+1} \mid x_{k+1}\right) p\left(x_{k+1} \mid x_{k}\right)}{p\left(y_{k+1} \mid y_{1: k}\right)} .
$$

The actual solutions of the above equations by using numerical quadrature are, however, infeasible because of the unwieldy dimensions of the integrals involved. For the particular case of linear process and measurement equations and Gaussian additive noises, the above equations lead to the well-known Kalman filter. For a certain class of nonlinear process equations, linear measurement equations and additive Gaussian noises, the problem of state estimation admits an exact solution [30]. For more general forms of process and measurement equations, additive or multiplicative noises and Gaussian/non-Gaussian noises, several alternative strategies have been developed. One set of such alternatives consist of developing suboptimal filtering strategies, such as those based on linearization or transformations, and the other consists of methods which employ Monte Carlo simulation strategies to evaluate the multidimensional integrals in a recursive manner. It is the latter class of approaches that are being considered in the present study.

\section{System parameter identification by a bank of filters}

We define $\theta$ to be an $n \times 1$ vector of system parameters that need to be identified. In order to show the dependence of the system equations on this vector, we re-write the process and measurement equations, respectively, as follows:

$$
\begin{aligned}
x_{k+1} & =h_{k}\left(x_{k}, \theta\right)+\gamma_{k} w_{k}, \quad k=0,1,2, \ldots, N_{k}, \\
y_{k} & =f_{k}\left(x_{k}, \theta\right)+q_{k}\left(x_{k}, \theta\right) v_{k}, \quad k=1,2, \ldots, N_{k} .
\end{aligned}
$$

In the problems of system identification one needs to determine the pdf of the system parameters $\theta$. One of the strategies that has been employed in the existing literature is to declare the vector $\theta$ to be an additional state vector and augment the process equation with additional artificial equations of the form $\mathrm{d} \theta(t)=\mathrm{d} \tilde{B}(t), \theta(0)=\theta_{0}$ or, equivalently in a discretized form as $\theta_{k+1}=\theta_{k}+\zeta_{k}, k=0,1,2, \ldots, N_{k}$. The augmented state vector in this case would be $(2 N+n) \times 1$ dimensional. The resulting problem could then be studied using either the suboptimal filtering strategies or by using the Monte Carlo filters. In the context of particle filtering methods, this approach has been studied by Gordon et al. [21] and also by Liu and West [37]. The recent studies by Manohar and Roy [31], and Ching et al. [32], have explored the particle filtering strategy in the context of identification of parameters of a class of nonlinear oscillators. An alternative strategy that avoids treating unknown parameters as additional state variables consists of developing filters that adapt themselves to unknown system parameters [17,35]. Here the unknown system parameters are viewed as a set of random variables and the problem of state estimation is construed as determining

$$
\begin{aligned}
a_{k \mid k} & =\int x_{k} \int p\left(x_{k}, \theta \mid y_{1: k}\right) \mathrm{d} \theta \mathrm{d} x_{k} \\
& =\int x_{k} \int p\left(x_{k} \mid \theta, y_{1: k}\right) p\left(\theta \mid y_{1: k}\right) \mathrm{d} \theta \mathrm{d} x_{k} \\
& =\int p\left(\theta \mid y_{1: k}\right)\left[\int_{x_{k}} x_{k} p\left(x_{k} \mid \theta, y_{1: k}\right) \mathrm{d} x_{k}\right] \mathrm{d} \theta \\
& =\int p\left(\theta \mid y_{1: k}\right) \hat{x}_{k}(\theta) \mathrm{d} \theta .
\end{aligned}
$$


If we postulate the parameter $\theta$ to be a vector of discrete random variables with $L$ number of states, then Eq. (15) can be re-written as

$$
a_{k \mid k}=\sum_{i=1}^{L} \hat{x}_{k}\left(\theta_{i}\right) p\left(\theta_{i} \mid y_{1: k}\right) .
$$

Here $p\left(\theta_{i} \mid y_{1: k}\right)$ is the probability mass function given by $p\left(\theta_{i} \mid y_{1: k}\right)=p\left[\theta=\theta_{i} \mid y_{1: k}\right]$. The problem on hand consists of determining the weights $p\left(\theta_{i} \mid y_{1: k}\right)$ and the estimate $\hat{x}_{k}\left(\theta_{i}\right)$. Clearly the value of the weights $p\left(\theta_{i} \mid y_{1: k}\right)$ would be influenced by the measurement $y_{1: k}$ and, as the measurements are assimilated, the adaptive scheme learns which of the filters are the correct ones, and its weight factor $p\left(\theta_{i} \mid y_{1: k}\right)$ approaches unity while the others are going to zero. This would mean that the filter becomes adaptive or self-learning in nature as the measurement is assimilated into the state estimation problem. The solution procedure thus would consist of running $L$ filters, each for a specific value of $\theta=\theta_{i}$, with $i=1,2, \ldots, L$, and trace the evolution of the weights $p\left(\theta_{i} \mid y_{1: k}\right)$ as measurements are assimilated into the filtering process. Based on Bayes' rule we write

$$
p\left(\theta_{i} \mid y_{1: k}\right)=\frac{p\left(y_{1: k} \mid \theta_{i}\right) p\left(\theta_{i}\right)}{p\left(y_{1: k}\right)}=\frac{p\left(y_{1: k} \mid \theta_{i}\right) p\left(\theta_{i}\right)}{\sum_{j=1}^{L} p\left(y_{1: k} \mid \theta_{j}\right) p\left(\theta_{j}\right)}, \quad i=1,2, \ldots, L .
$$

The a priori distribution $p\left(\theta_{i}\right)$ is assumed to be known. Thus, the implementation of the algorithm requires an initial hypothesis to be made on the pdf $P\left[\theta=\theta_{i}\right] ; i=1,2, \ldots, L$. A reasonable guess would be to take this distribution to correspond to a uniform distribution over an hypothesized range of values of $\theta$. Fig. 1 shows the schematic of the strategy for the implementation of the bank of filters. When the process and measurement equations are linear and noises are Gaussian and additive, the estimate $\hat{x}_{k}\left(\theta_{i}\right)$ could be obtained exactly using the standard Kalman filter. For more general class of problems involving nonlinearities, non-Gaussian noises and/or multiplicative action of noises, the estimation of $\hat{x}_{k}\left(\theta_{i}\right)$ could be based on the application of particle filters. The details of evaluation of the weights $p\left(\theta_{i} \mid y_{1: k}\right)$ in these two cases would be accordingly different. We consider the latter case in this study and refer to the work of Brown and Hwang [17] for details of the Kalman filter-based solution. It may be noted that given the knowledge of $p\left(\theta_{i} \mid y_{1: k}\right)$, the posteriori expected values of functions of $\theta$ could be determined by using

$$
E\left[f(\theta) \mid y_{1: k}\right]=\sum_{j=1}^{L} f\left(\theta_{j}\right) p\left(\theta_{j} \mid y_{1: k}\right) .
$$

Using this equation, for instance, we can determine the mean and standard deviation of $\theta$.

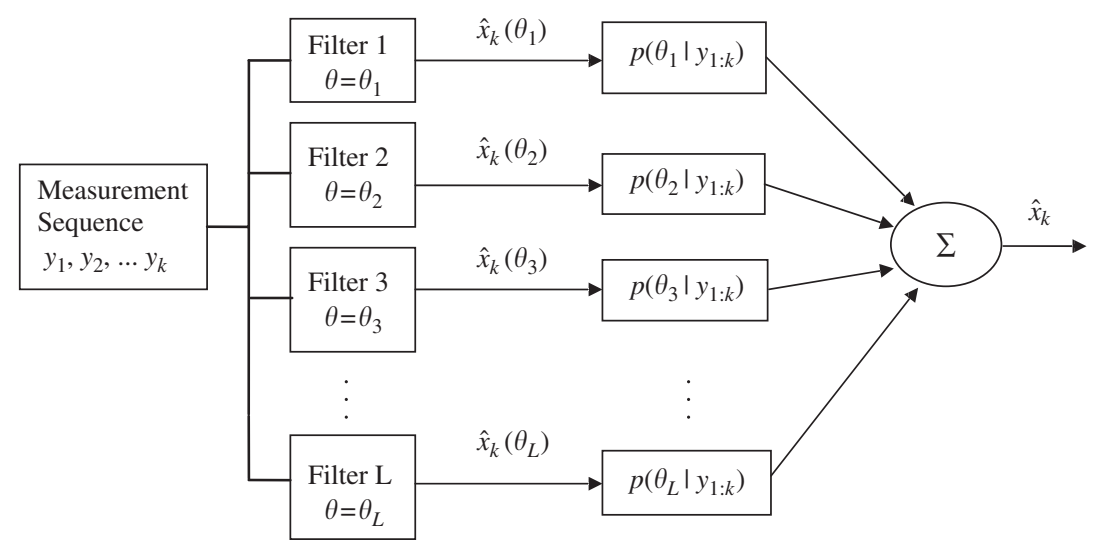

Fig. 1. Bank of filters for system identification. 


\subsection{Estimation of $\hat{x}_{k}\left(\theta_{i}\right)$ using particle filters and the determination of the weights $p\left(\theta_{i} \mid y_{1: k}\right)$}

Here we consider the general governing equations as in Eq. (14). Apart from the fact that the functions $h_{k}\left(x_{k}, \theta\right), f_{k}\left(x_{k}, \theta\right)$ and $q_{k}\left(x_{k}, \theta\right)$ could be nonlinear in the state vector $x_{k}$, it may also be noted that the random variable vectors, $w_{k}$ and $v_{k}$, in principle, could be non-Gaussian in nature. This possibility, however, has not been explored in the present study and we have restricted our attention to Gaussian models for $w_{k}$ and $v_{k}$. The problem of determining the states $\hat{x}_{k}\left(\theta_{i}\right)$ and the weights $p\left(\theta_{i} \mid y_{1: k}\right)$ is not amenable for solution by the Kalman filter method and we resort to the particle filter strategy to solve the problem. Accordingly, for the estimation of $\hat{x}_{k}\left(\theta_{i}\right)$ we use the sequential importance sampling (SIS) particle filters as proposed by Doucet [23] (see Appendix A for an outline of the steps) and for the estimation of the weights $p\left(\theta_{i} \mid y_{1: k}\right)$ we present a strategy that conforms to the aspirations of the particle filter strategy. The contribution of the present work essentially consists of imbedding the procedure for the determination of $\hat{x}_{k}\left(\theta_{i}\right)$ and $p\left(\theta_{i} \mid y_{1: k}\right)$ into a recursive format which leads to the solution of the problem of system parameter identification.

The problem on hand consists of determining the posteriori pdf $p\left(x_{0: k} \mid y_{1: k}\right)$, its associated marginal $p\left(x_{k} \mid y_{1: k}\right)$ and (or) expectations of the form

$$
I\left[x_{0: k}\right]=E_{p}\left[x_{0: k} \mid y_{1: k}\right]=\int_{x} x_{0: k} p\left(x_{0: k} \mid y_{1: k}\right) \mathrm{d} x_{0: k} .
$$

Recognizing the presence of the unknown vector $\theta$ in Eq. (14), we re-write the above equation as

$$
\begin{aligned}
I\left[x_{0: k}\right] & =\int_{x} x_{0: k} \int_{\theta} p\left(x_{0: k}, \theta \mid y_{1: k}\right) \mathrm{d} \theta \mathrm{d} x_{0: k} \\
& =\int_{x} x_{0: k} \int_{\theta} p\left(x_{0: k} \mid \theta, y_{1: k}\right) p\left(\theta \mid y_{1: k}\right) \mathrm{d} \theta \mathrm{d} x_{0: k} \\
& =\int_{\theta} p\left(\theta \mid y_{1: k}\right) \int_{x} x_{0: k} p\left(x_{0: k} \mid \theta, y_{1: k}\right) \mathrm{d} x_{0: k} \mathrm{~d} \theta \\
& =\int_{\theta} \hat{I}\left[x_{0: k}(\theta)\right] p\left(\theta \mid y_{1: k}\right) \mathrm{d} \theta .
\end{aligned}
$$

Now, if we postulate that the parameter $\theta$ to be a vector of discrete random variables with $L$ number of states, then Eq. (20) can be re-written as

$$
I\left[x_{0: k}\right]=\sum_{i=1}^{L} \hat{I}\left[x_{0: k}\left(\theta_{i}\right)\right] p\left(\theta_{i} \mid y_{1: k}\right) .
$$

Here, $p\left(\theta_{i} \mid y_{1: k}\right)$ is the probability mass function given by $p\left(\theta_{i} \mid y_{1: k}\right)=p\left[\theta=\theta_{i} \mid y_{1: k}\right]$. The problem on hand consists of determining the weights $p\left(\theta_{i} \mid y_{1: k}\right)$ and the estimate $\hat{I}\left[x_{0: k}\left(\theta_{i}\right)\right]$. We begin by noting the identities

$$
\begin{aligned}
p\left(\theta \mid y_{1: k}\right) & =\frac{p\left(\theta, y_{1: k}\right)}{p\left(y_{1: k}\right)}=\frac{\int p\left(\theta, y_{1: k}, x_{0: k}\right) \mathrm{d} x_{0: k}}{\int p\left(y_{1: k}, x_{0: k}\right) \mathrm{d} x_{0: k}}=\frac{\int p\left(y_{1: k}, x_{0: k} \mid \theta\right) p(\theta) \mathrm{d} x_{0: k}}{\int_{\theta} \int_{x_{0: k}} p\left(y_{1: k}, x_{0: k} \mid \theta\right) p(\theta) \mathrm{d} x_{0: k} \mathrm{~d} \theta} \\
& =\frac{\int p\left(y_{1: k} \mid x_{0: k}, \theta\right) p\left(x_{0: k} \mid \theta\right) p(\theta) \mathrm{d} x_{0: k}}{\int_{\theta} \int_{x_{0: k}} p\left(y_{1: k} \mid x_{0: k}, \theta\right) p(\theta) p\left(x_{0: k} \mid \theta\right) \mathrm{d} x_{0: k} \mathrm{~d} \theta} .
\end{aligned}
$$

We note from Eq. (14) that

$$
\begin{aligned}
p\left(y_{1: k} \mid x_{0: k}, \theta\right) & =\prod_{j=1}^{k} p\left(y_{j} \mid x_{j}, \theta\right), \\
p\left(x_{0: k} \mid \theta\right) & =p\left(x_{0}\right) \prod_{j=1}^{k} p\left(x_{j} \mid x_{j-1}, \theta\right) .
\end{aligned}
$$


Substituting this in Eq. (22) we obtain

$$
\begin{gathered}
p\left(\theta \mid y_{1: k}\right)=\frac{\int_{x_{0: k}} p\left(x_{0}\right) \prod_{i=1}^{k} p\left(y_{i} \mid x_{i}, \theta\right) p\left(x_{i} \mid x_{i-1}, \theta\right) p(\theta) \mathrm{d} x_{0: k}}{\int_{\theta} \int_{x_{0: k}} p\left(x_{0}\right) \prod_{i=1}^{k} p\left(y_{i} \mid x_{i}, \theta\right) p\left(x_{i} \mid x_{i-1}, \theta\right) p(\theta) \mathrm{d} x_{0: k} \mathrm{~d} \theta}, \\
p\left(\theta \mid y_{1: k}\right)=\frac{\left[\int_{x_{0: k-1}} p\left(x_{0}\right) \prod_{i=1}^{k-1} p\left(y_{i} \mid x_{i}, \theta\right) p\left(x_{i} \mid x_{i-1}, \theta\right) \mathrm{d} x_{0: k-1}\right]\left[\int_{x_{k}} p\left(y_{k} \mid x_{k}, \theta\right) p\left(x_{k} \mid x_{k-1}, \theta\right) \mathrm{d} x_{k}\right] p(\theta)}{\int_{\theta} p(\theta)\left[\int_{x_{0: k-1}} p\left(x_{0}\right) \prod_{i=1}^{k-1} p\left(y_{i} \mid x_{i}, \theta\right) p\left(x_{i} \mid x_{i-1}, \theta\right) \mathrm{d} x_{0: k-1}\right]\left[\int_{x_{k}} p\left(y_{k} \mid x_{k}, \theta\right) p\left(x_{k} \mid x_{k-1}, \theta\right) \mathrm{d} x_{k}\right] \mathrm{d} \theta} .
\end{gathered}
$$

Now, as before, if we postulate that the parameter $\theta$ to be a vector of discrete random variables with $L$ number of states, then Eq. (24) can be re-written as

$$
\begin{aligned}
p\left(\theta_{j} \mid y_{1: k}\right)= & \frac{\left[\int_{x_{0: k-1}} p\left(x_{0}\right) \prod_{i=1}^{k-1} p\left(y_{i} \mid x_{i}, \theta_{j}\right) p\left(x_{i} \mid x_{i-1}, \theta_{j}\right) \mathrm{d} x_{0: k-1}\right]\left[\int_{x_{k}} p\left(y_{k} \mid x_{k}, \theta_{j}\right) p\left(x_{k} \mid x_{k-1}, \theta_{j}\right) \mathrm{d} x_{k}\right] p\left(\theta_{j}\right)}{\sum_{m=1}^{L} \int_{\theta} p(\theta)\left[\int_{x_{0: k-1}} p\left(x_{0}\right) \prod_{i=1}^{k-1} p\left(y_{i} \mid x_{i}, \theta\right) p\left(x_{i} \mid x_{i-1}, \theta\right) \mathrm{d} x_{0: k-1}\right]\left[\int_{x_{k}} p\left(y_{k} \mid x_{k}, \theta\right) p\left(x_{k} \mid x_{k-1}, \theta\right) \mathrm{d} x_{k}\right] \delta\left(\theta-\theta_{m}\right) \mathrm{d} \theta} \\
& j=1,2, \ldots, L .
\end{aligned}
$$

This equation can be recast in a recursive format as follows:

$$
p\left(\theta_{j} \mid y_{1: k}\right)=\frac{\left[\int_{x_{k}} p\left(y_{k} \mid x_{k}, \theta_{j}\right) p\left(x_{k} \mid x_{k-1}, \theta_{j}\right) \mathrm{d} x_{k}\right] p\left(\theta_{j}\right)}{\sum_{m=1}^{L}\left[\int_{x_{k}} p\left(y_{k} \mid x_{k}, \theta_{m}\right) p\left(x_{k} \mid x_{k-1}, \theta_{m}\right) \mathrm{d} x_{k}\right] p\left(\theta_{m}\right)} p\left(\theta_{j} \mid y_{1: k-1}\right) .
$$

The multidimensional integrals appearing in the above equation are not amenable for evaluation in closed form and we propose to estimate their values using Monte Carlo simulation. Accordingly we draw $N$ samples from the pdf $p\left(x_{k} \mid x_{k-1}, \theta_{j}\right)$ and obtain

$$
p\left(\theta_{j} \mid y_{1: k}\right)=\frac{\sum_{n=1}^{N} p\left(y_{k} \mid x_{n, k}, \theta_{j}\right) p\left(\theta_{j}\right)}{\sum_{m=1}^{L}\left[\sum_{n=1}^{N} p\left(y_{k} \mid x_{n, k}, \theta_{m}\right) p\left(\theta_{m}\right)\right]} p\left(\theta_{j} \mid y_{1: k-1}\right) .
$$

Here it must be noted that the samples from $p\left(x_{k} \mid x_{k-1}, \theta_{j}\right)$ are obtained by using the process equation (14a). Also, as has been already noted, $p\left(y_{k} \mid x_{k}, \theta_{j}\right)$ is determinable from the measurement equation (14b).

\subsubsection{Remarks}

1. As the measurement is assimilated into the filtering process, the weight factor corresponding to the true value of system parameter $\theta$ approaches the value of unity with other weights going to zero.

2. The range of initial pdf $p(\theta)$ is expected to include the true value of $\theta$. This can only be ensured based on engineering judgment.

3. In an online application, the number of discrete states for $\theta$, namely, $L$, must be such that satisfactory resolution on the value of $\theta$ is achieved. On the other hand, if the identification process is to be implemented off-line, one could begin with coarser division of range of $\theta$ and run the bank of filters to identify the interval over which true $\theta$ is likely to lie. This interval alone can be refined in a further implementation of next cycle of bank of filters. This procedure permits determination of $\theta$ to a desired level of accuracy and we have adopted this strategy in the present paper. 


\section{Numerical illustrations}

In the illustrative examples considered in this section the measurements are synthetically simulated and seeded by random numbers to simulate the effect of measurement noise. Figs. 2-5 show the different examples considered in this study.

\subsection{Example 1: Linear single degree of freedom oscillator}

We consider the single degree of freedom (sdof) system driven by support displacement $x_{s}(t)$ as shown in Fig. 2. The governing equation of motion for this system in terms of the relative displacement, $x_{1}(t)$, and

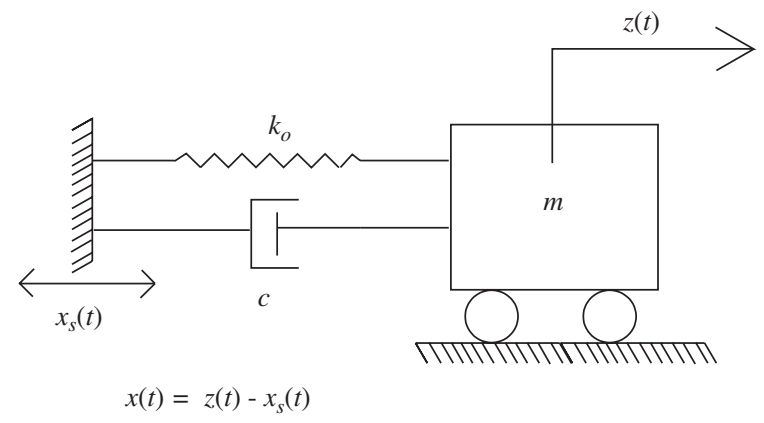

Fig. 2. sdof Spring mass damper system.

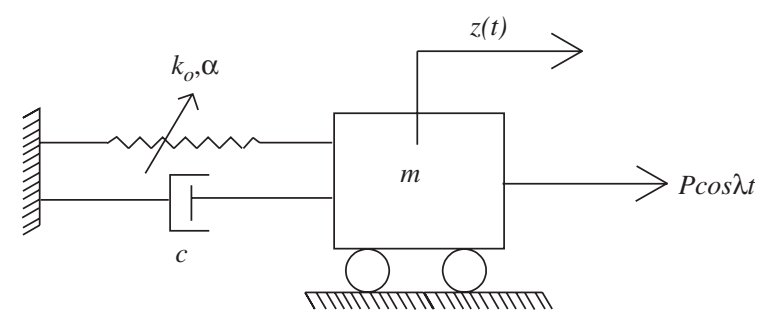

Fig. 3. sdof Duffing's oscillator.

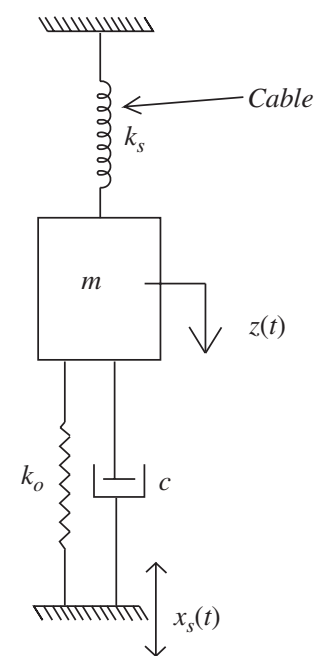

Fig. 4. Nonlinear sdof system with bilinear stiffness characteristics. 


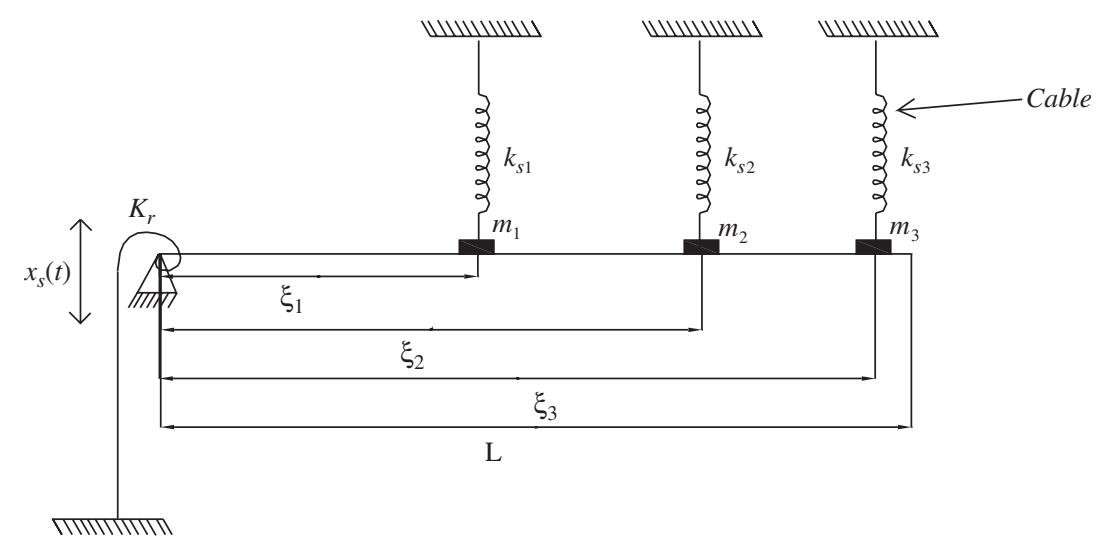

Fig. 5. Euler-Bernoulli beam suspended on three cables that offer resistance only to tensile forces.

relative velocity, $x_{2}(t)$, is given by

$$
\begin{aligned}
\mathrm{d} x_{1} & =x_{2} \mathrm{~d} t, \\
\mathrm{~d} x_{2} & =\left[-\frac{c}{m} x_{2}-\frac{k_{o}}{m} x_{1}-\ddot{x}_{s}(t)\right] \mathrm{d} t+\sigma \mathrm{d} B(t), \\
x_{1}(0) & =0 ; x_{2}(0)=0 .
\end{aligned}
$$

For the purpose of illustration we take the support displacement to be a sample of a stationary random process generated using the Fourier representation

$$
x_{s}(t)=\sum_{j=1}^{n} \sigma_{j}\left[V_{j} \cos \omega_{j} t+W_{j} \sin \omega_{j} t\right] .
$$

Here, $\left\{V_{j}\right\}_{j=1}^{n}$ and $\left\{W_{j}\right\}_{j=1}^{n}$ are taken to be identical, independently distributed Gaussain random variables with zero mean unit standard deviations. It is also assumed that $V_{j}$ and $W_{k}$ are independent for all $j$ and $k$. The constants $\left\{\sigma_{j}\right\}_{j=1}^{n}$ and $\omega_{j}=j \omega_{0}$ are taken to be specified and deterministic. The expression for support velocity and acceleration are obtained as

$$
\begin{aligned}
& \dot{x}_{s}(t)=\sum_{j=1}^{n} \sigma_{j} \omega_{j}\left[-V_{j} \sin \omega_{j} t+W_{j} \cos \omega_{j} t\right], \\
& \ddot{x}_{s}(t)=\sum_{j=1}^{n}-\sigma_{j} \omega_{j}^{2}\left[V_{j} \cos \omega_{j} t+W_{j} \sin \omega_{j} t\right] .
\end{aligned}
$$

It is to be emphasized that in our study we use only a single realization of $x_{s}(t)$ although it is drawn as a sample of a stationary Gaussian random process. A discretized version of Eq. (28) is obtained using Ito-Taylor expansion and is given by

$$
\begin{aligned}
x_{1(k+1)}= & x_{1 k}+x_{2 k} h+\left(-\frac{k_{o}}{m} x_{1 k}-\frac{c}{m} x_{2 k}\right) \frac{h^{2}}{2}-\ddot{x}_{s k} \frac{h^{2}}{2}+\frac{\sigma}{m} I_{10}, \\
x_{2(k+1)}= & x_{2 k}+\left(-\frac{k_{o}}{m} x_{1 k}-\frac{c}{m} x_{2 k}\right) h-\frac{k_{o}}{m} x_{2 k} \frac{h^{2}}{2}+\frac{c k_{o}}{m^{2}} x_{1 k} \frac{h^{2}}{2}+\frac{c^{2}}{m^{2}} x_{2 k} \frac{h^{2}}{2} \\
& +\frac{c}{m} \ddot{x}_{s k} \frac{h^{2}}{2}-\frac{c}{m^{2}} \sigma I_{10}+\frac{\sigma}{m} I_{1}-v_{s k},
\end{aligned}
$$


with $v_{s k}=\dot{x}_{s(k+1)}-\dot{x}_{s k}$. The quantities $I_{1}$ and $I_{10}$ are the multiple stochastic integrals which constitute a pair of Gaussian random variables such that

$$
\left\{\begin{array}{c}
I_{1} \\
I_{10}
\end{array}\right\} \equiv N\left[0_{2 \times 1},\left[\begin{array}{cc}
h & \frac{h^{2}}{2} \\
\frac{h^{2}}{2} & \frac{h^{3}}{3}
\end{array}\right]\right] .
$$

It is assumed that the measurements on the total displacement and velocity of the system are obtained and the measurement equations are written as

$$
\begin{aligned}
& y_{1 k}=x_{1 k}+x_{s k}+\sigma_{m 1} v_{1 k}, \\
& y_{2 k}=x_{2 k}+\dot{x}_{s k}+\sigma_{m 2} v_{2 k} .
\end{aligned}
$$

In the numerical work we generate the synthetic measurement by assuming $m=1.0 \mathrm{~kg}$, coefficient of viscous damping $\eta=0.04$, undamped natural frequency $\omega=4 \pi \mathrm{rad} / \mathrm{s}$, step size $h=(2 \pi / 80 \omega) \mathrm{s}, \sigma=0.0072 \mathrm{~N}$, $\sigma_{m 1}=1.1434 \times 10^{-4} \mathrm{~m}$ and $\sigma_{m 2}=1.5 \times 10^{-3} \mathrm{~m} / \mathrm{s}, \omega_{0}=2 \pi \mathrm{rad} / \mathrm{s}, n=5$, and amplitude of support displacement is $0.001 \mathrm{~m}$. The noise present in the measurement of displacement and velocity here are taken to be about $5 \%$ of the maximum value of the respective system states in the absence of noise.

We first consider the problem of state estimation from noisy measurements. It may be noted that this problem is amenable for an exact solution using the Kalman filter and also could be solved using the SIS particle filter. The two solutions are expected to show good mutual agreement. Indeed this is observed in Fig. 6 in which the Kalman filter estimate on the expected states is observed to compare well with similar results from a SIS filter with 500 particles. It was also verified in the numerical work that the variance of the estimate using particle filter approached the exact Kalman filter values as number of particles used in the simulation increased. This preliminary verification ensures that the codes developed for implementing the SIS filter are performing correctly.

The problem of determining the system stiffness $k_{o}$ is considered next. Measurement data on displacement and velocity are generated with a reference value of $k_{o}=157.91 \mathrm{~N} / \mathrm{m}$. These data are seeded with measurement noise of standard deviations $\sigma_{m 1}=1.1434 \times 10^{-4} \mathrm{~m}$ and $\sigma_{m 2}=1.5 \times 10^{-3} \mathrm{~m} / \mathrm{s}$. The evolution of weights on the stiffness parameter is shown in Fig. 7(a)-(e) for five cycles of iteration. In the first cycle of iteration $k_{o}$ is postulated to be a random variable with four discrete states $k_{o}=100,150,200$, and $250 \mathrm{~N} / \mathrm{m}$ with equal probability. The evolution of weights under this hypothesis is shown in Fig. 7a. As may be observed, the weights corresponding to $k_{o}=150 \mathrm{~N} / \mathrm{m}$ possess the highest weight that tends to unity with all others weights going to zero. Based on this, in the subsequent cycle of filtering, the range of $k_{o}$ is reduced and is now postulated to be a random variable with states $k_{o}=125,150,175$ and $200 \mathrm{~N} / \mathrm{m}$. Fig. 7(b) shows the growth of weight corresponding to the state $k_{o}=150 \mathrm{~N} / \mathrm{m}$ to a value of unity. In the subsequent cycles, the range of $k_{o}$ is further reduced and, at the end of fifth cycle, $k_{o}=157.5 \mathrm{~N} / \mathrm{m}$ is observed to possesses the highest weight. Figs. 8(a) and (b) show, respectively, the evolution of expected value of $k_{o}$ and the variance during the fifth cycle of filtering. As may be observed, the algorithm estimates $k_{o}=156.80 \mathrm{~N} / \mathrm{m}$, which compares well with the reference value of $157.91 \mathrm{~N} / \mathrm{m}$. Fig. 9 shows the estimates of the displacement and velocity states at the end of the fifth cycle of iteration. The estimated values are observed to be close to the measured values.

\subsection{Example 2: Nonlinear sdof Duffing's oscillator}

Fig. 3 shows the system under consideration. The governing process equations are written in the standard form as

$$
\begin{aligned}
\mathrm{d} x_{1} & =x_{2} \mathrm{~d} t, \\
\mathrm{~d} x_{2} & =\left(-\frac{c}{m} x_{2}-\frac{k_{o}}{m} x_{1}-\frac{\alpha}{m} x_{1}^{3}+\frac{P}{m} \cos \lambda t\right) \mathrm{d} t+\sigma \mathrm{d} B(t), \\
x_{1}(0) & =0 ; x_{2}(0)=0 .
\end{aligned}
$$




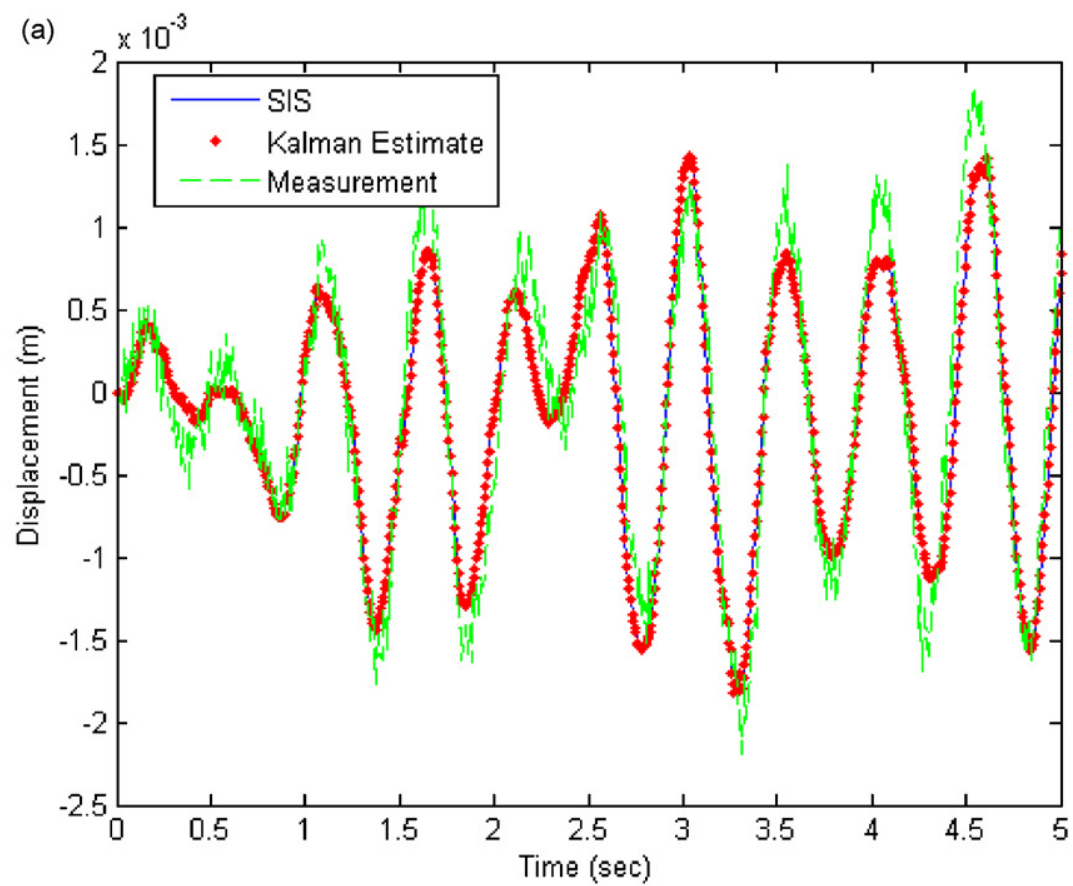

(b)

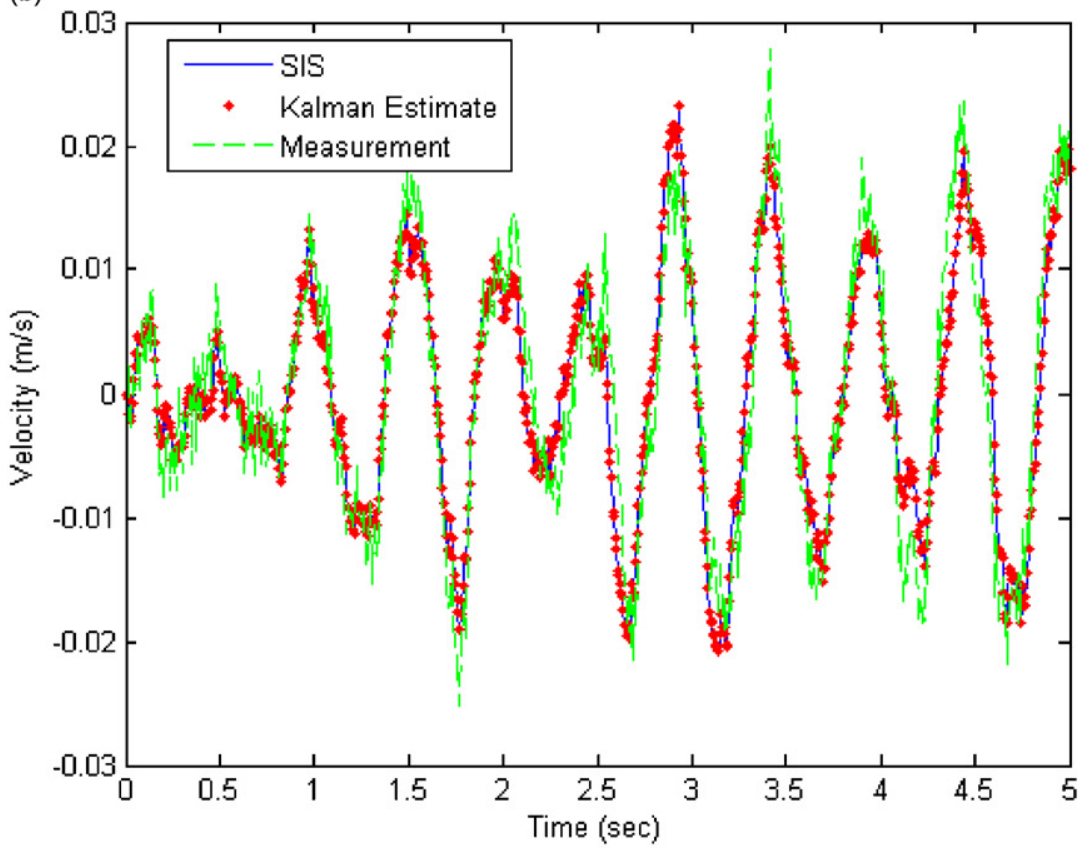

Fig. 6. Example 1: Estimation of states of sdof system using Kalman filter and Particle filter.

The discretized set of equations are obtained as

$$
\begin{aligned}
x_{1(k+1)}= & x_{1 k}+x_{2 k} h-\left(x_{1 k} k_{o}+x_{1 k}^{3} \alpha+c x_{2 k}-P \cos \lambda t_{k}\right) \frac{h^{2}}{2 m}+\frac{\sigma}{m} I_{10} \\
x_{2(k+1)}= & x_{2 k}-\left(x_{1 k} k_{o}+x_{1 k}^{3} \alpha+c x_{2 k}\right) \frac{h}{m}-\left(x_{2 k} k_{o} m+3 x_{2 k} x_{1 k}^{2}-c k_{o} x_{1 k}-c \alpha x_{1 k}^{3}-c^{2} x_{2 k}+c P \cos \lambda t_{k}\right) \\
& \times \frac{h^{2}}{2 m^{2}}+\frac{P}{\lambda}\left[\sin \lambda t_{k+1}-\sin \lambda t_{k}\right]-\frac{c}{m^{2}} \sigma I_{10}+\frac{\sigma}{m} I_{1} .
\end{aligned}
$$


(a)

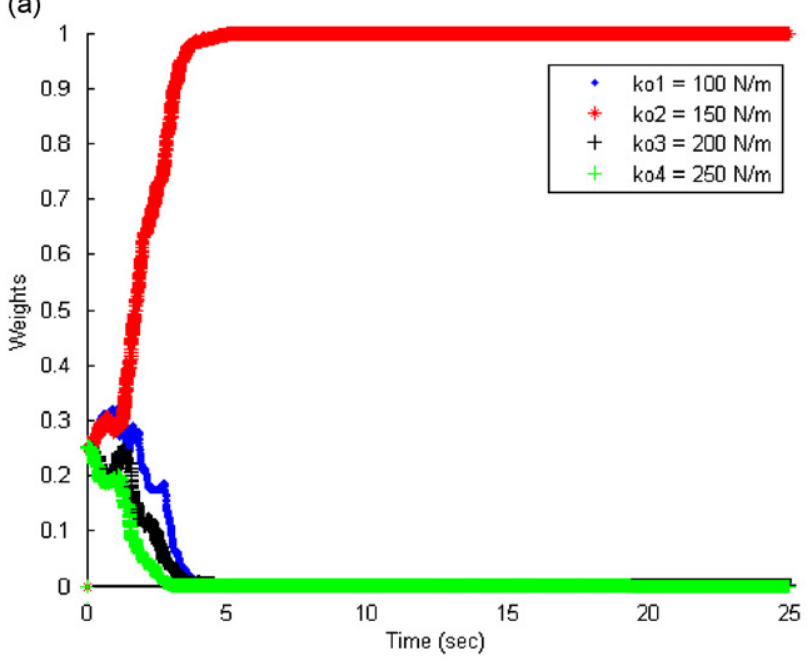

(c)

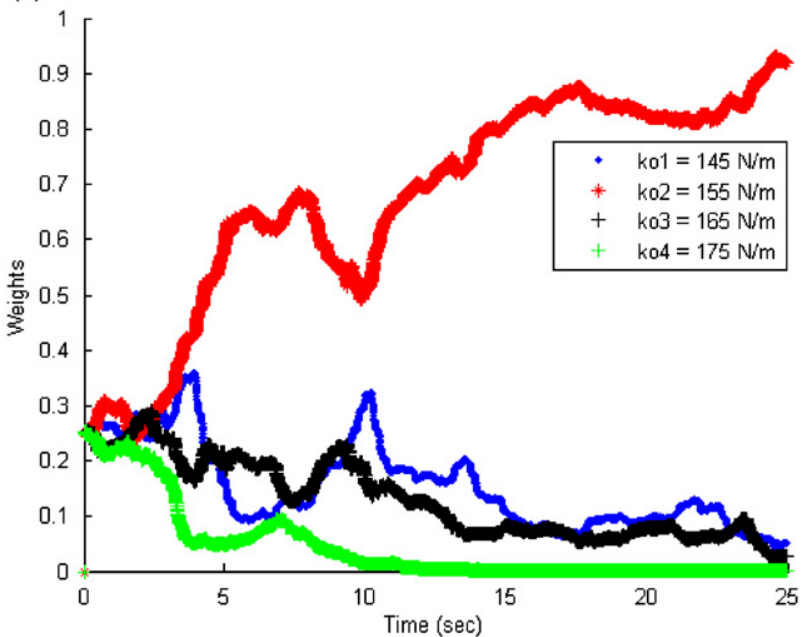

(d)

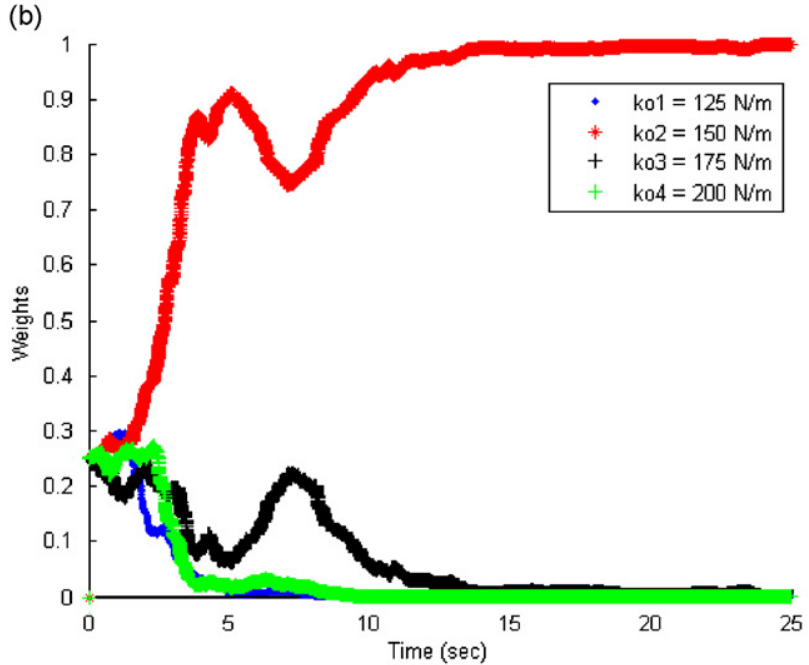

(e)

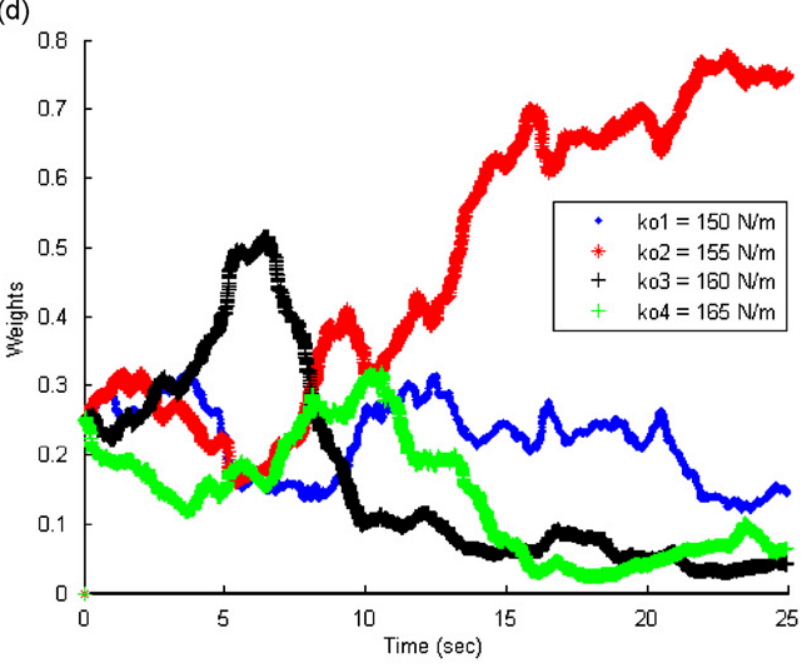

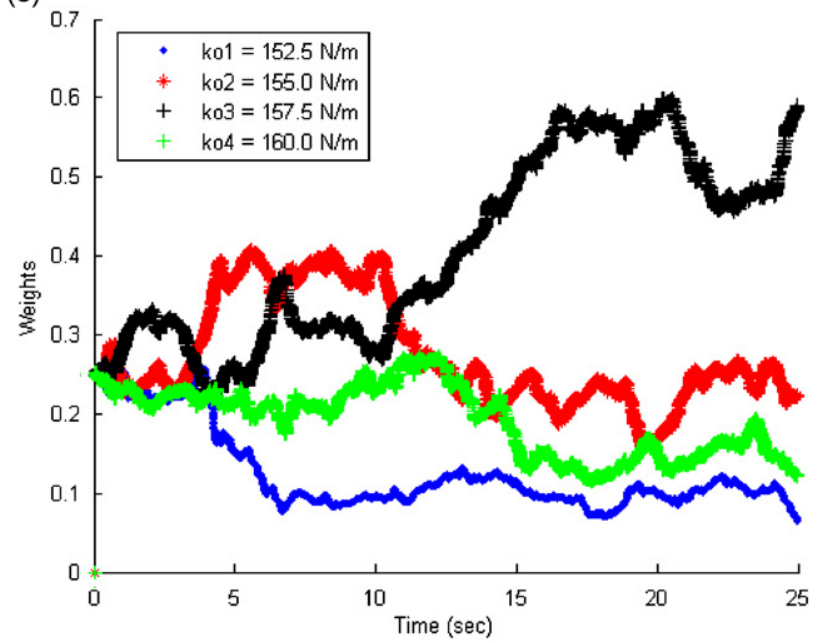

Fig. 7. Example 1: Estimation of system stiffness parameter $k_{o}$ using bank of SIS filters: (a)-(e) evolution of weights on the stiffness parameter for the first five cycles; the legends show the states of $k_{o}$ in $\mathrm{N} / \mathrm{m}$; the reference value of $k_{o}$ is $157.91 \mathrm{~N} / \mathrm{m}$. (a) cycle 1 , (b) cycle 2 , (c) cycle 3, (d) cycle 4, (e) cycle 5 . 
(a)

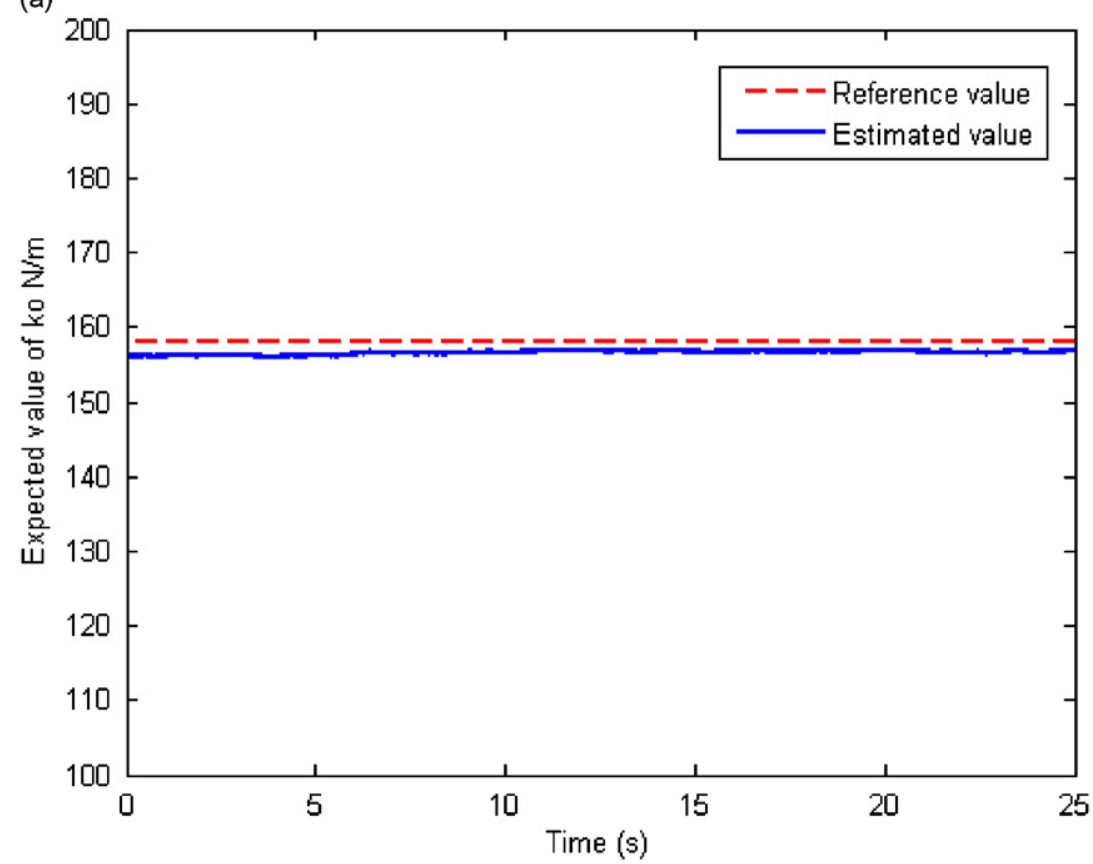

(b)

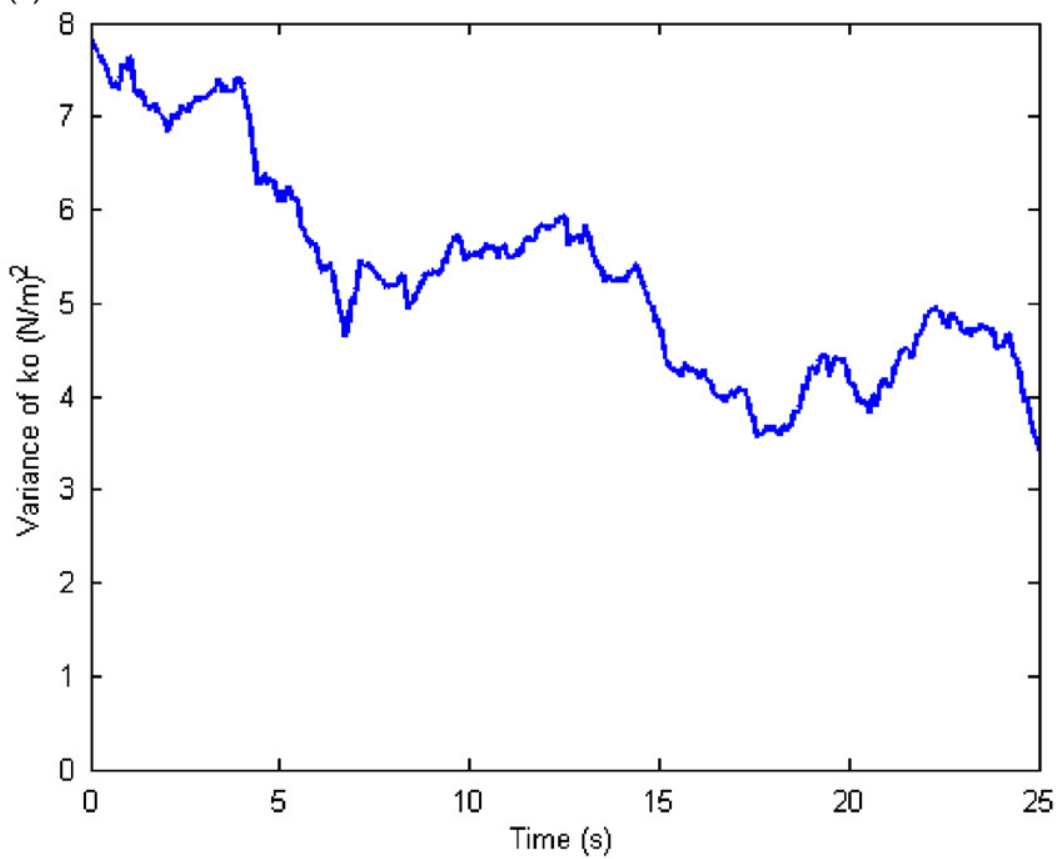

Fig. 8. Example 1: Estimation of system stiffness parameter $k_{o}$ using bank of SIS filters; (a) expected value of $k_{o}$; (b) variance of stiffness; the reference value of $k_{o}$ is $157.91 \mathrm{~N} / \mathrm{m}$.

The quantities $I_{1}$ and $I_{10}$ are as defined in Eq. (32). The measurement is assumed to be made on the velocity response and accordingly, we get

$$
y_{k}=x_{2 k}+\sigma_{m} v_{k} .
$$

Here the process equation is nonlinear and the problem is amenable for solution via the bank of SIS filter approach. In the numerical work we take $m=1.0 \mathrm{~kg}, \eta=0.04, k_{o}=16 \pi^{2} \mathrm{~m} \mathrm{~N} / \mathrm{m}, \omega=4 \pi \mathrm{rad} / \mathrm{s}, P=10.106 \mathrm{~N}$, 


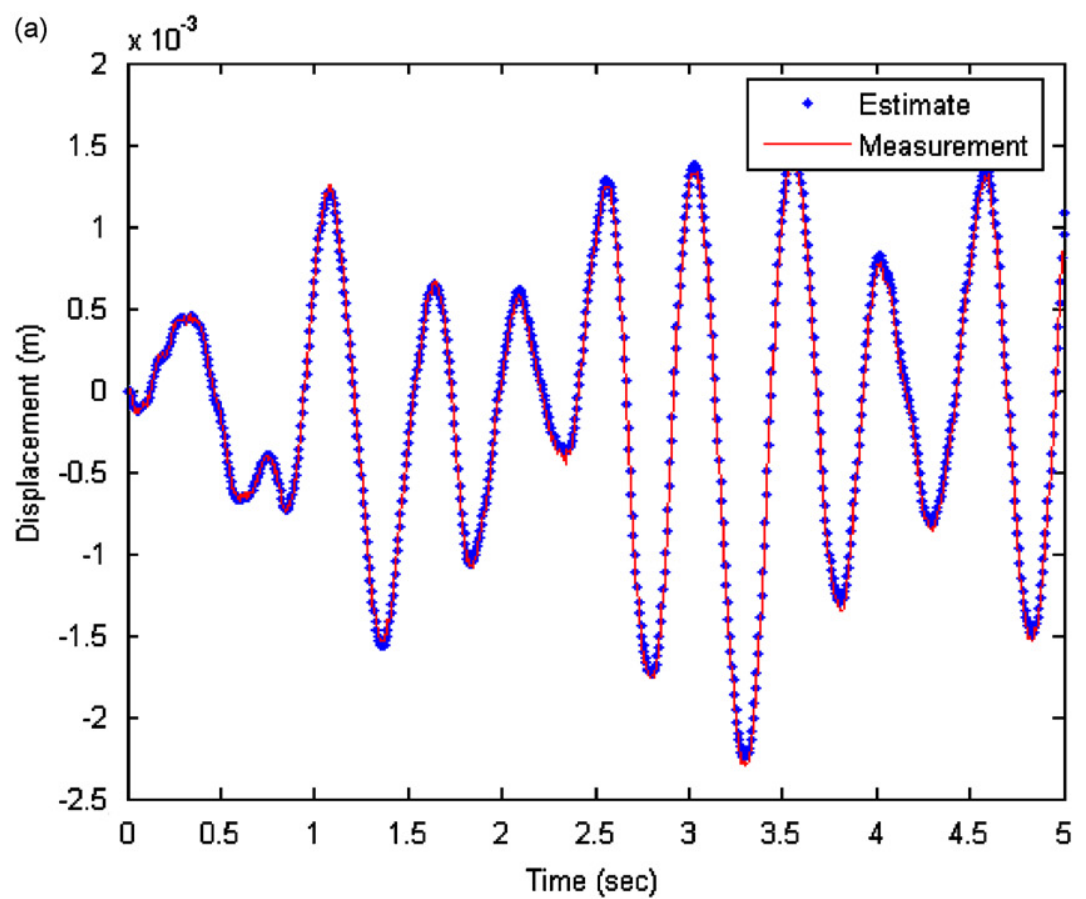

(b)

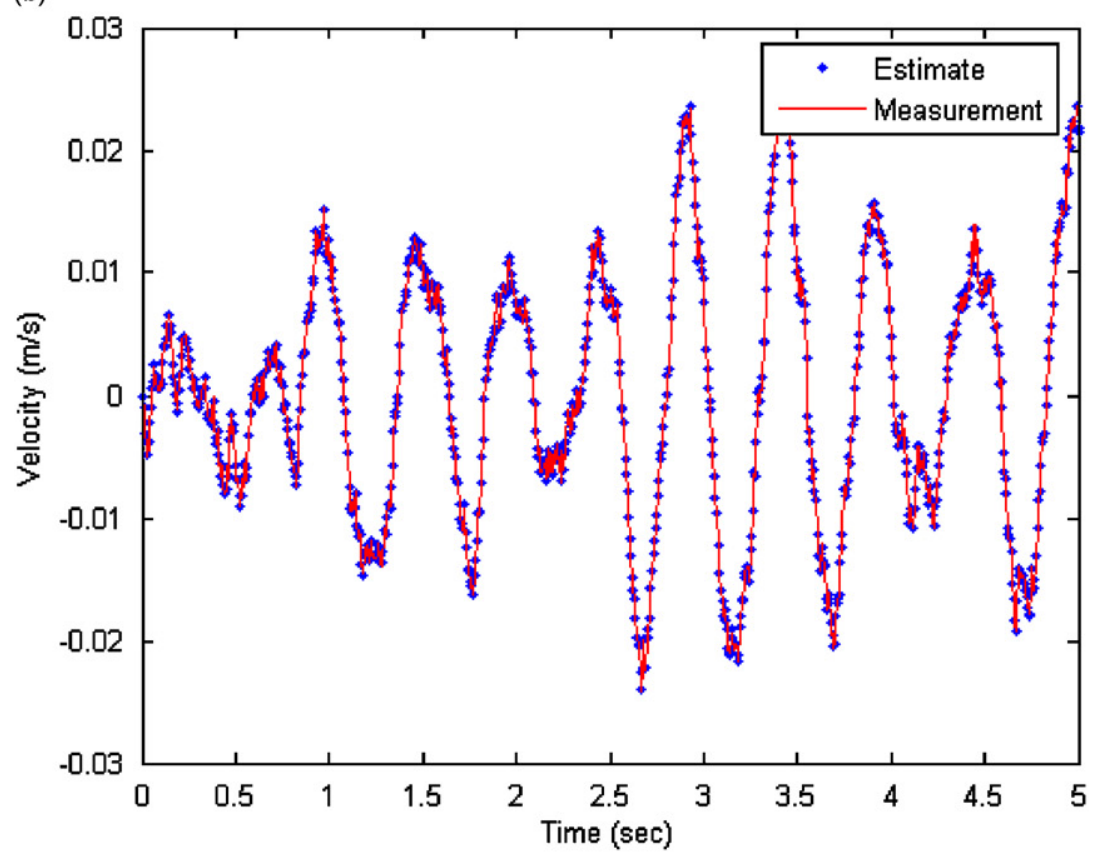

Fig. 9. Example 1: Estimation of displacement and velocity states at the end of the fifth cycle of iteration using bank of SIS filters.

$\sigma=P / 20, h=(2 \pi / 80 \lambda) \mathrm{s}$, and $\lambda=0.8 \omega \mathrm{rad} / \mathrm{s}$. We consider the measurement noise having zero mean and standard deviation, $\sigma_{m}=0.0279 \mathrm{~m} / \mathrm{s}$. Synthetic data is generated with an assumed reference value of $\alpha=3.45 \times 10^{5} \mathrm{~N} / \mathrm{m}^{3}$. The problem on hand consists of determining the nonlinearity parameter $\alpha$. The results using bank of SIS filter banks, with each bank consisting of 500 particles, are shown in Figs. 10-13. For the system parameters chosen, it is clear from the phase-plane plot shown in Fig. 10 that the system behavior is indeed influenced by the nonlinearity parameter $\alpha$. It may be noted that this plot has been produced in the 


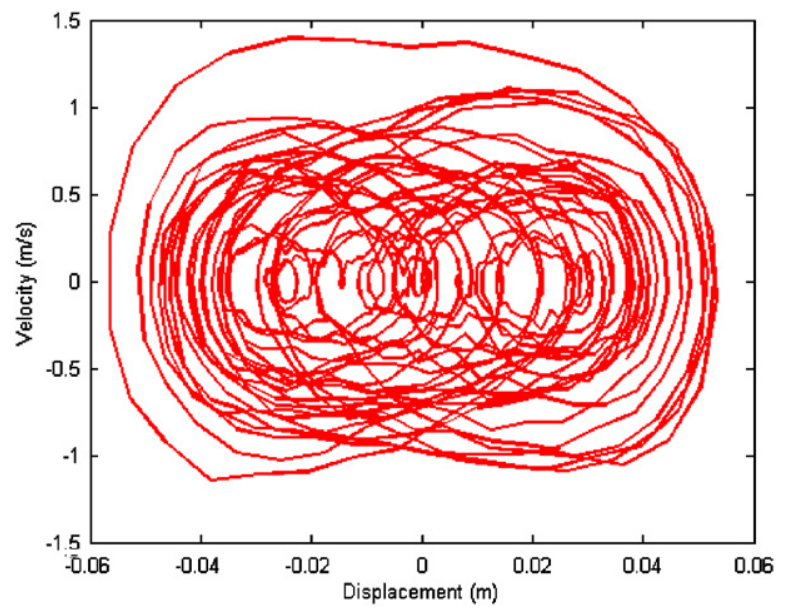

Fig. 10. Example 2: Phase-plane plot of the Duffing's oscillator showing nonlinearity with the assumed value of $\alpha$ (a nonlinear stiffness parameter).

absence of noise terms. The evolution of weights associated with the states of $\alpha$ is shown in Fig. 11(a)-(g). As the cycles of filtering proceeds the initial range of $\alpha$ from $2 \times 10^{5}-5 \times 10^{5} \mathrm{~N} / \mathrm{m}^{3}$ narrows down to the range $3.250 \times 10^{5}-3.325 \times 10^{5} \mathrm{~N} / \mathrm{m}^{3}$, which is near the reference value of $\alpha=3.45 \times 10^{5} \mathrm{~N} / \mathrm{m}^{3}$. Fig. 12 shows the evolution of expected value of $\alpha$ and the variance of $\alpha$ during the seventh cycle of filtering. These moments at the end of $10 \mathrm{~s}$ are, respectively, $3.28 \times 10^{5} \mathrm{~N} / \mathrm{m}^{3}$ and $0\left(\mathrm{~N} / \mathrm{m}^{3}\right)^{2}$. The reason for variance being zero is that the weights associated with one of the state $\alpha$ go to one, while all other weights go to zero. The estimate of the expected velocity of the states of the system is shown in Fig. 13. The evolution of this estimate is observed to closely follow the trajectory of the measured velocity.

\subsection{Example 3: Nonlinear sdof system with bilinear stiffness characteristics}

Fig. 4 shows a spring-dash pot supported mass element that is suspended from a cable. Since cable does not carry compressive forces, the system possesses differing stiffnesses during upward and downward displacements. The governing equation of motion for this system can be given by

$$
\begin{aligned}
\mathrm{d} x_{1} & =x_{2} \mathrm{~d} t, \\
\mathrm{~d} x_{2} & =\left(-\frac{c}{m} x_{2}-\frac{k_{o}}{m} x_{1}-\frac{k_{s}}{m} x_{1} U\left[x_{1}+x_{s}\right]-\frac{f(t)}{m}\right) \mathrm{d} t+\sigma \mathrm{d} B(t), \\
x_{1}(0) & =0 ; x_{2}(0)=0,
\end{aligned}
$$

with $f(t)=m \ddot{x}_{s}(t)+k_{s} x_{s} U\left[x_{1}+x_{s}\right]$. Here $U[\cdot]$ is the Heaveside step function. The discretized set of equations of motion are obtained as

$$
\begin{aligned}
x_{1(k+1)}= & x_{1 k}+x_{2 k} h+\left\{-\frac{k_{o}}{m} x_{1 k}-\frac{k_{s}}{m} x_{1 k} U\left[x_{1 k}+x_{s k}\right]-\frac{c}{m} x_{2 k}-\ddot{x}_{s k}-\frac{k_{s}}{m} x_{s k} U\left[x_{1 k}+x_{s k}\right]\right\} \frac{h^{2}}{2}+\frac{\sigma}{m} I_{10}, \\
x_{2(k+1)=} & x_{2 k}+x_{2 k} h-\left\{\frac{k_{o}}{m} x_{1 k}-\frac{k_{s}}{m} x_{1 k} U\left[x_{1 k}+x_{s k}\right]+\frac{c}{m} x_{2 k}+v_{s k}+\frac{k_{s}}{m} d_{s k} U\left[x_{1 k}+x_{s k}\right]\right\} h \\
& +x_{2 k}\left(-\frac{k_{s}}{m} \delta\left(x_{1 k}+x_{s k}\right) x_{1 k}-\frac{k_{o}}{m}-\frac{k_{s}}{m} U\left[x_{1 k}+x_{s k}\right]-\frac{k_{s}}{m} x_{s k} \delta\left(x_{1 k}+x_{s k}\right)\right) \frac{h^{2}}{2} \\
& -\left\{-\frac{k_{o}}{m} x_{1 k}-\frac{k_{s}}{m} x_{1 k} U\left[x_{1 k}+x_{s k}\right]-\frac{c}{m} x_{2 k}-\ddot{x}_{s k}-\frac{k_{s}}{m} x_{s k} U\left[x_{1 k}+x_{s k}\right]\right\} \frac{1}{m c} \frac{h^{2}}{2}-\frac{c}{m^{2}} \sigma I_{10}+\frac{\sigma}{m} I_{1},
\end{aligned}
$$


with

$$
d_{s k}=\int_{t_{k}}^{t_{k+1}} x_{s k} \mathrm{~d} t=\left[x_{s(k+1)}+x_{s k}\right] \frac{h}{2} .
$$

In Eq. (38), $\delta(\cdot)$ is the Dirac delta function and $I_{1}$ and $I_{10}$ are as defined in Eq. (48). The presence of Dirac delta function on the right-hand side of above equation is indicative of the fact that the drift term in Eq. (37) has a Heaveside step function which is non-differentiable at $x_{1}+x_{s}=0$. Even though the event $x_{1}+x_{s}=0$, has a measure zero of occurrence, its occurrence is ideally required to be detected when the solutions are sought in the strong form. The treatment of stochastic differential equations in which such events are to be detected during the solution process is still an open area of research and we have not addressed this issue in our work. Therefore, in the numerical work, we either altogether drop these terms or replace the Dirac delta function by a Gaussian pdf with zero mean and arbitrarily small standard deviation. It was observed in the numerical studies that the estimates of the states and the estimate of parameter were not notably influenced by this choice.

(a)

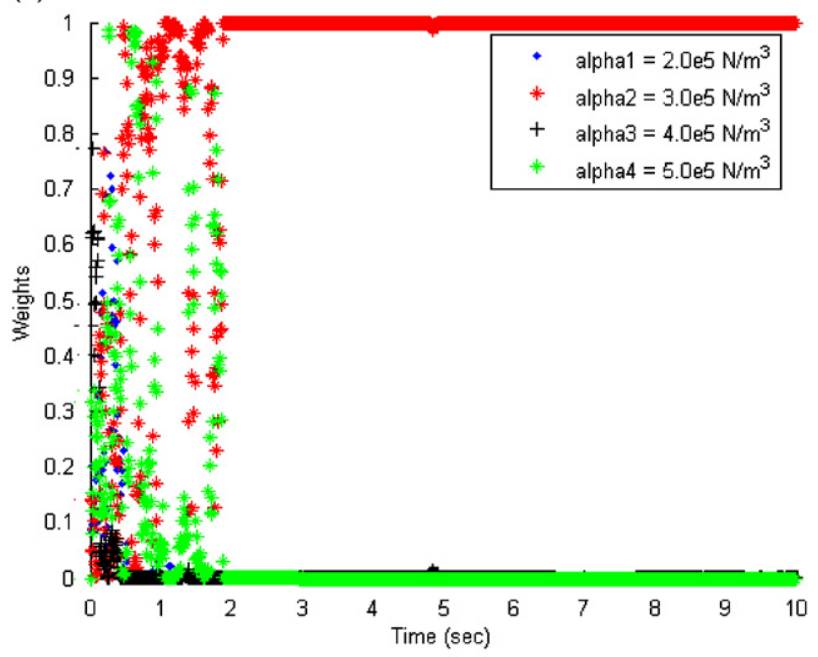

(b)

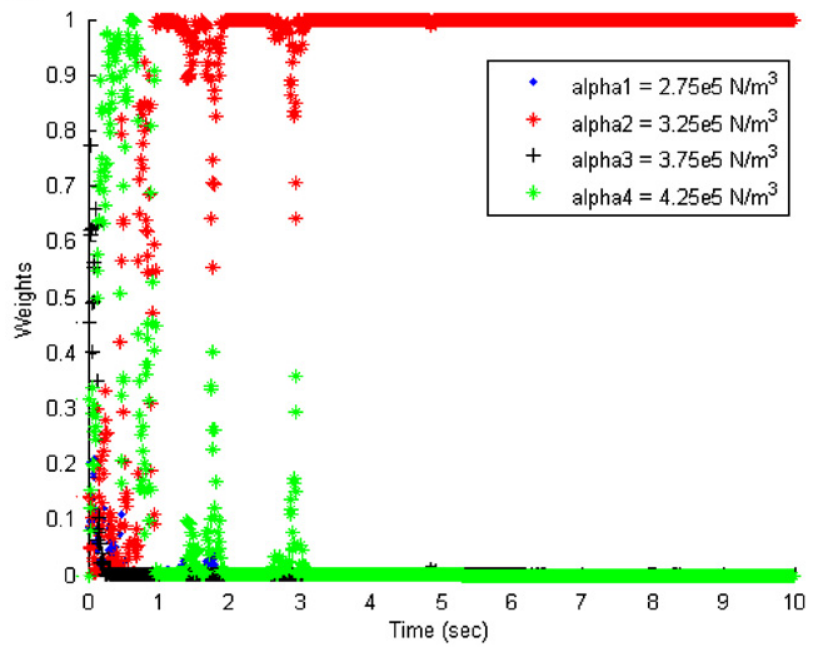

(c)

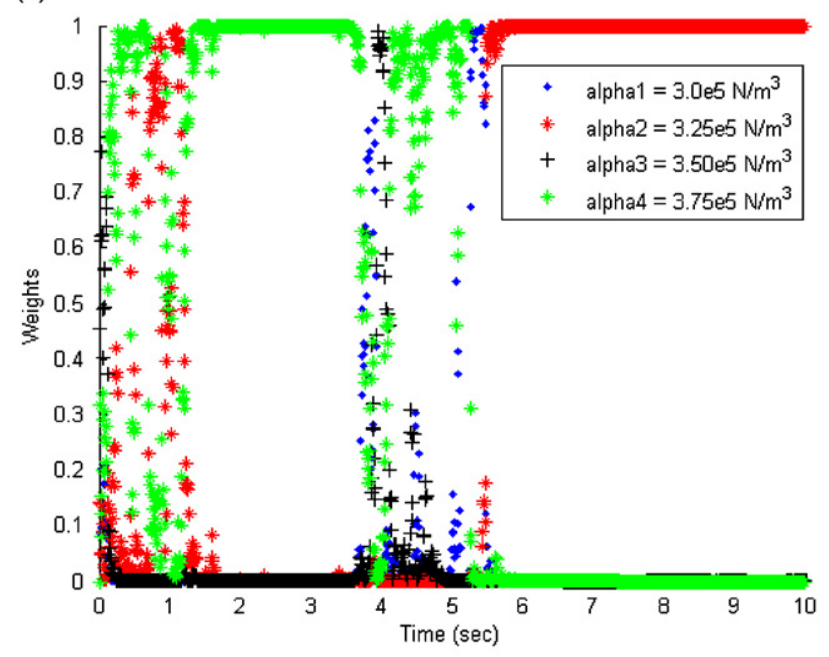

(d)

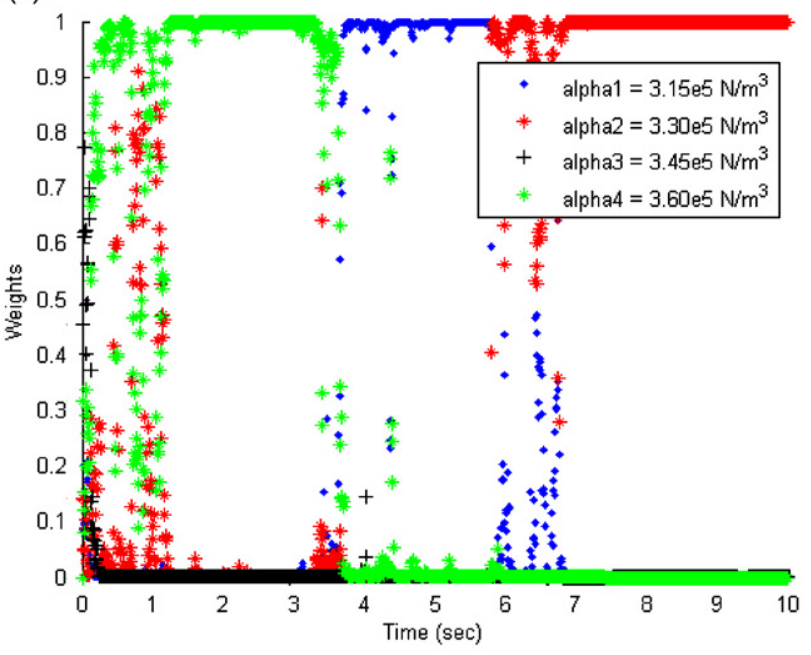

Fig. 11. Example 2: Estimation of nonlinear stiffness parameter $\alpha$ using bank of SIS filters: (a)-(g) evolution of weights on $\alpha$ for the first seven cycles. Legends show the states of $\alpha$ in $\mathrm{N} / \mathrm{m}^{3}$. Reference value of $\alpha$ is $3.45 \times 10^{5} \mathrm{~N} / \mathrm{m}^{3}$. (a) cycle 1 , (b) cycle 2, (c) cycle 3, (d) cycle 4 , (e) cycle 5, (f) cycle 6, (g) cycle 7. 
(e)

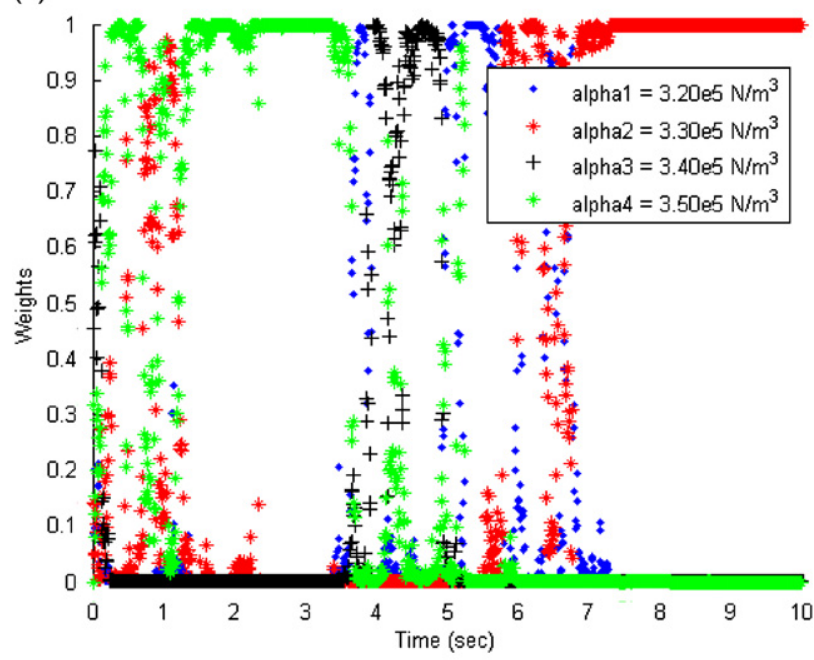

(f)

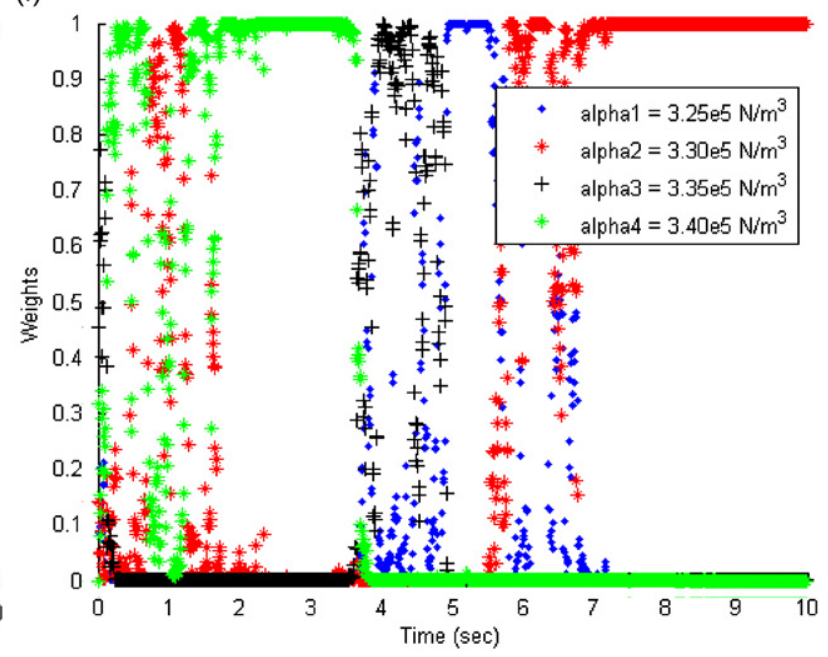

(g)

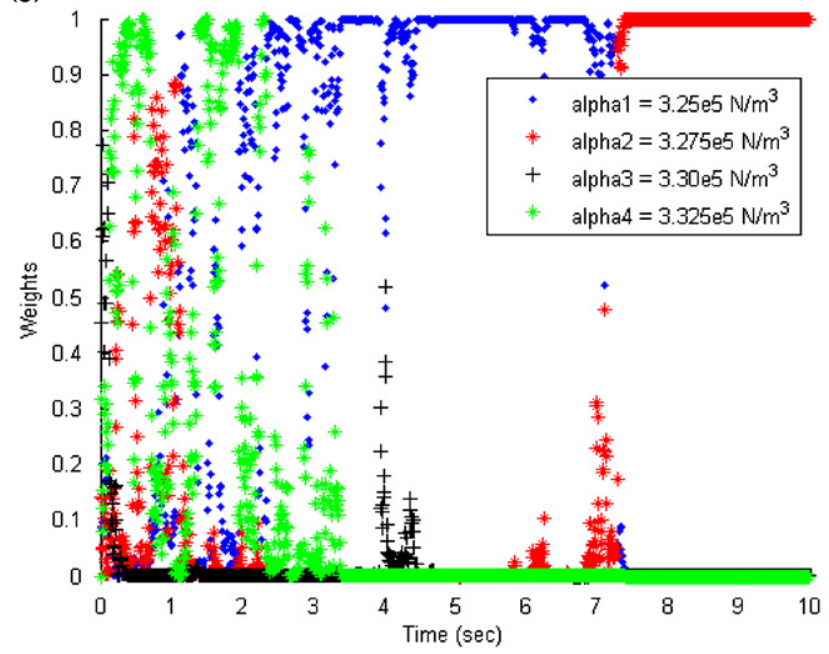

Fig. 11. (Continued)

The measurement here is made on the velocity response, and, accordingly the measurement equation reads

$$
y_{k}=x_{2 k}+\sigma_{m} v_{k} \text {. }
$$

Thus, in this example, the process equation is nonlinear, the measurement equation is linear in the state variables, and the noise processes are additive. In the numerical work we take $m=1.0 \mathrm{~kg}, \eta=0.04$, $k_{o}=16 \pi^{2} m \mathrm{~N} / \mathrm{m}, \omega=4 \pi \mathrm{rad} / \mathrm{s}, \sigma=0.0051 \mathrm{~N}, h=(2 \pi / 80 \omega) \mathrm{s}$. We consider the measurement noise having zero mean and standard deviation $\sigma_{m}=0.0013 \mathrm{~m} / \mathrm{s}$. The applied support displacement is taken to be the same as considered in Example 1 (Eq. (29)). Initially the system is taken to be at rest. Synthetic data are generated with a reference value of $k_{s}=245.0 \mathrm{~N} / \mathrm{m}$. For implementing the bank of SIS filter approach for the identification of parameter, we take $N=500$ samples. Figs. 14-16 shows the result on estimation on cable stiffness and system velocity. From Figs. 14 and 15, it is evident that the algorithm is successfully predicting the cable stiffness. Thus, we get the estimate for the expected value of cable stiffness to be about $244.0 \mathrm{~N} / \mathrm{m}$ which compares very well with the reference value of $k_{s}=245.0 \mathrm{~N} / \mathrm{m}$. Similarly, the estimated expected value of system velocity shows good comparison with the measured velocity in Fig. 16. 

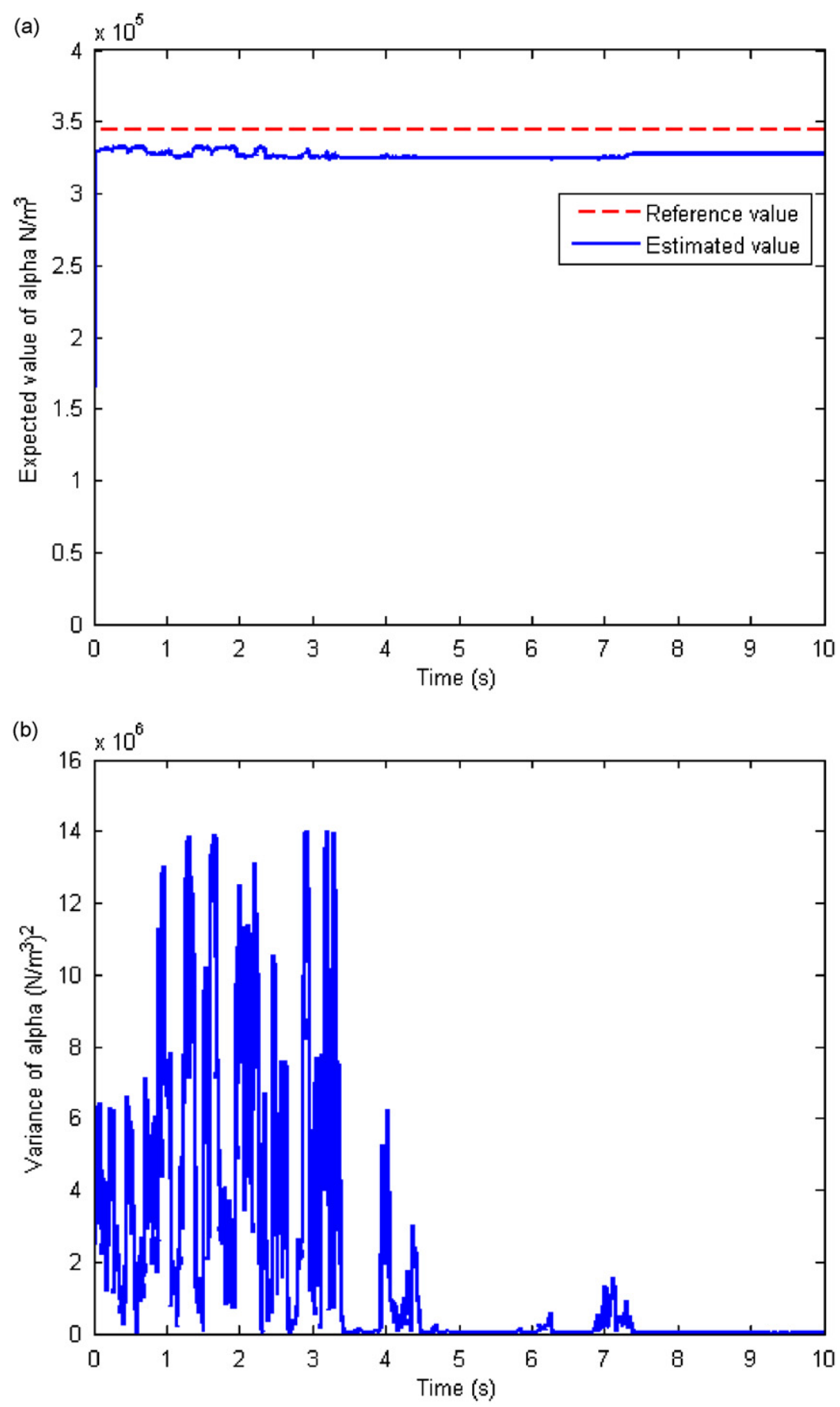

Fig. 12. Example 2: Estimation of nonlinearity parameter $\alpha$ using bank of SIS filters: (a) expected value of $\alpha$, (b) variance of $\alpha$; reference value of $\alpha$ is $3.45 \times 10^{5} \mathrm{~N} / \mathrm{m}^{3}$.

\subsection{Example 4: An Euler-Bernoulli beam suspended on cables}

Here we consider an Euler-Bernoulli beam which is supported by a set of cables and which is subjected to support excitations as shown in Fig. 5. Since the cables cannot carry compressive forces, the beam-cable 


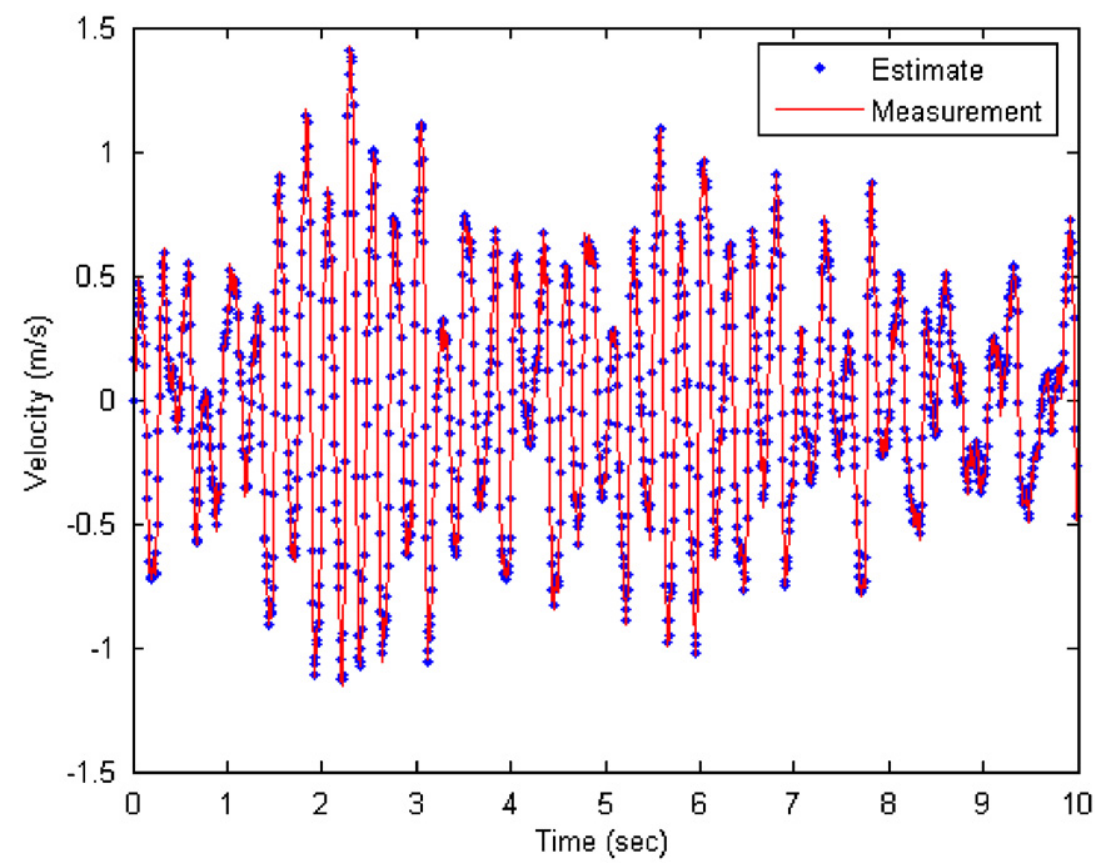

Fig. 13. Example 2: Estimation of the velocity state of the system using bank of SIS filters.

system would possess nonlinear stiffness characteristics. The motivation for selecting this example is that the beam-cable system considered here can easily be studied experimentally. The governing equations of motion can be formulated as

$$
\begin{aligned}
& E I y^{i v}(x, t)+m_{b} \ddot{y}(x, t)+c \dot{y}(x, t)+\sum_{i=1}^{3} m_{i} \delta\left(x-\xi_{i}\right) \ddot{y}(x, t)+\sum_{i=1}^{3} k_{s i} y(x, t) \delta\left(x-\xi_{i}\right) U[y(x, t)]=0 \\
& y(0, t)=x_{s}(t) ; \quad K_{r} y^{\prime}(0, t)-E I y^{\prime \prime}(0, t)=0, \\
& E I y^{\prime \prime}(L, t)=0 ; \quad E I y^{\prime \prime \prime}(L, t)=0, \\
& y(x, 0)=0 ; \quad \dot{y}(x, 0)=0 .
\end{aligned}
$$

A prime here denotes differentiation with respect to $x$ and a dot, as before, the derivative with respect to time $t$. Furthermore, $E I, m_{b}$, and $c$, are, respectively, the flexural rigidity, beam mass per unit length and coefficient of viscously damping; $K_{r}$ is the stiffness of rotary spring at $x=0$ that represents the possible lack of adequate fixity at the support; $\left\{m_{i}\right\}_{i=1}^{3}$ are the concentrated masses that represent the fixtures at the cable attachment points, and $\left\{k_{s i}\right\}_{i=1}^{3}$ represent the cable stiffness in tension. The above equation is nonlinear in nature and the system receives external excitations through the boundary condition at $x=0$. This would mean that the equation has time-dependent boundary conditions. We introduce a new dependent variable $y(x$, $t)=z(x, t)+x_{s}(t)$ so that the governing equation can be re-written as

$$
\begin{aligned}
& E I z^{i v}(x, t)+m_{b} \ddot{z}(x, t)+c \dot{z}(x, t)+\sum_{i=1}^{3} m_{i} \delta\left(x-\xi_{i}\right) \ddot{z}(x, t) \\
& +\sum_{i=1}^{3} k_{s i} z(x, t) \delta\left(x-\xi_{i}\right) U\left[z(x, t)+x_{s}(t)\right]=-m_{b} \ddot{x}_{s}-c \dot{x}_{s}-\sum_{i=1}^{3} m_{i} \delta\left(x-\xi_{i}\right) \ddot{x}_{s}(x, t) \\
& +\sum_{i=1}^{3} k_{s i} x_{s}(t) \delta\left(x-\xi_{i}\right) U\left[z(x, t)+x_{s}(t)\right],
\end{aligned}
$$




$$
\begin{array}{ll}
z(0, t)=0, & K_{r} z^{\prime}(0, t)-E I z^{\prime \prime}(0, t)=0, \\
E I z^{\prime \prime}(l, t)=0, & E I z^{\prime \prime \prime}(l, t)=0, \\
z(x, 0)=-x_{s}(0), & \dot{z}(x, 0)=-\dot{x}_{s}(0) .
\end{array}
$$

It may be noted that the transformation of the dependent variable as above has ensured that the boundary conditions in the transformed equations of motion are independent of time and the effect of support motions appear on the right-hand side of the governing equation. We solve the above equation approximately using the method of weighted residuals by taking the solution to be of the form

$$
z(x, t)=\sum_{n=1}^{\infty} a_{n}(t) \phi_{n}(x)
$$

(a)

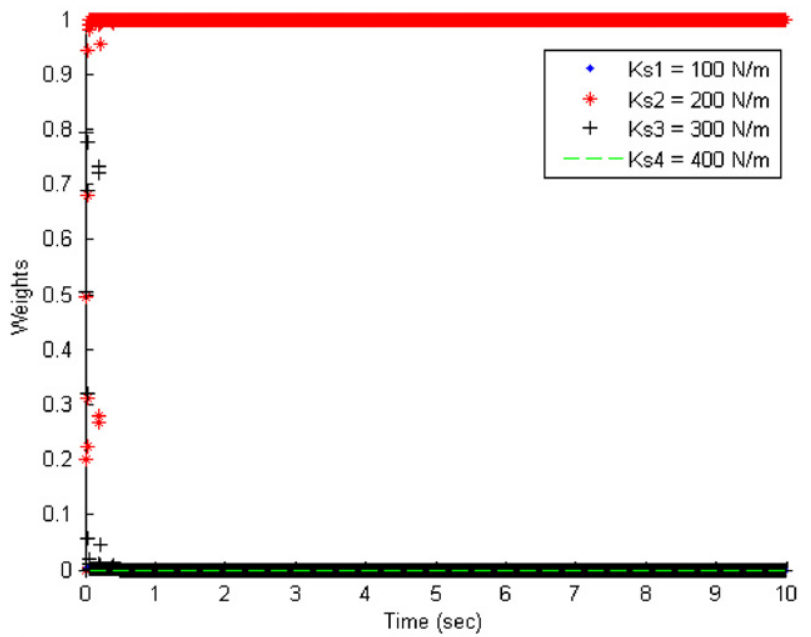

(b)

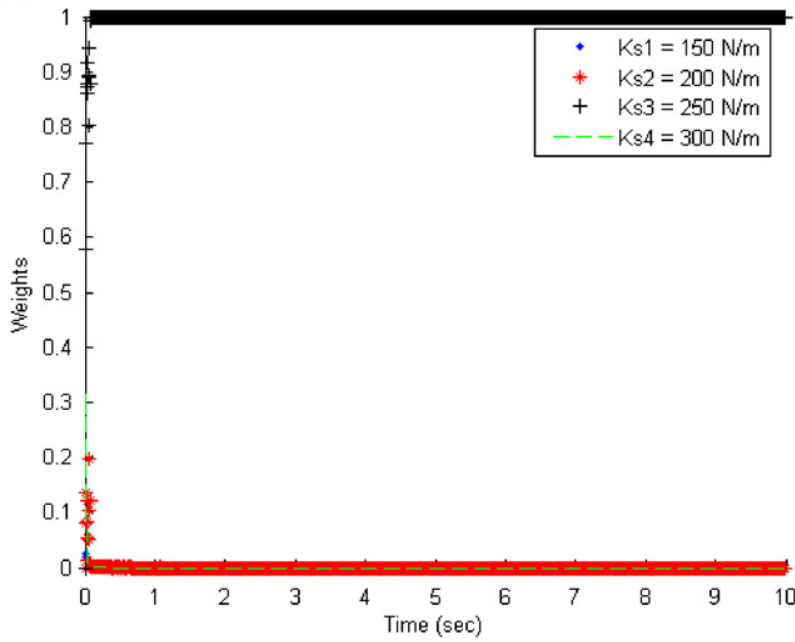

(c)

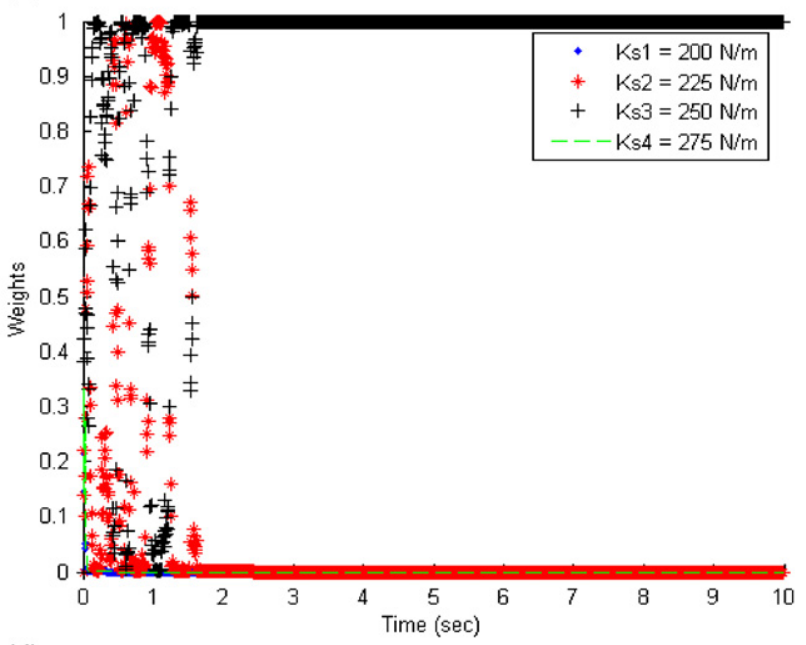

(d)

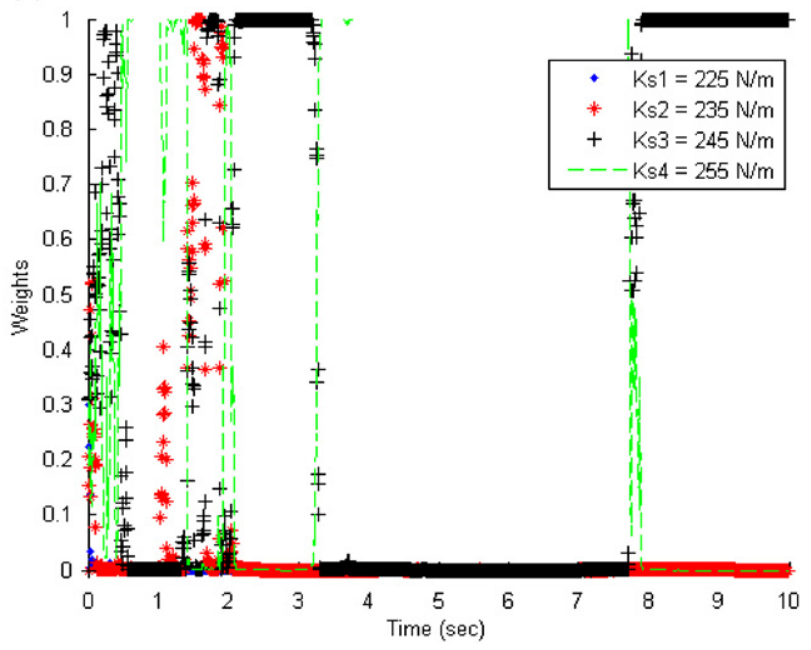

Fig. 14. Example 3: Estimation of cable stiffness parameter $k_{s}$ of the nonlinear sdof system with bilinear stiffness characteristics using bank of SIS filters: (a)-(g) evolution of weights on $k_{s}$ for the first seven cycles. Legends show the states of $k_{s}$ in N/m. Reference value of $k_{s}$ is $245 \mathrm{~N} / \mathrm{m}$. (a) cycle 1 , (b) cycle 2 , (c) cycle 3, (d) cycle 4, (e) cycle 5, (f) cycle 6 , (g) cycle 7. 
(e)

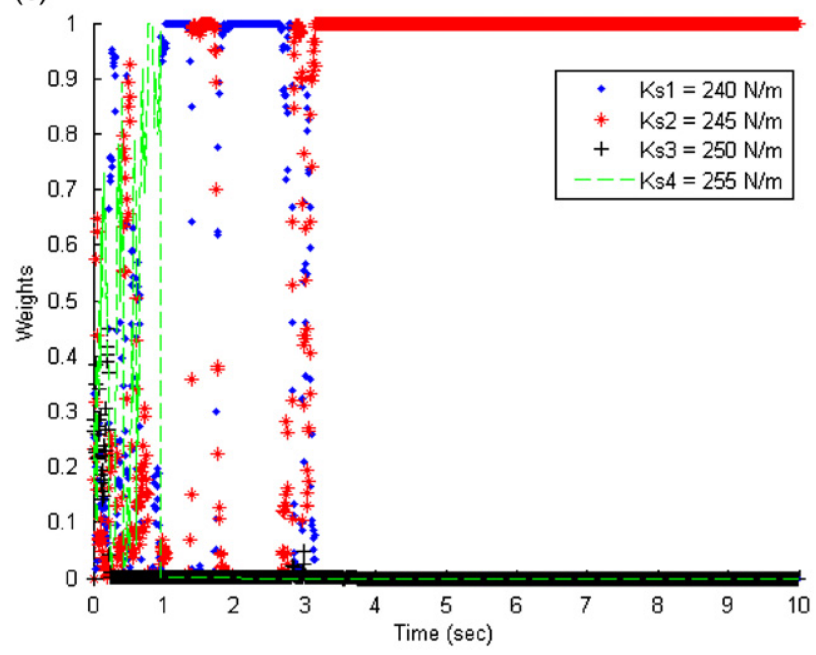

(f)

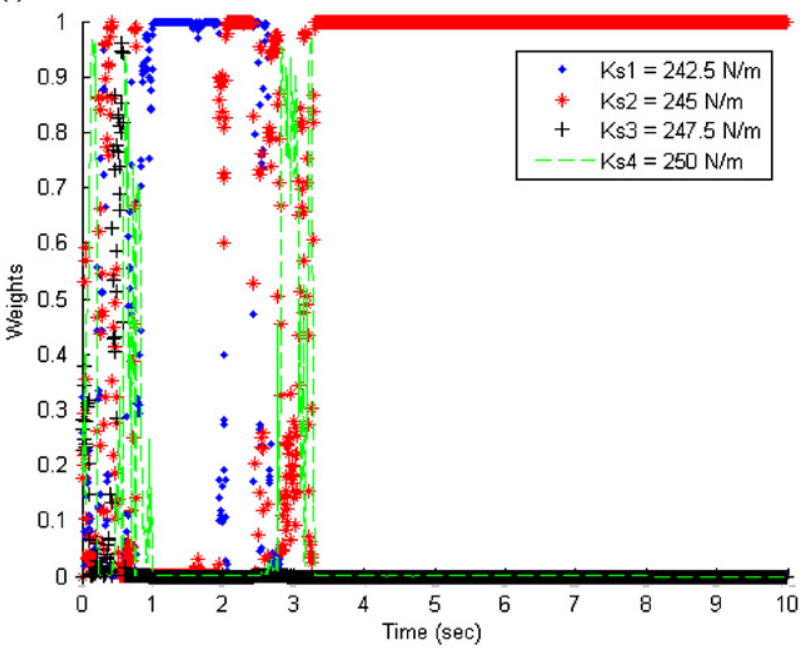

(g)

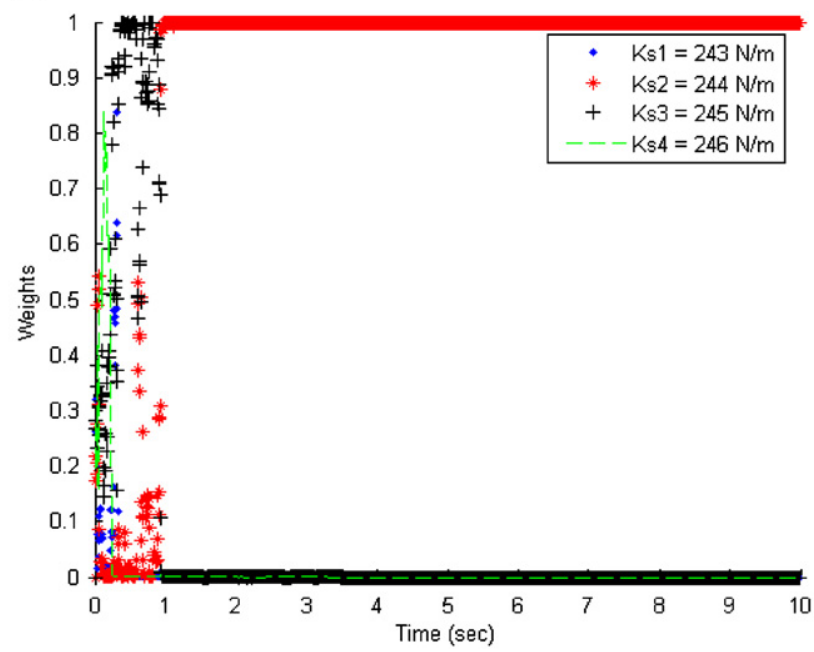

Fig. 14. (Continued)

Here $a_{n}(t)$ is the unknown $n$th generalized coordinate and $\phi_{n}(x)$ is the $n$th known trial function. The trial functions are determined by solving the linear free vibration problem

$$
E I z^{i v}(x, t)+m_{b} \ddot{z}(x, t)+\sum_{i=1}^{3} m_{i} \delta\left(x-\xi_{i}\right) \ddot{z}(x, t)=0
$$

using the Rayleigh-Ritz method. The trial functions $\left\{\phi_{n}(x)\right\}_{n=1}^{\infty}$ are selected to satisfy the following orthogonality conditions:

$$
\int_{0}^{l}\left[m_{b}+\sum_{i=1}^{3} m_{i} \delta\left(x-\xi_{i}\right)\right] \phi_{n}(x) \phi_{k}(x) \mathrm{d} x=\delta_{n k} \quad \text { and } \quad E I \phi_{n}^{i v}(x)=\omega_{n}^{2}\left[m_{b}+\sum_{i=1}^{3} m_{i} \delta\left(x-\xi_{i}\right)\right] \phi_{n}(x) .
$$




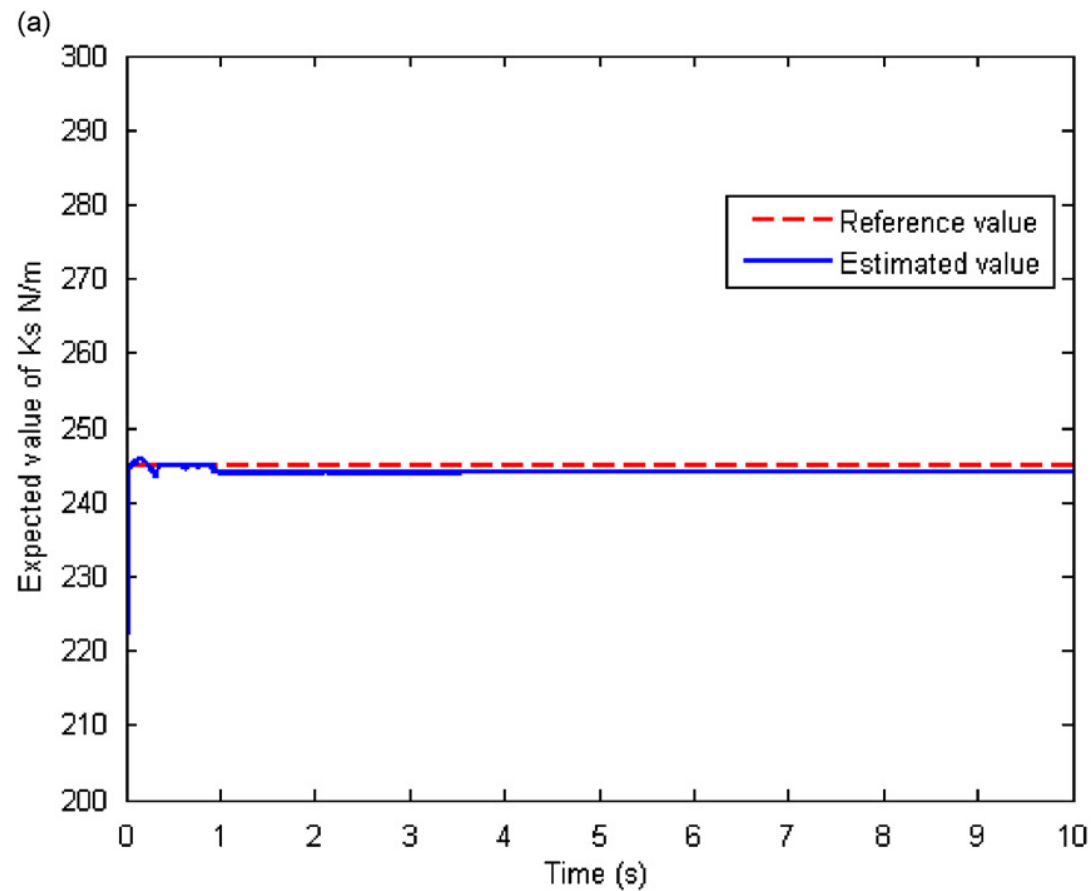

(b)

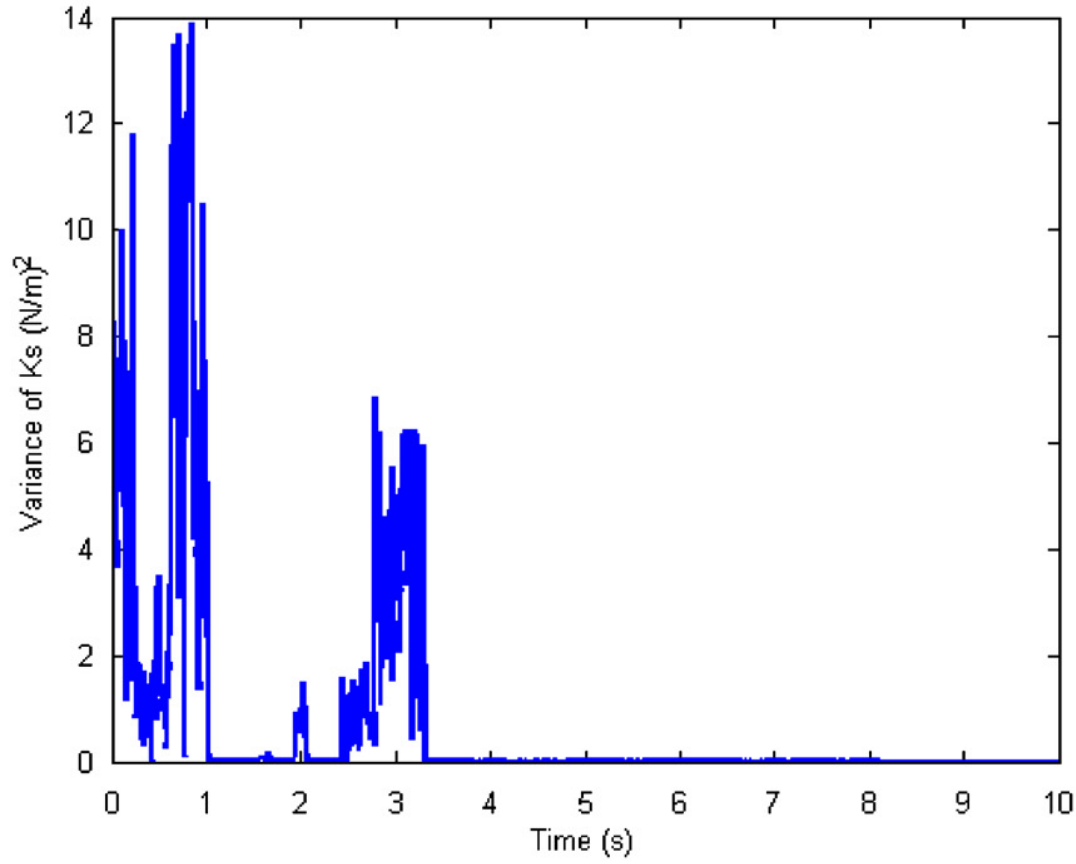

Fig. 15. Example 3: Estimation of cable stiffness parameter $k_{s}$ of the nonlinear sdof system with bilinear stiffness characteristics using bank of SIS filters: (a) expected value of $k_{s}$, (b) variance of $k_{s}$. Reference value of $k_{s}$ is $245 \mathrm{~N} / \mathrm{m}$.

Based on the method of weighted residuals, with $\phi_{n}(x)$ as the weight function, we get the equations for the generalized coordinate as

$$
\ddot{a}_{k}+2 \eta_{k} \omega_{k} \dot{a}_{k}+\omega_{k}^{2} a_{k}+\sum_{n=1}^{\infty} \sum_{i=1}^{3} k_{s i} a_{n}(t) \phi_{n}\left(\xi_{i}\right) \phi_{k}\left(\xi_{i}\right) U\left[\sum_{r=1}^{\infty} a_{r}(t) \phi_{r}\left(\xi_{i}\right)+x_{s}(t)\right]=f_{k}(t),
$$




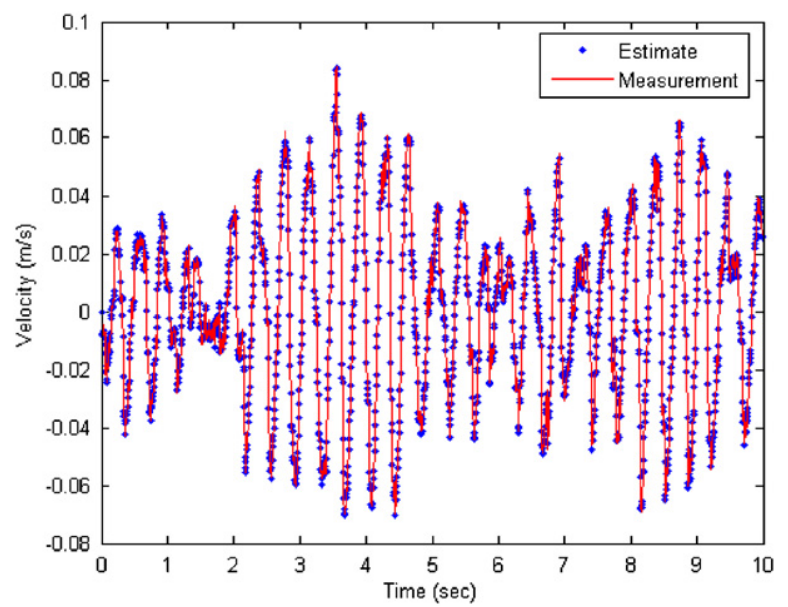

Fig. 16. Example 3: Estimation of the velocity state of the nonlinear sdof system with bilinear stiffness characteristics using bank of SIS filters.

$$
\begin{aligned}
f_{k}(t)= & -\ddot{x}_{s} \int_{0}^{L} m_{b} \phi_{k}(x) \mathrm{d} x-\dot{x}_{s} \int_{0}^{L} c \phi_{k}(x) \mathrm{d} x-\sum_{i=1}^{3} m_{i} \delta\left(x-\xi_{i}\right) \ddot{x}_{s}(t) \\
& -\sum_{i=1}^{3} k_{s i} x_{s}(t) U\left[\sum_{n=1}^{\infty} a_{n}(t) \phi_{n}\left(\xi_{i}\right)+x_{s}(t)\right] \phi_{k}\left(\xi_{i}\right), \quad k=1,2, \ldots \infty .
\end{aligned}
$$

For the purpose of illustration we truncate the infinite summation on the right-hand side of the above equation at the third term. By using the notation

$$
\left\{\begin{array}{llllll}
a_{1} & a_{2} & a_{3} & \dot{a}_{1} & \dot{a}_{2} & \dot{a}_{3}
\end{array}\right\}^{t}=\tilde{x}=\left\{\begin{array}{llllll}
x_{1} & x_{2} & x_{3} & x_{4} & x_{5} & x_{6}
\end{array}\right\}^{t}
$$

the governing equations for the generalized coordinates can be shown to be given by

$$
\begin{aligned}
\left\{\begin{array}{c}
\dot{x}_{k} \\
\dot{x}_{k+N}
\end{array}\right\} & =\left[\begin{array}{cc}
0_{3 \times 3} & I_{3 \times 3} \\
-\tilde{K}(\tilde{x}) & -\tilde{C}
\end{array}\right]\left\{\begin{array}{c}
x_{k} \\
x_{k+N}
\end{array}\right\} \mathrm{d} t+\left[\begin{array}{c}
0_{3 \times 1} \\
I_{3 \times 1}
\end{array}\right]\left\{f_{k}(\tilde{x}, t)\right\} \mathrm{d} t+\left[\begin{array}{c}
0_{3 \times 3} \\
\sigma_{3 \times 3}
\end{array}\right]\left\{\mathrm{d} B_{k}(t)\right\}, \quad k=1,2,3 \\
\tilde{K}_{l k} & =\omega_{k}^{2} \delta_{l k}+\sum_{i=1}^{3} k_{s i} U\left[\sum_{n=1}^{N} a_{n}(t) \phi_{n}\left(\xi_{i}\right)+x_{s}(t)\right]\left[\phi_{k}\left(\xi_{i}\right) \phi_{l}\left(\xi_{i}\right)\right], \quad l, k=1,2,3, \\
\tilde{C}_{n k} & =2 \eta_{n} \omega_{n} \delta_{n k}, \\
\sigma & =\left[\begin{array}{ccc}
\sigma_{1} & 0 & 0 \\
0 & \sigma_{2} & 0 \\
0 & 0 & \sigma_{3}
\end{array}\right] .
\end{aligned}
$$

Here we have now added, to the right-hand side, the random noise terms $\left\{\mathrm{d} B_{k}(t)\right\}$ which denote the process noise. It is assumed that the components of this vector random process are independent. The above equation can be discretized using the Ito-Taylor expansion as has been done in the previous example. For the purpose of illustration, we provide below the set of equations for the case of beam with only one cable support $\left(k_{s 1}=k_{s 3}=0, k_{s 2} \neq 0\right)$ :

$$
\begin{aligned}
x_{1(k+1)}= & x_{1 k}+x_{4 k} h+\left\{\left(-\omega_{1}^{2}-k_{s} U\left[Y_{k}\right] \chi_{11}\right) x_{1 k}-k_{s} U\left[Y_{k}\right] \chi_{12} x_{2 k}-k_{s} U\left[Y_{k}\right] \chi_{13} x_{3 k}-2 \eta \omega_{1} x_{4 k}\right. \\
& \left.+\ddot{x}_{s k} \beta_{1}-k_{s} U\left[Y_{k}\right] x_{s k} \phi_{1}\left(\xi_{2}\right)\right\}\left(h^{2} / 2\right)+\sigma_{1} I_{10},
\end{aligned}
$$


(a)

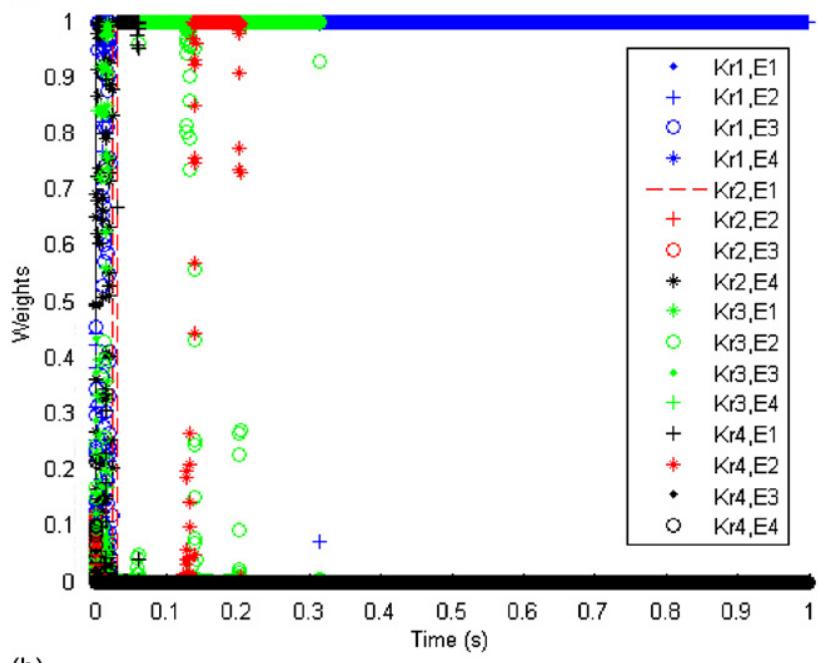

(b)

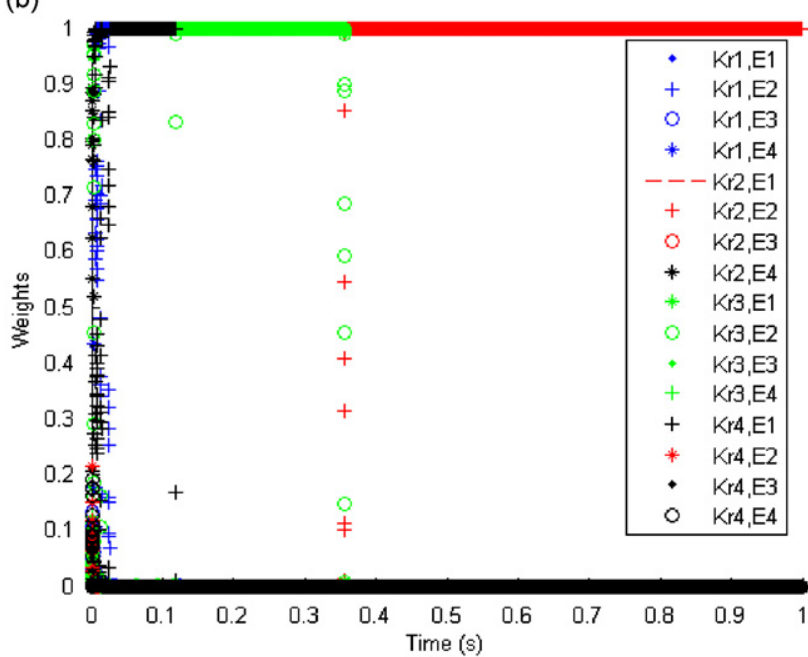

(c)

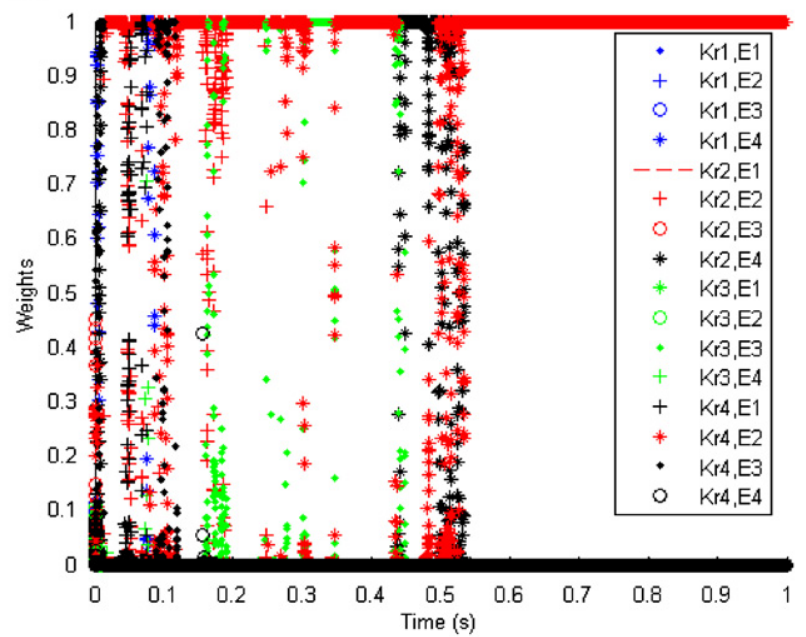

(d)

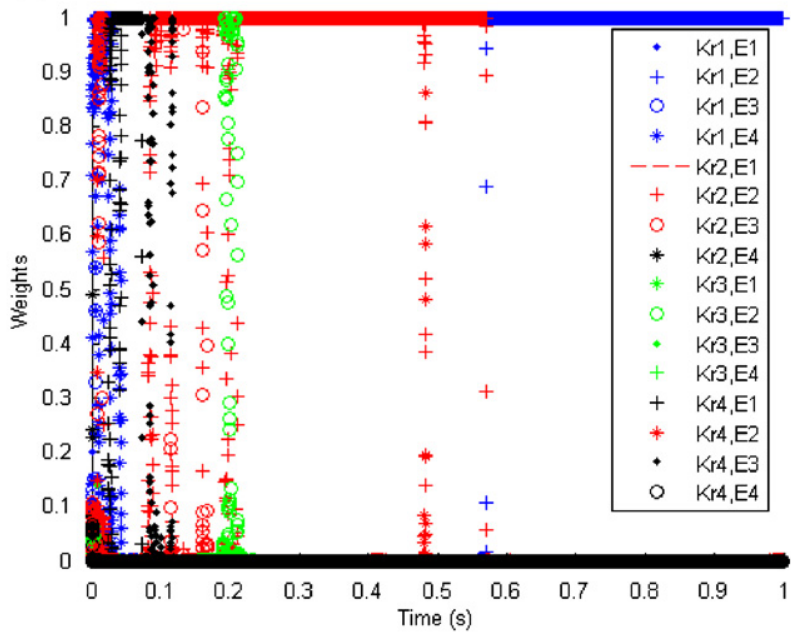

Fig. 17. Example 4.1: Estimation of rotary stiffness $K_{r}$ and elastic modulus $E$ of the beam using bank of SIS filters: (a)-(d) evolution of weights on $\left(K_{r}, E\right)$ for the first four cycles. The legends show the states of $K_{r}$ in Nm/rad and $E$ in N/m². The reference values of $K_{r}$ is $74.0025 \mathrm{Nm} / \mathrm{rad}$ and $E$ is $6.90 \times 10^{10} \mathrm{~N} / \mathrm{m}^{2}$. (a) Cycle $1-E_{1}=4.0 \times 10^{10} \mathrm{~N} / \mathrm{m}^{2} ; E_{2}=8.0 \times 10^{10} \mathrm{~N} / \mathrm{m}^{2} ; E_{3}=12.0 \times 10^{10} \mathrm{~N} / \mathrm{m}^{2}$; $E_{4}=16.0 \times 10^{10} \mathrm{~N} / \mathrm{m}^{2} ; K_{r 1}=50 \mathrm{Nm} / \mathrm{rad} ; K_{r 2}=100 \mathrm{Nm} / \mathrm{rad} ; K_{r 3}=150 \mathrm{Nm} / \mathrm{rad} ; K_{r 4}=200 \mathrm{Nm} / \mathrm{rad}$. The weight for $\left(K_{r 1}, E_{2}\right)$ is the highest. Number of samples used $=300$. (b) Cycle $2-E_{1}=5.5 \times 10^{10} \mathrm{~N} / \mathrm{m}^{2} ; \quad E_{2}=7 \times 10^{10} \mathrm{~N} / \mathrm{m}^{2} ; \quad E_{3}=8.5 \times 10^{10} \mathrm{~N} / \mathrm{m}^{2}$; $E_{4}=10.0 \times 10^{10} \mathrm{~N} / \mathrm{m}^{2} ; K_{r 1}=40 \mathrm{Nm} / \mathrm{rad} ; K_{r 2}=60 \mathrm{Nm} / \mathrm{rad} ; K_{r 3}=80 \mathrm{Nm} / \mathrm{rad} ; K_{r 4}=100 \mathrm{Nm} / \mathrm{rad}$. The weight for $\left(K_{r 2}, E_{2}\right)$ is the highest. Number of samples used 500 . (c) Cycle $3-E_{1}=6.0 \times 10^{10} \mathrm{~N} / \mathrm{m}^{2} ; E_{2}=7.0 \times 10^{10} \mathrm{~N} / \mathrm{m}^{2} ; E_{3}=8.0 \times 10^{10} \mathrm{~N} / \mathrm{m}^{2} ; E_{4}=9.0 \times 10^{10} \mathrm{~N} / \mathrm{m}^{2}$; $K_{r 1}=50 \mathrm{Nm} / \mathrm{rad} ; K_{r 2}=60 \mathrm{Nm} / \mathrm{rad} ; K_{r 3}=70 \mathrm{Nm} / \mathrm{rad} ; K_{r 4}=80 \mathrm{Nm} / \mathrm{rad}$. The weight for $\left(K_{r 4}, E_{2}\right)$ is the highest. Number of samples used $=600$. (d) Cycle $4-E_{1}=6.5 \times 10^{10} \mathrm{~N} / \mathrm{m}^{2} ; E_{2}=7.0 \times 10^{10} \mathrm{~N} / \mathrm{m}^{2} ; E_{3}=7.5 \times 10^{10} \mathrm{~N} / \mathrm{m}^{2} ; E_{4}=8.0 \times 10^{10} \mathrm{~N} / \mathrm{m}^{2} ; K_{r 1}=70 \mathrm{Nm} / \mathrm{rad}$; $K_{r 2}=75 \mathrm{Nm} / \mathrm{rad} ; K_{r 3}=80 \mathrm{Nm} / \mathrm{rad} ; K_{r 4}=85 \mathrm{Nm} / \mathrm{rad}$. The weight $\left(K_{r 1}, E_{2}\right)$ is the highest. The number of samples used $=700$.

$$
\begin{aligned}
x_{2(k+1)}= & x_{2 k}+x_{5 k} h+\left\{-k_{s} U\left[Y_{k}\right] \chi_{21} x_{1 k}+\left(-\omega_{2}^{2}-k_{s} U\left[Y_{k}\right] \chi_{22}\right) x_{2 k}-k_{s} U\left[Y_{j}\right] \chi_{23} x_{3 k}-2 \eta \omega_{2} x_{5 k}\right. \\
& \left.+\ddot{x}_{s k} \beta_{2}-k_{s} U\left[Y_{k}\right] x_{s k} \phi_{2}\left(\xi_{2}\right)\right\}\left(h^{2} / 2\right)+\sigma_{2} I_{20},
\end{aligned}
$$

$$
\begin{aligned}
x_{3(k+1)}= & x_{3 k}+x_{6 k} h+\left\{-k_{s} U\left[Y_{k}\right] \chi_{31} x_{1 k}-k_{s} U\left[Y_{k}\right] \chi_{32} x_{2 k}+\left(-\omega_{3}^{2}-k_{s} U\left[Y_{k}\right] \chi_{33}\right) x_{3 k}\right. \\
& \left.-2 \eta \omega_{3} x_{6 k}+\ddot{x}_{s k} \beta_{3}-k_{s} U\left[Y_{k}\right] x_{s k} \phi_{3}\left(\xi_{2}\right)\right\}\left(h^{2} / 2\right)+\sigma_{3} I_{30},
\end{aligned}
$$




$$
\begin{aligned}
x_{4(k+1)}= & x_{4 k}+\left\{-x_{1 k} \omega_{1}^{2} h-x_{1 k} k_{s} U\left[Y_{k}\right] \chi_{11} h-k_{s} U\left[Y_{k}\right] \chi_{12} x_{2 k} h-k_{s} U\left[Y_{k}\right] \chi_{13} x_{3 k} h-2 \eta \omega_{1} x_{4 k} h\right. \\
& \left.+\beta_{1} v_{s k}-k_{s} U\left[Y_{k}\right] \phi_{1}\left(\xi_{2}\right) d_{s k}\right\}+\left\{x _ { 4 k } \left(-k_{s} \delta\left[Y_{k}\right] \phi_{1}\left(\xi_{2}\right) \chi_{11} x_{1 k}-\omega_{1}^{2}-k_{s} U\left[Y_{k}\right] \xi_{11}\right.\right. \\
& \left.-k_{s} \delta\left[Y_{k}\right] \phi_{1}\left(\xi_{2}\right) \xi_{12} x_{2 k}-k_{s} \delta\left[Y_{k}\right] \phi_{1}\left(\xi_{2}\right) \xi_{13} x_{3 k}-k_{s} \delta\left[Y_{k}\right] \phi_{1}\left(\xi_{2}\right)^{2} x_{s k}\right) \\
& +x_{5 k}\left(-k_{s} \delta\left[Y_{k}\right] \phi_{2}\left(\xi_{2}\right) \chi_{11} x_{1 k}-k_{s} \delta\left[Y_{k}\right] \phi_{2}\left(\xi_{2}\right) \chi_{12} x_{2 k}-k_{s} U\left[Y_{k}\right] \chi_{12}-k_{s} \delta\left[Y_{k}\right] \phi_{2}\left(\xi_{2}\right) \chi_{13} x_{3 k}\right. \\
& \left.-k_{s} \delta\left[Y_{k}\right] \phi_{2}\left(\xi_{2}\right) \phi_{1}\left(\xi_{2}\right) x_{s k}\right)+x_{6 k}\left(-k_{s} \delta\left[Y_{k}\right] \phi_{3}\left(\xi_{2}\right) \chi_{11} x_{1 k}-k_{s} \delta\left[Y_{k}\right] \phi_{3}\left(\xi_{2}\right) \chi_{12} x_{2 k}\right. \\
& \left.-k_{s} \delta\left[Y_{k}\right] \phi_{3}\left(\xi_{2}\right) \chi_{13} x_{3 k}-k_{s} U\left[Y_{k}\right] \chi_{13}-k_{s} \delta\left[Y_{k}\right] \phi_{3}\left(\xi_{2}\right) \phi_{1}\left(\xi_{2}\right) x_{s k}\right)-2\left(\left(-\omega_{1}^{2}-k_{s} U\left[Y_{k}\right] \chi_{11}\right) x_{1 k}\right. \\
& \left.\left.-k_{s} U\left[Y_{k}\right] \chi_{12} x_{2 k}-k_{s} U\left[Y_{k}\right] \chi_{13} x_{3 k}-2 \eta \omega_{1} x_{4 k}+\ddot{x}_{s k} \beta_{1}-k_{s} U\left[Y_{k}\right] x_{s k} \phi_{1}\left(\xi_{2}\right)\right) \eta \omega_{1}\right\}\left(h^{2} / 2\right) \\
& -2 \eta \omega_{1} \sigma_{1} I_{10}+I_{1},
\end{aligned}
$$

$$
\begin{aligned}
x_{5(k+1)}= & x_{5 k}-\left\{k_{s} U\left[Y_{k}\right] \chi_{21} x_{1 k} h+x_{2 k} \omega_{2}^{2} h+x_{2 k} k_{s} U\left[Y_{k}\right] \chi_{22} h+k_{s} U\left[Y_{k}\right] \chi_{23} x_{3 k} h+2 \eta \omega_{2} x_{5 k} h-\beta_{2} v_{s k}\right. \\
& \left.+k_{s} U\left[Y_{k}\right] \phi_{2}\left(\xi_{2}\right) d_{s k}\right\}+\left\{x _ { 4 k } \left(-k_{s} \delta\left[Y_{k}\right] \phi_{1}\left(\xi_{2}\right) \chi_{21} x_{1 k}-k_{s} U\left[Y_{k}\right] \chi_{21}-k_{s} \delta\left[Y_{k}\right] \phi_{1}\left(\xi_{2}\right) \chi_{22} x_{2 k}\right.\right. \\
& \left.-k_{s} \delta\left[Y_{k}\right] \phi_{1}\left(\xi_{2}\right) \chi_{23} x_{3 k}-k_{s} \delta\left[Y_{k}\right] \phi_{1}\left(\xi_{2}\right) x_{s k} \phi_{2}\left(\xi_{2}\right)\right)+x_{5 k}\left(-k_{s} \delta\left[Y_{k}\right] \phi_{2}\left(\xi_{2}\right) \chi_{21} x_{1 k}\right. \\
& \left.-k_{s} \delta\left[Y_{k}\right] \phi_{2}\left(\xi_{2}\right) \chi_{22} x_{2 k}-\omega_{2}^{2}-k_{s} U\left[Y_{k}\right] \chi_{22}-k_{s} \delta\left[Y_{k}\right] \phi_{2}\left(\xi_{2}\right) \chi_{23} x_{3 k}-k_{s} \delta\left[Y_{k}\right] \phi_{2}\left(\xi_{2}\right)^{2} x_{s k}\right) \\
& +x_{6 k}\left(-k_{s} \delta\left[Y_{k}\right] \phi_{3}\left(\xi_{2}\right) \chi_{21} x_{1 k}-k_{s} \delta\left[Y_{k}\right] \phi_{3}\left(\xi_{2}\right) \chi_{22} x_{2 k}-k_{s} \delta\left[Y_{k}\right] \phi_{3}\left(\xi_{2}\right) \chi_{23} x_{3 k}-k_{s} U\left[Y_{k}\right] \chi_{23}\right. \\
& \left.-k_{s} \delta\left[Y_{k}\right] x_{s k} \phi_{2}\left(\xi_{2}\right) \phi_{3}\left(\xi_{2}\right)\right)-2\left(-k_{s} U\left[Y_{k}\right] \chi_{21} x_{1 k}+\left(-\omega_{2}^{2}-k_{s} U\left[Y_{k}\right] \chi_{22}\right) x_{2 k}-k_{s} U\left[Y_{k}\right] \chi_{23} x_{3 k}\right. \\
& \left.\left.-2 \eta \omega_{2} x_{5 k}+\ddot{x}_{s k} \omega_{2}-k_{s} U\left[Y_{k}\right] x_{s k} \phi_{2}\left(\xi_{2}\right)\right) \eta \omega_{2}\right\}\left(h^{2} / 2\right)-2 \eta \omega_{2} \sigma_{2} I_{20}+\sigma_{2} I_{2},
\end{aligned}
$$

$$
\begin{aligned}
x_{6(k+1)}= & x_{6 k}-\left\{k_{s} U\left[Y_{k}\right] \chi_{31} x_{1 k} h+k_{s} U\left[Y_{k}\right] \chi_{32} x_{2 k} h+x_{3 k} \omega_{3}^{2} h+x_{3 k} k_{s} U\left[Y_{k}\right] \chi_{33} h+2 \eta \omega_{3} x_{6 k} h\right. \\
& \left.-\beta_{3} v_{s k}+k_{s} U\left[Y_{k}\right] \phi_{3}\left(\xi_{2}\right) d_{s k}\right\}+\left\{x _ { 4 k } \left(-k_{s} \delta\left[Y_{k}\right] \phi_{1}\left(\xi_{2}\right) \chi_{31} x_{1 k}-k_{s} U\left[Y_{k}\right] \chi_{31}\right.\right. \\
& \left.-k_{s} \delta\left[Y_{k}\right] \phi_{1}\left(\xi_{2}\right) \chi_{32} x_{2 k}-k_{s} \delta\left[Y_{k}\right] \phi_{1}\left(\xi_{2}\right) \chi_{33} x_{3 k}-k_{s} \delta\left[Y_{k}\right] \phi_{1}\left(\xi_{2}\right) x_{s k} \phi_{3}\left(\xi_{2}\right)\right) \\
& +x_{5 k}\left(-k_{s} \delta\left[Y_{k}\right] \phi_{2}\left(\xi_{2}\right) \chi_{31} x_{1 k}-k_{s} \delta\left[Y_{k}\right] \phi_{2}\left(\xi_{2}\right) \chi_{32} x_{2 k}-k_{s} U\left[Y_{k}\right] \chi_{32}-k_{s} \delta\left[Y_{k}\right] \phi_{2}\left(\xi_{2}\right) \chi_{33} x_{3 k}\right. \\
& \left.-k_{s} \delta\left[Y_{k}\right] \phi_{2}\left(\xi_{2}\right) x_{s k} \phi_{3}\left(\xi_{2}\right)\right) \\
& +x_{6 k}\left(-k_{s} \delta\left[Y_{k}\right] \phi_{3}\left(\xi_{2}\right) \chi_{31} x_{1 k}-k_{s} \delta\left[Y_{k}\right] \phi_{3}\left(\xi_{2}\right) \chi_{32} x_{2 k}-k_{s} \delta\left[Y_{k}\right] \phi_{3}\left(\xi_{2}\right) \chi_{33} x_{3 k}-\omega_{3}^{2}-k_{s} U\left[Y_{k}\right] \chi_{33}\right. \\
& \left.-k_{s} \delta\left[Y_{k}\right] x_{s k} \phi_{3}\left(\xi_{2}\right)^{2}\right)-2\left(-k_{s} U\left[Y_{k}\right] \chi_{31} x_{1 k}-k_{s} U\left[Y_{k}\right] \chi_{32} x_{2 k}+\left(-\omega_{3}^{2}-k_{s} U\left[Y_{k}\right] \chi_{33}\right) x_{3 k}\right. \\
& \left.-2 \eta \omega_{3} x_{6 k}+\ddot{x}_{s k} \beta_{3}-k_{s} U\left[Y_{k}\right] x_{s k} \phi_{3}\left(\xi_{2}\right) \eta \omega_{3}\right\}\left(h^{2} / 2\right)-2 \eta \omega_{3} \sigma_{3} I_{30}+\sigma_{3} I_{3},
\end{aligned}
$$

with

$$
\begin{aligned}
& m(x)=m_{b}+\sum_{i=1}^{3} m_{i} \delta\left(x-\xi_{i}\right), \\
& \beta_{1}=\int_{0}^{L} m(x) \phi_{1}(x) \mathrm{d} x ; \quad \beta_{2}=\int_{0}^{L} m(x) \phi_{2}(x) \mathrm{d} x, \quad \beta_{3}=\int_{0}^{L} m(x) \phi_{3}(x) \mathrm{d} x, \\
& \chi=\left[\begin{array}{ccc}
\phi_{1}^{2}\left(x_{2}\right) & \phi_{2}\left(x_{2}\right) \phi_{1}\left(x_{2}\right) & \phi_{3}\left(x_{2}\right) \phi_{1}\left(x_{2}\right) \\
\phi_{1}\left(x_{2}\right) \phi_{2}\left(x_{2}\right) & \phi_{2}^{2}\left(x_{2}\right) & \phi_{3}\left(x_{2}\right) \phi_{2}\left(x_{2}\right) \\
\phi_{1}\left(x_{2}\right) \phi_{3}\left(x_{2}\right) & \phi_{3}\left(x_{2}\right) \phi_{2}\left(x_{2}\right) & \phi_{3}^{2}\left(x_{2}\right)
\end{array}\right], \\
& \chi_{11}=\chi(1,1), \quad \chi_{12}=\chi(1,2), \quad \chi_{13}=\chi(1,3), \quad \chi_{21}=\chi(2,1), \quad \chi_{22}=\chi(2,2) \text {, } \\
& \chi_{23}=\chi(2,3), \quad \chi_{31}=\chi(3,1), \quad \chi_{32}=\chi(3,2), \quad \chi_{33}=\chi(3,3), \\
& Y_{j}=\left(x_{1 j} \phi_{1}\left(\xi_{2}\right)+x_{2 j} \phi_{2}\left(\xi_{2}\right)+x_{3 j} \phi_{3}\left(\xi_{2}\right)+x_{3 j}\right) \text {. }
\end{aligned}
$$


In this example also the drift terms contains Heaveside's step function which is not differentiable when the argument of this function goes to zero. Consequently, in discretized equations (49) we have formally introduced the Dirac delta functions to indicate this fact. In the treatment of these terms in the numerical work, we follow the procedure that has already been described while discussing the previous example. The data in the numerical work are: $E=6.90 \times 10^{10} \mathrm{~N} / \mathrm{m}^{2}, I=5.759 \times 10^{-11} \mathrm{~m}^{4}, m_{b}=0.16246 \mathrm{~kg} / \mathrm{m}, L=0.537 \mathrm{~m}$, $m_{1}=19.835 \times 10^{-3} \mathrm{~kg}, \quad m_{2}=19.825 \times 10^{-3} \mathrm{~kg}, \quad m_{3}=15.32 \times 10^{-3} \mathrm{~kg}, \quad \xi_{1}=0.175 \mathrm{~m}, \quad \xi_{2}=0.352 \mathrm{~m}$, $\xi_{3}=0.527 \mathrm{~m}, \eta_{i}=0.02$ for $i=1,2,3, K_{r}=74.0025 \mathrm{Nm} / \mathrm{rad}$, and $k_{s}=3.50 \times 10^{4} \mathrm{~N} / \mathrm{m}$. We consider the support displacement as given by Eq. (29) and for numerical work we take $\omega_{0}=32 \mathrm{rad} / \mathrm{s}, n=10$, and amplitude of support displacement is taken as $0.001 \mathrm{~m}$. Within the framework of this problem we configure different examples. In all the examples, the measurements are synthetically generated.

\subsection{Example 4.1: Beam without any cables}

Here we take $k_{s i}=0$ for $i=1,2,3$, i.e., the beam now behaves as a linear system. The process equation (49) gets accordingly simplified considerably. The parameters to be identified are taken to be the beam elastic modulus $E$ and the rotary stiffness $K_{r}$. The measurements are taken to be made on acceleration at two points on the beam and, accordingly, the measurement equation is obtained as

$$
\left\{\begin{array}{l}
y_{1 k} \\
y_{2 k}
\end{array}\right\}=\left[\begin{array}{lll}
\phi_{1}\left(\xi_{2}\right) & \phi_{2}\left(\xi_{2}\right) & \phi_{3}\left(\xi_{2}\right) \\
\phi_{1}\left(\xi_{3}\right) & \phi_{2}\left(\xi_{3}\right) & \phi_{3}\left(\xi_{3}\right)
\end{array}\right]\left[\begin{array}{ll}
-\tilde{K} & -\tilde{C}
\end{array}\right]\left\{\begin{array}{c}
x_{1 k} \\
x_{2 k} \\
x_{3 k} \\
x_{4 k} \\
x_{5 k} \\
x_{6 k}
\end{array}\right\}+\left\{\begin{array}{l}
\ddot{x}_{s k} \\
\ddot{x}_{s k}
\end{array}\right\}+\left\{\begin{array}{l}
\sigma_{m 1} v_{1 k} \\
\sigma_{m 2} v_{2 k}
\end{array}\right\} .
$$

It is to be noted that, in this example, we are illustrating how the SIS bank of filters approach could be used to identify more than one system parameters. In the numerical work, the measurements are generated with reference values of $E=6.90 \times 10^{10} \mathrm{~N} / \mathrm{m}^{2}$ and $K_{r}=74.0025 \mathrm{Nm} / \mathrm{rad}$. This data is contaminated with measurement noise having zero mean and standard deviations $\sigma_{m 1}=0.7508 \mathrm{~m} / \mathrm{s}^{2}$ and $\sigma_{m 2}=0.7386 \mathrm{~m} / \mathrm{s}^{2}$. The process noises considered here are additive in nature with standard deviations $\sigma_{1}=\sigma_{2}=\sigma_{3}=0.0404 \mathrm{~N}$. The results on system parameters identified and the estimated states are shown in Figs. 17-19. The parameters $\left(K_{r}, E\right)$ are initially taken to lie, respectively, in the ranges $50-200 \mathrm{Nm} / \mathrm{rad}$ and $4 \times 10^{10}-16 \times 10^{10} \mathrm{~N} / \mathrm{m}^{2}$. This pair of random variables are taken to have sixteen distinct states and initially these states is taken to have same weights. At the end of fourth cycle of filtering with 700 particles, the range of parameters narrows down to $70-85 \mathrm{Nm} / \mathrm{rad}$ and $6.5 \times 10^{10}-8 \times 10^{10} \mathrm{~N} / \mathrm{m}^{2}$. The associated plots of expected values and variances of $K_{r}$ and $E$ are shown in Fig. 18(a)-(d). It is observed that the filter estimates the expected value of $70.0 \mathrm{Nm} / \mathrm{rad}$ for $K_{r}$ and $7.0 \times 10^{10} \mathrm{~N} / \mathrm{m}^{2}$ for $E$ which compare favorably with respective reference values of these parameters. The plots of estimated acceleration response at points of measurement are observed to compare well with the corresponding measurements as shown in Figs. 19(a) and (b).

\subsection{Example 4.2: Beam suspended on one cable}

Here we assume that the beam is suspended on cable at $x=\xi_{2}$. The measurements are taken to be made on displacement and velocity near the tip of the beam and accordingly the measurement equations are obtained as

$$
\left\{\begin{array}{l}
y_{1 k} \\
y_{2 k}
\end{array}\right\}=\left[\begin{array}{cccccc}
\phi_{1}\left(\xi_{3}\right) & \phi_{2}\left(\xi_{3}\right) & \phi_{3}\left(\xi_{3}\right) & 0 & 0 & 0 \\
0 & 0 & 0 & \phi_{1}\left(\xi_{3}\right) & \phi_{2}\left(\xi_{3}\right) & \phi_{3}\left(\xi_{3}\right)
\end{array}\right]\left\{\begin{array}{c}
x_{1 k} \\
x_{2 k} \\
x_{3 k} \\
x_{4 k} \\
x_{5 k} \\
x_{6 k}
\end{array}\right\}+\left\{\begin{array}{l}
x_{s k} \\
\dot{x}_{s k}
\end{array}\right\}+\left\{\begin{array}{l}
\sigma_{m 1} v_{1 k} \\
\sigma_{m 2} v_{2 k}
\end{array}\right\} .
$$


(a)

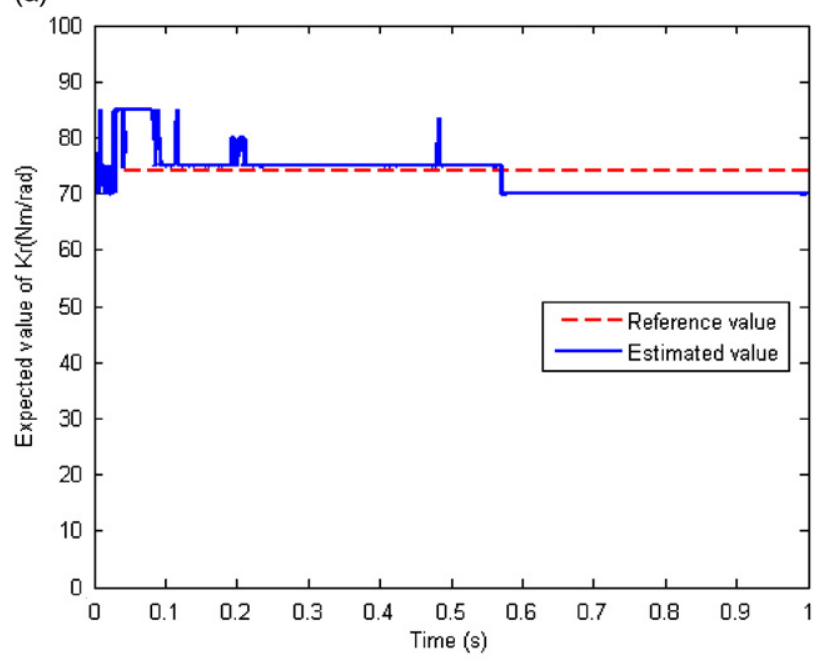

(b)

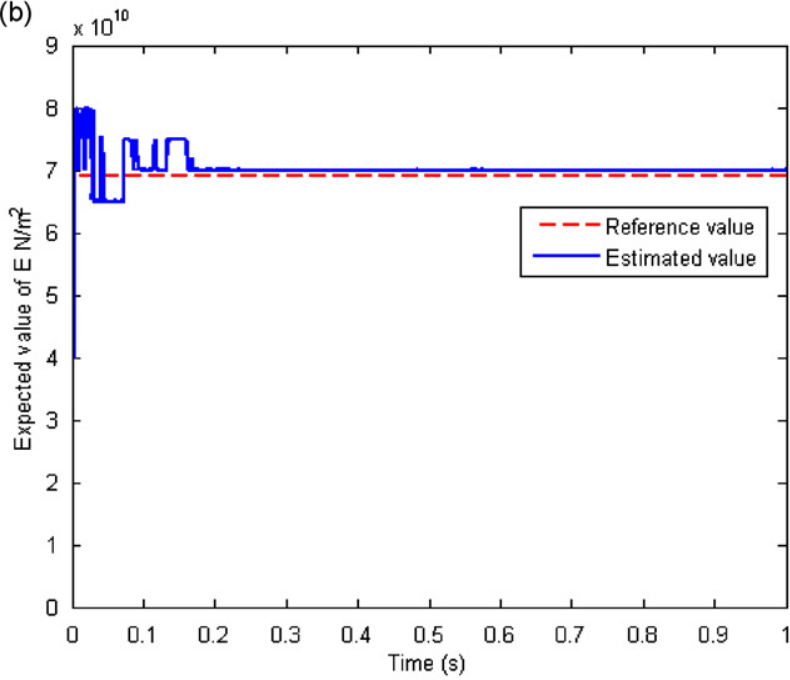

(c)

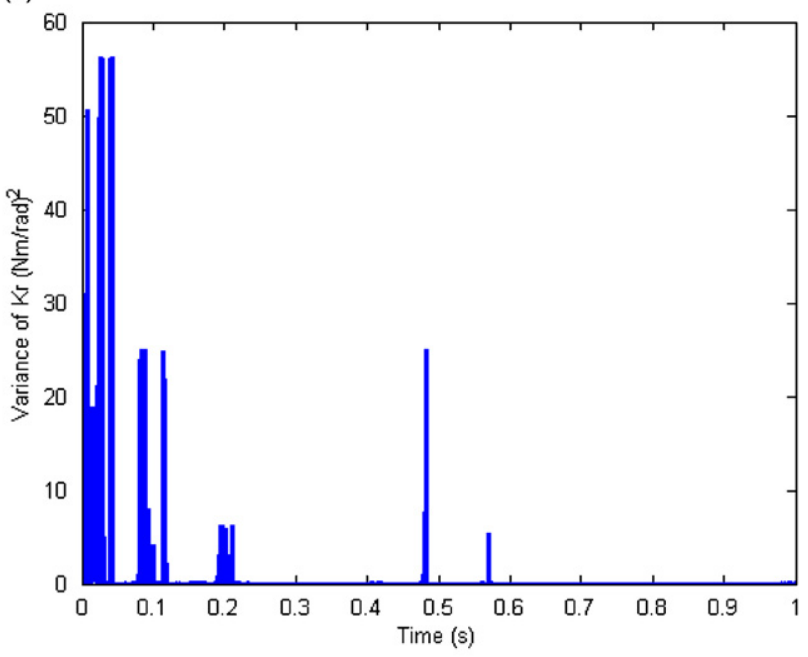

(d)

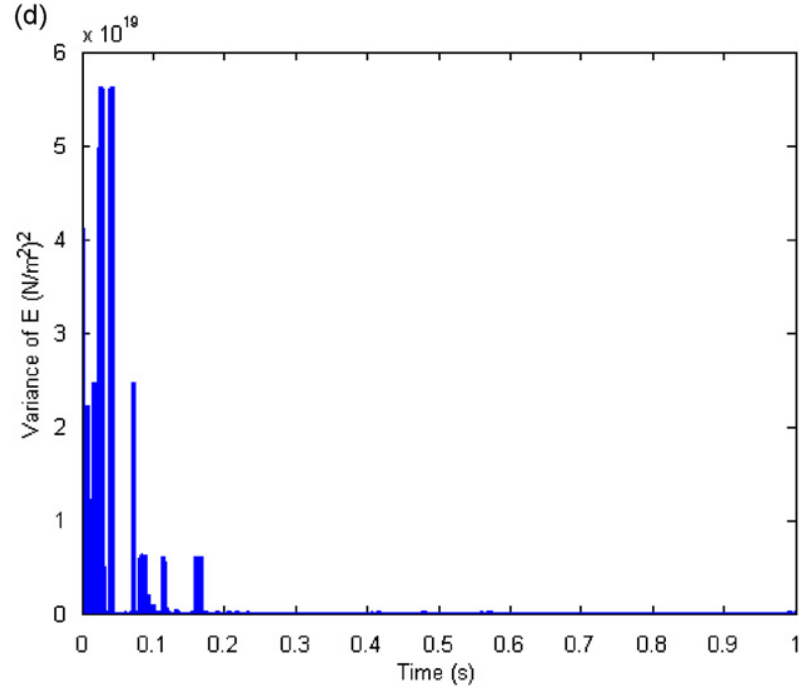

Fig. 18. Example 4.1: Estimation of rotary stiffness $K_{r}$ and elastic modulus $E$ of the beam using bank of SIS filters: (a) expected value of $K_{r}$, (b) expected value of $E$, (c) variance of $K_{r}$ and (d) variance of $E$. The reference values of $K_{r}$ is $74.0025 \mathrm{Nm} / \mathrm{rad}$ and $E$ is $6.90 \times 10^{10} \mathrm{~N} / \mathrm{m}^{2}$.

The parameter to be identified is taken to be the cable stiffness $k_{s}$. In the numerical work, the measurements are generated with a reference value of $k_{s}=3.5 \times 10^{4} \mathrm{~N} / \mathrm{m}$. These data are seeded with measurement noise having zero means and standard deviations $\sigma_{m 1}=3.909 \times 10^{-5} \mathrm{~m}$ and $\sigma_{m 2}=0.0015 \mathrm{~m} / \mathrm{s}$. The process noises considered here are additive in nature with standard deviations $\sigma_{1}=\sigma_{2}=\sigma_{3}=0.01 \mathrm{~N}$. The results on estimation on cable stiffness and system states are shown in Figs. 20-22. From Figs. 20 and 21, it is evident that the algorithm is successfully predicting the cable stiffness in five cycles of global iterations. The stiffness parameter $k_{s}$ is initially taken to lie in the range $2 \times 10^{4}-5 \times 10^{4} \mathrm{~N} / \mathrm{m}$, which, at the end of fifth cycle of filtering with 1000 particles, narrows down to the range $3.30 \times 10^{4}-3.60 \times 10^{4} \mathrm{~N} / \mathrm{m}$. Thus, we get the estimate for the expected value of cable stiffness to be about $3.40 \times 10^{4} \mathrm{~N} / \mathrm{m}$ which compares very well with the reference value of $k_{s}=3.5 \times 10^{4} \mathrm{~N} / \mathrm{m}$. The plots of estimated displacement and velocity response at point of measurements are again observed to compare well with the corresponding measurements in Figs. 22(a) and (b). 
(a)

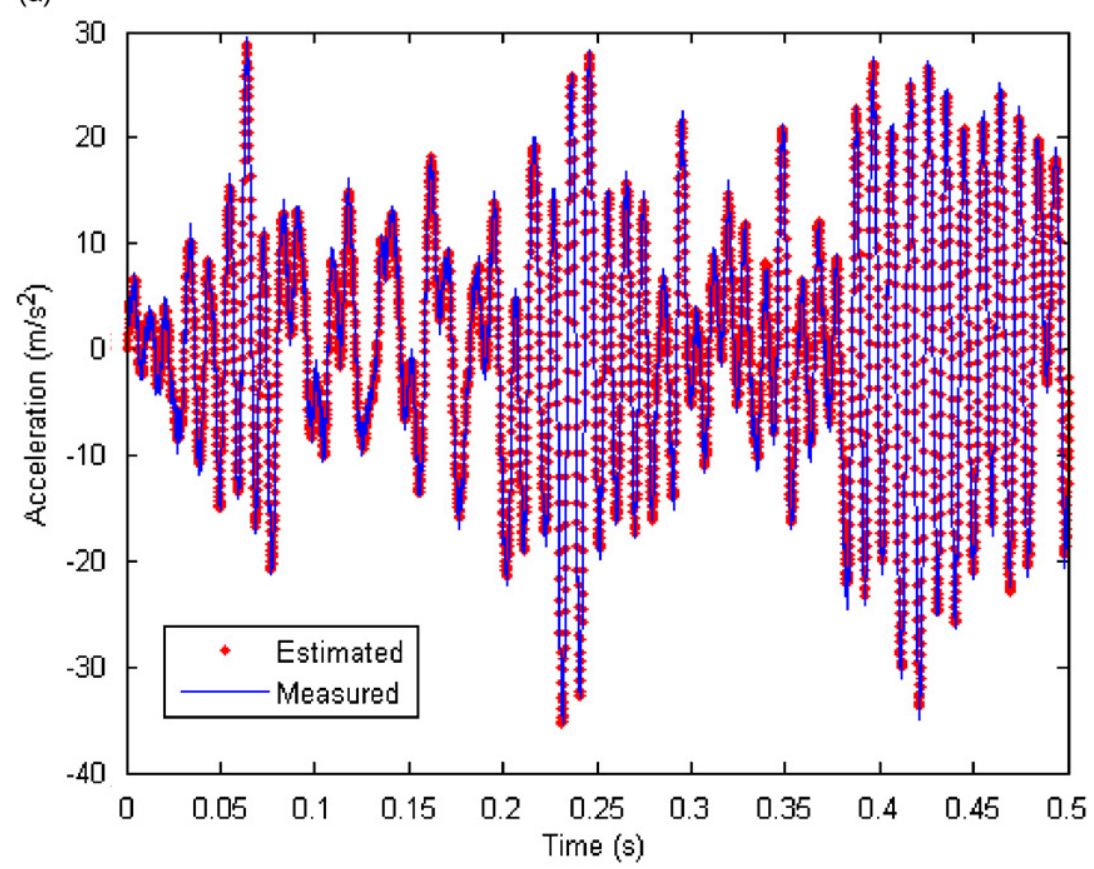

(b)

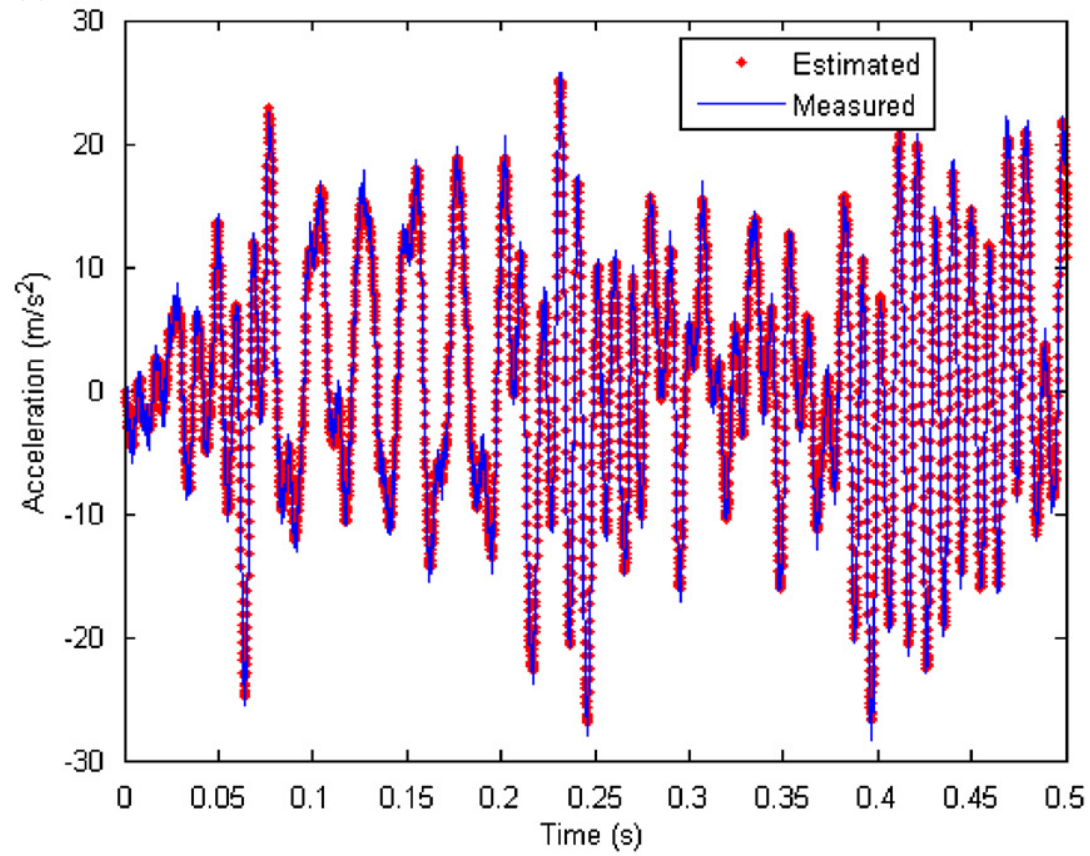

Fig. 19. Example 4.1: Estimation of the acceleration response of the beam at (a) $x=\xi_{2}$ and (b) $x=\xi_{3}$ using bank of SIS filters.

\section{Discussion and conclusions}

The present study proposes a bank of particle filters approach to address problems of nonlinear structural system identification. The basic methods of particle filtering for problems of dynamic 
(a)

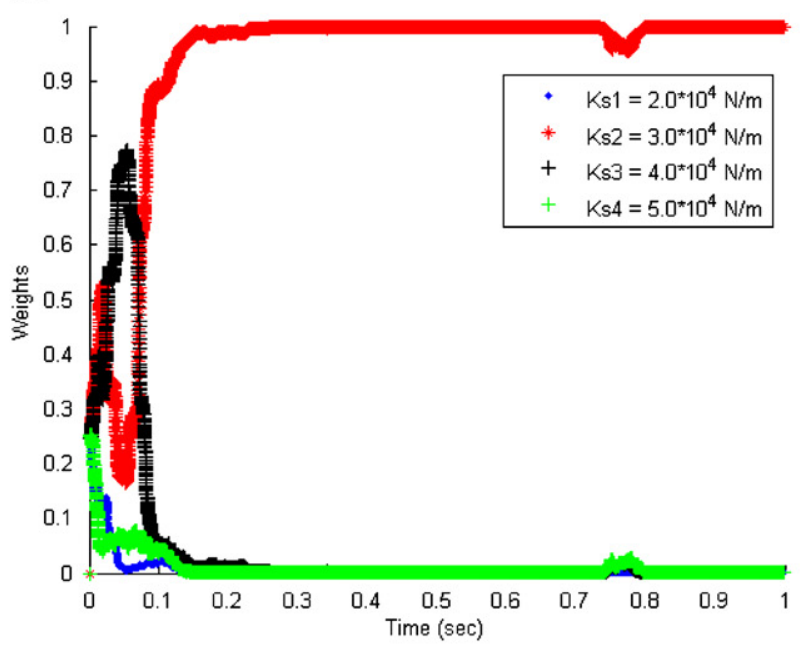

(b)

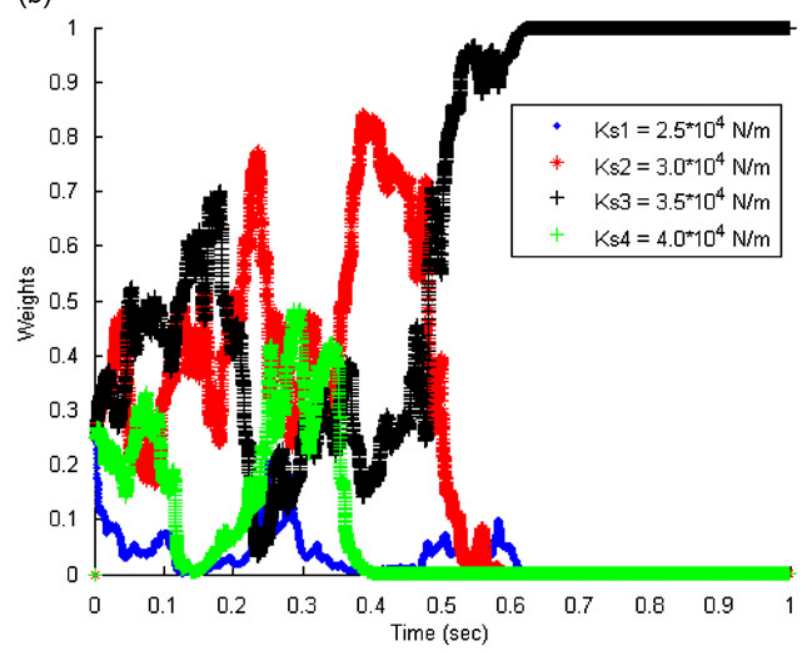

(c)

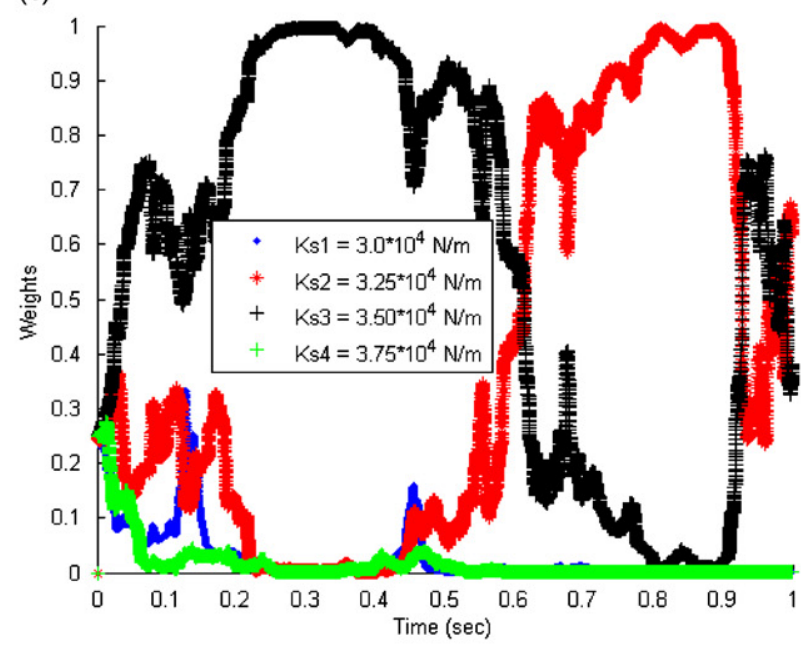

(d)

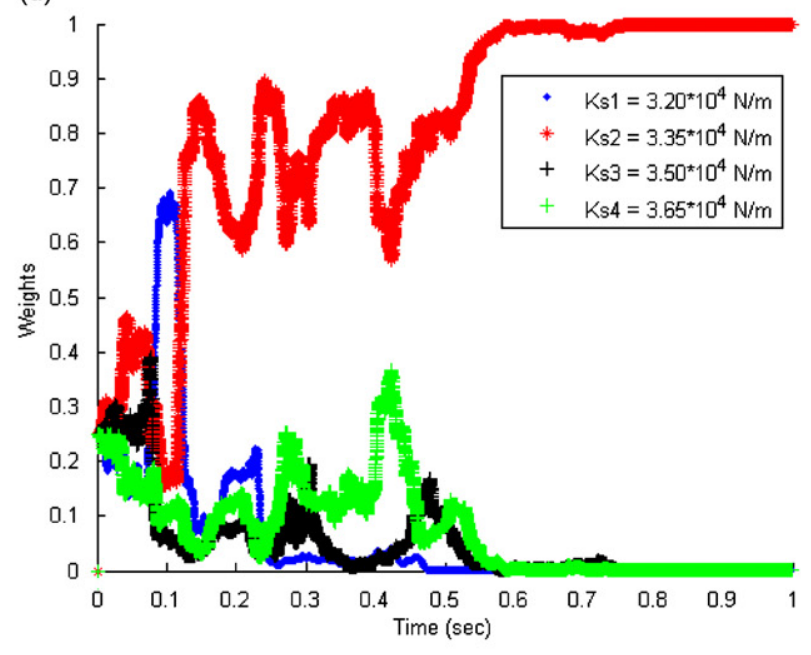

(e)

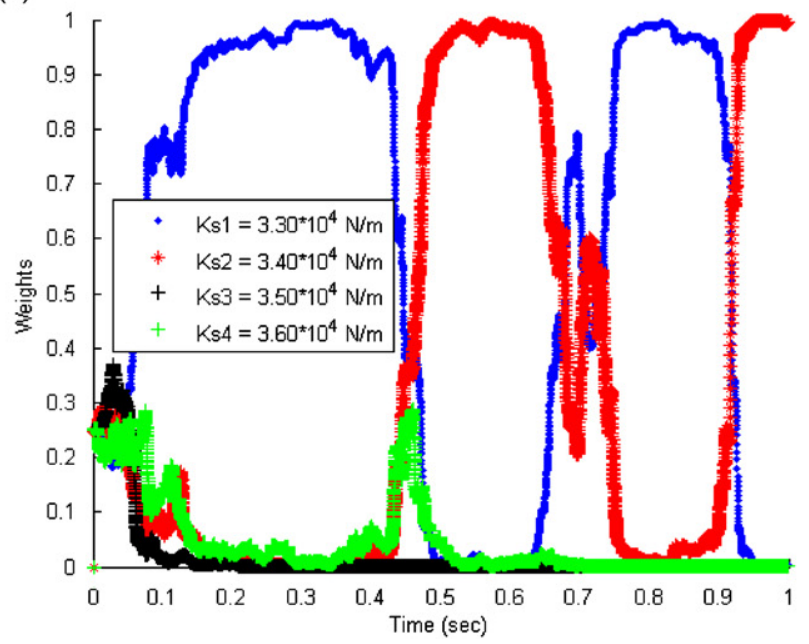

Fig. 20. Example 4.2: Estimation of cable stiffness parameter $k_{s}$ of the beam suspended on cable at $x=\xi_{2}$ using bank of SIS filters: (a)-(e) evolution of weights on $k_{s}$ for the first five cycles. The legends show the states of $k_{s}$ in $\mathrm{N} / \mathrm{m}$. Reference value of $k_{s}$ is $3.50 \times 10^{4} \mathrm{~N} / \mathrm{m}$. (a) cycle 1 , (b) cycle 2 , (c) cycle 3 , (d) cycle 4 , (e) cycle 5 . 

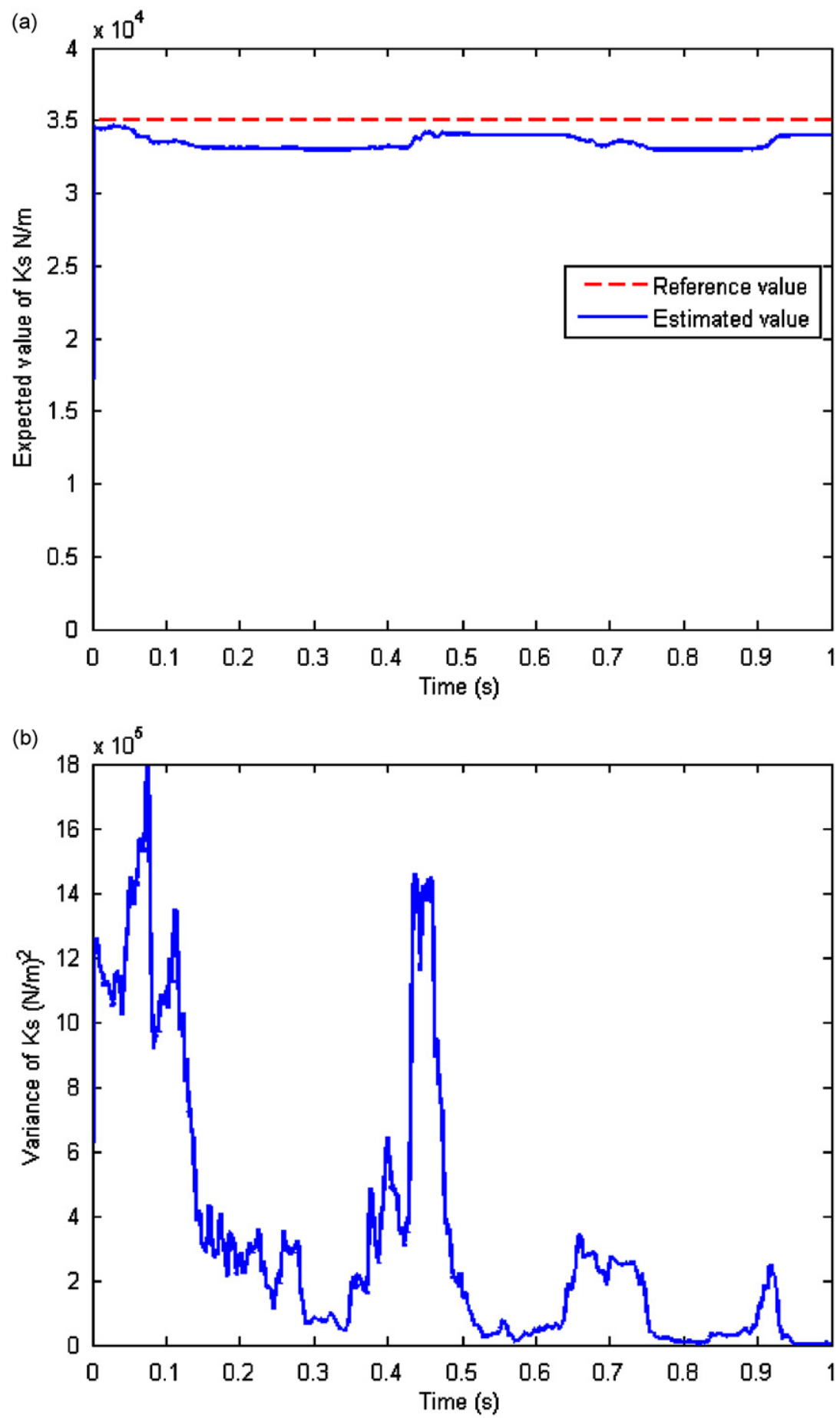

Fig. 21. Example 4.2: Estimation of cable stiffness parameter $k_{s}$ of the beam suspended on cable at $x=\xi_{2}$ using bank of SIS filters: (a) expected value of $k_{s}$, (b) variance of $k_{s}$. Reference value of $k_{s}$ is $3.50 \times 10^{4} \mathrm{~N} / \mathrm{m}$.

state estimation are readily available in the existing literature related to the areas of economics, object tracking, digital communication channels and signal processing. Their application to problems of structural engineering is presently an area that is being researched upon in the structural engineering 

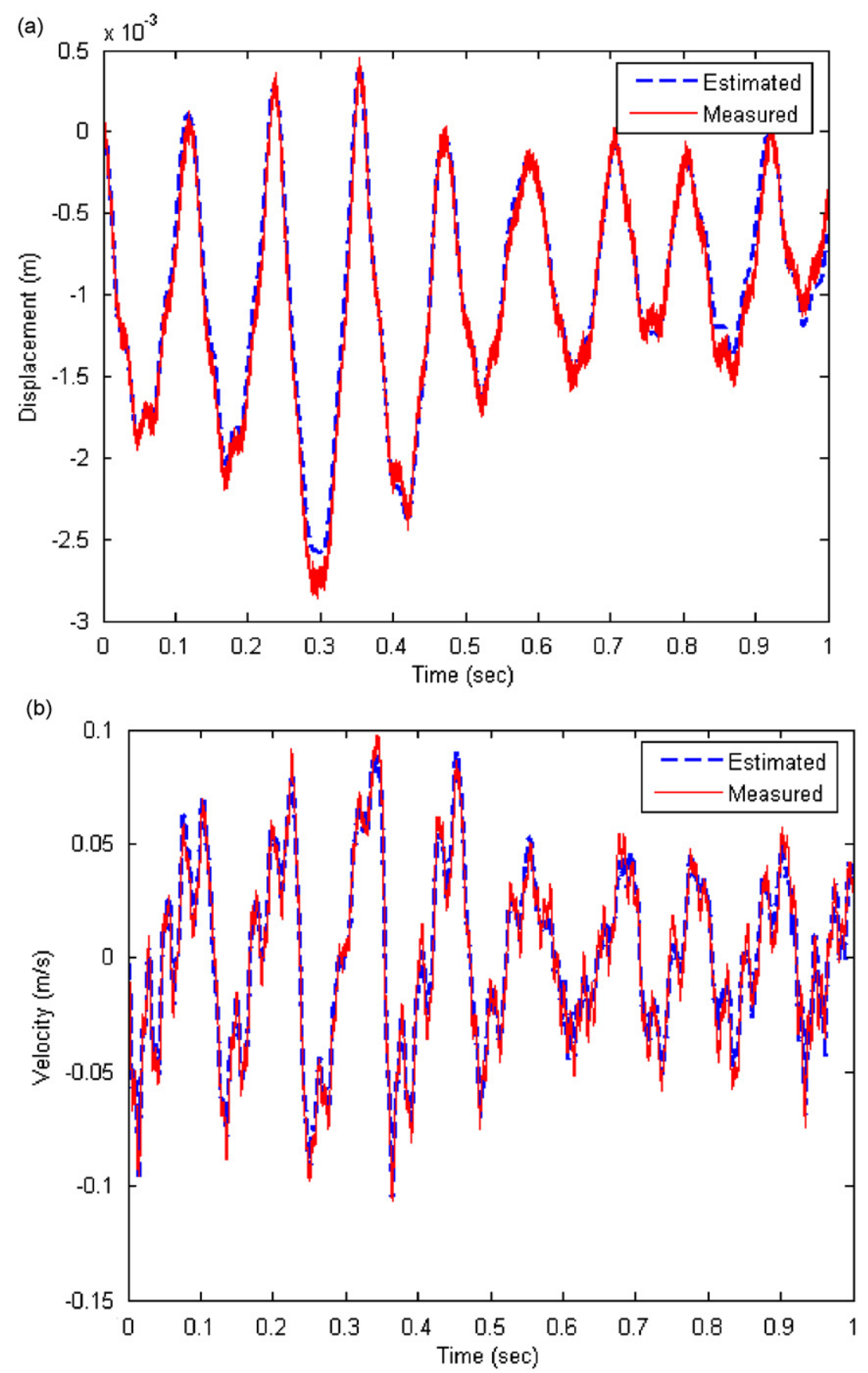

Fig. 22. Example 4.2: Estimation of the displacement and velocity response of the beam suspended on cable at $x=\xi_{2}$ using bank of SIS filters: (a) displacement response at $x=\xi_{3}$, (b) velocity response at $x=\xi_{3}$.

community. The present work contributes to this effort. Some of the characteristic features of the method are as follows:

- The study is formulated in the time-domain which is the most natural domain for treatment of nonlinear dynamical systems; i.e., the procedures developed for solving the governing process equations retain the pathwise fidelity of the response trajectories with respect to the system initial conditions. 
- The approach is formulated within the framework of probabilistic modeling, and, consequently, the presence of uncertainties due to measurement noise and the effects of unmodeled dynamics are taken into account in a rational manner.

- The framework provides a systematic means to deal with the incompatibility in the sizes of analytical and measurements models. Thus, no approximate model reduction/expansion schemes, such as those based on condensation techniques, are employed here.

- An a priori model for the system states is formulated using the laws of mechanics of the system. The information contained in the measurements is assimilated into the evolution of states within a Bayesian framework. The present study is based on the assumption that the process noise and measurement noise are additive Gaussian white noise sequences. Since the structural systems under study are taken to be nonlinear, the process equation is nonlinear in the state variables. The present study is based on the assumption that the measurement process is linear in the system states. The system parameters to be identified are declared as a state of discrete random variables with finite number of states. For each possible realization of these random variables, one particle filter is employed to estimate the states. Thus, we obtain a bank of particle filters which, upon assimilating the information contained in the measurements, also leads to weights associated with realization of system parameters to be identified. The present paper makes contribution to the development of the algorithm to evaluate the evolution of these weights in a recursive manner within the framework of Monte Carlo simulation-based filtering.

The method of adaptive filtering, in the present study, has been applied to situations in which system parameters are identified in an offline manner. The proposed approach, which involves global iterations, essentially helps in narrowing down the hypothesized range of states of the system parameters in successive iterations. This also helps us to quickly check if the initial hypothesized range of the system parameters to be identified indeed encloses the value of system parameters from which the measurement emanates. Thus, an inappropriate choice for the range would be detected at the end of first cycle of iteration itself, thereby, enabling the corrective step to be taken without expending any further computational effort. To illustrate this point, we consider Example 2. Here measurement is obtained with $\alpha=3.45 \times 10^{5} \mathrm{~N} / \mathrm{m}^{3}$. If initial discretization of $\alpha$ into four states $\alpha=1.25 \times 10^{5}, 1.75 \times 10^{5}, 2.25 \times 10^{5}$ and $2.75 \times 10^{5} \mathrm{~N} / \mathrm{m}^{3}$ is made, the resulting evolution of weights is as in Fig. 23. Since the initially assumed range of $\alpha$ does not include the reference value of $\alpha$, we observed that the weights associated with $\alpha=2.75 \times 10^{5} \mathrm{~N} / \mathrm{m}^{3}$ (which lies closest to the reference value of $\alpha$ ) shows the highest weight. This provides the prompt that hypothesized range of $\alpha$ is inappropriate.

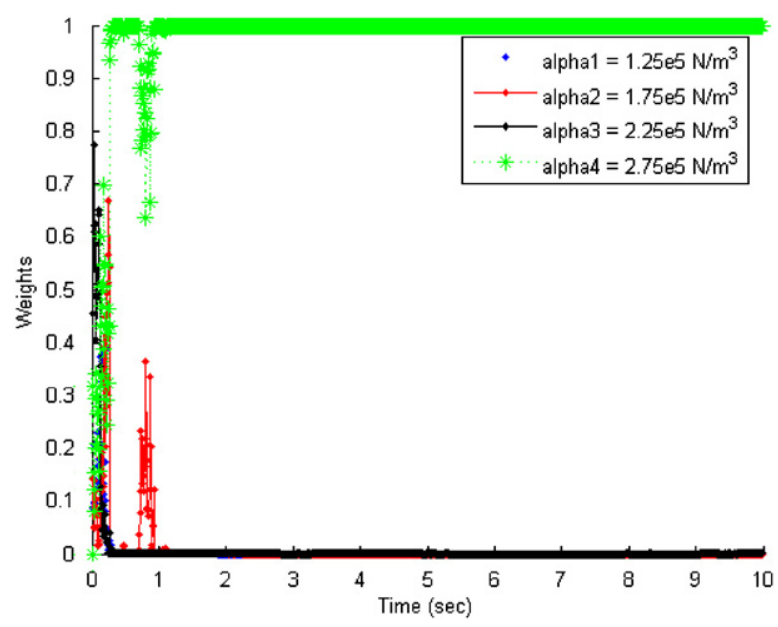

Fig. 23. Example 2: Estimation of nonlinear stiffness parameter $\alpha$ using bank of SIS filters: evolution of weights on $\alpha$. The legends show the states of $\alpha$ in N/m $3 \mathrm{~m}^{3}$. Reference value of $\alpha$ is $3.45 \times 10^{5} \mathrm{~N} / \mathrm{m}^{3}$. 


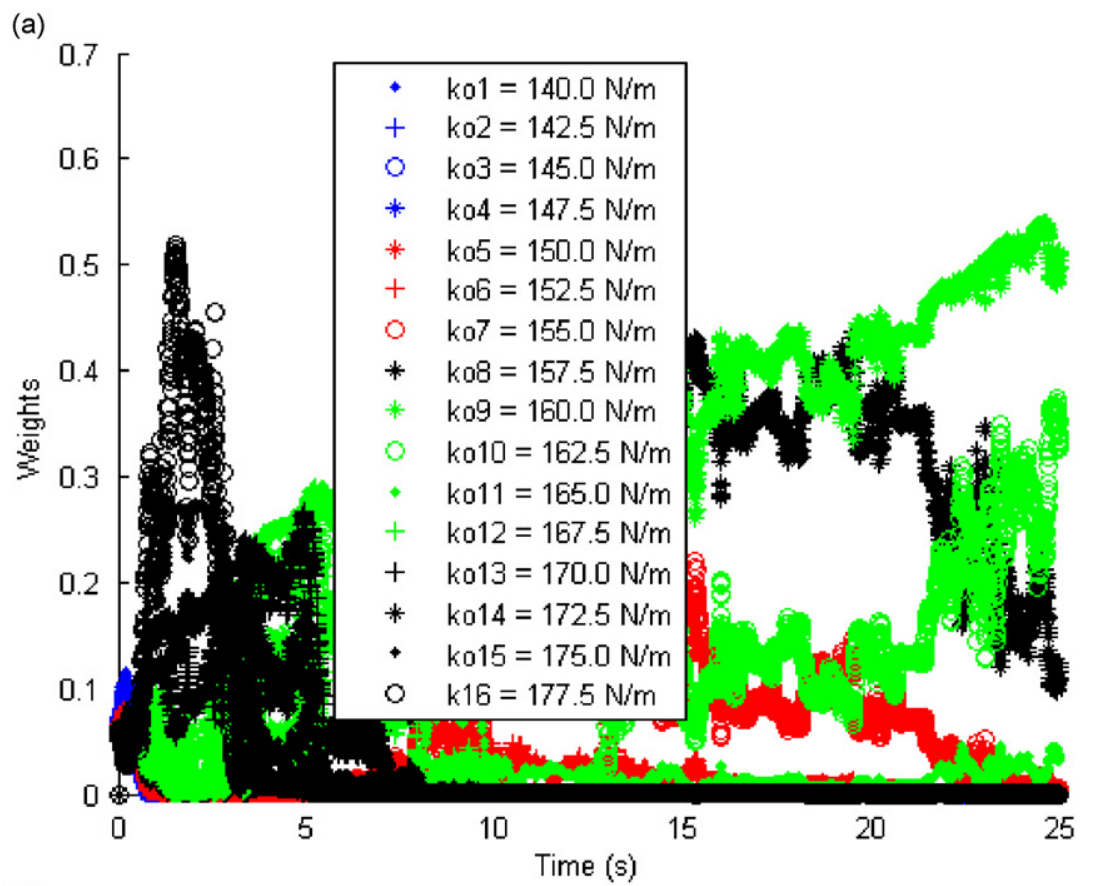

(b)

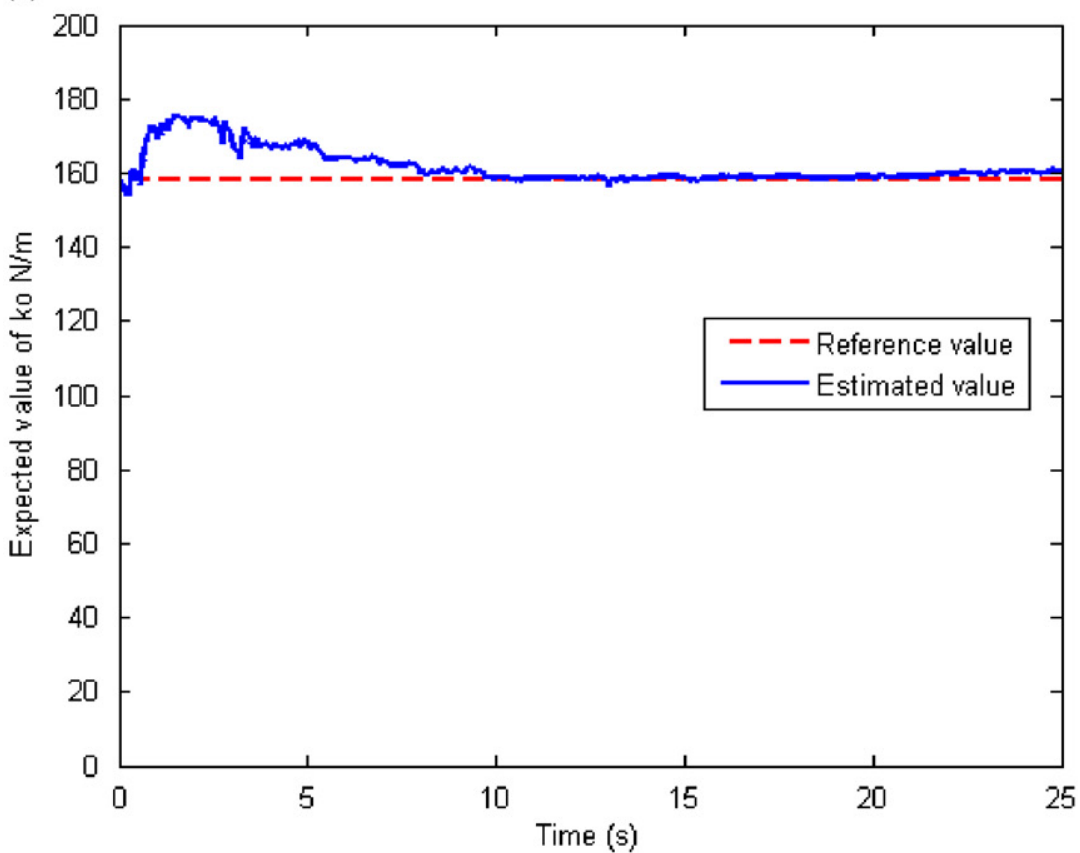

Fig. 24. Example 1: Estimation of system stiffness parameter $k_{o}$ using bank of filters approach (with sixteen filters running in parallel): (a) evolution of weights on the stiffness parameter $k_{o}$, the legends show the states of $k_{o}$ in $\mathrm{N} / \mathrm{m}$, (b) expected value of $k_{o}$. Reference value of $k_{o}$ is $157.91 \mathrm{~N} / \mathrm{m}$.

The global iteration strategy used in the present study is clearly not applicable if the parameters are to be identified in an online manner. In such applications, it could be necessary to discretize the system parameter range into a large number of states, so that a desired level of resolution in the parameter estimation is possible. To illustrate this, we consider Example 1 with reference value of $k_{o}=157.91 \mathrm{~N} / \mathrm{m}$. A bank of filters with sixteen hypothesized states for $k_{o}$ given by $140,142.5,145,147.5,150,152.5,155,157.5,160,162.5,165,167.5$, 


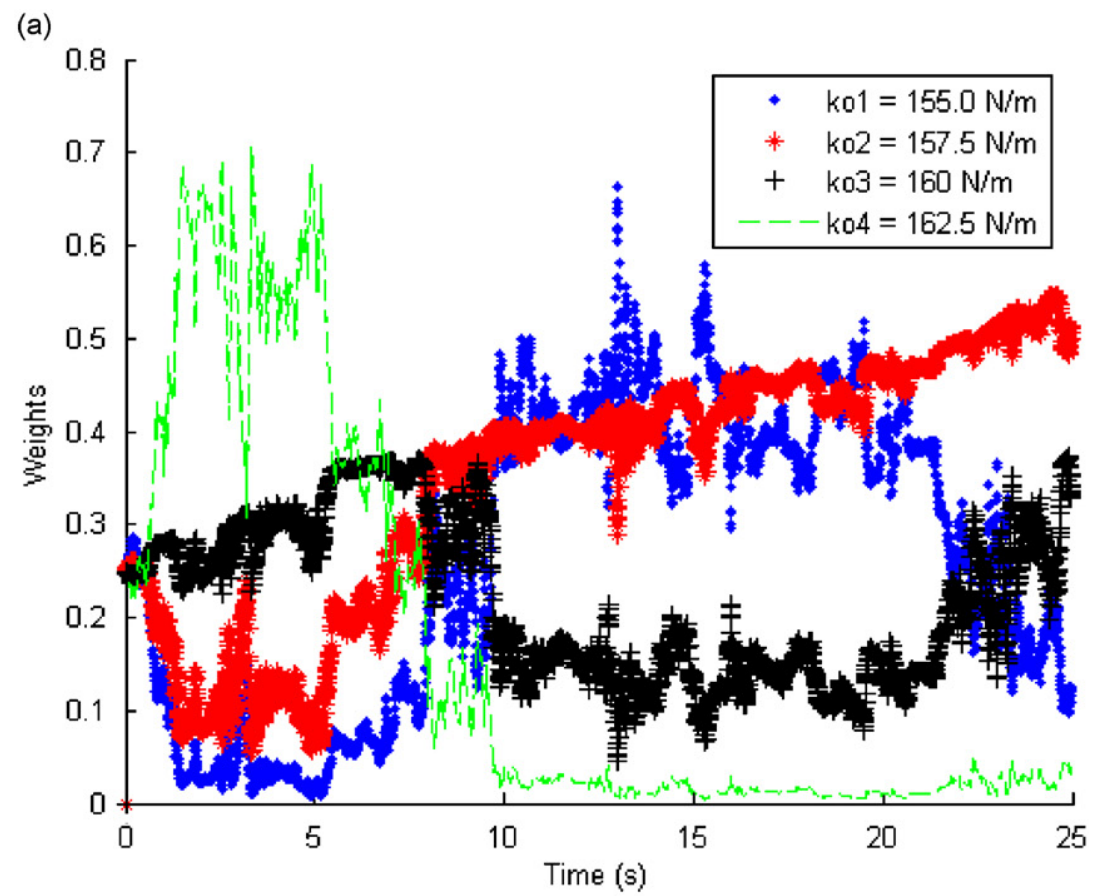

(b)

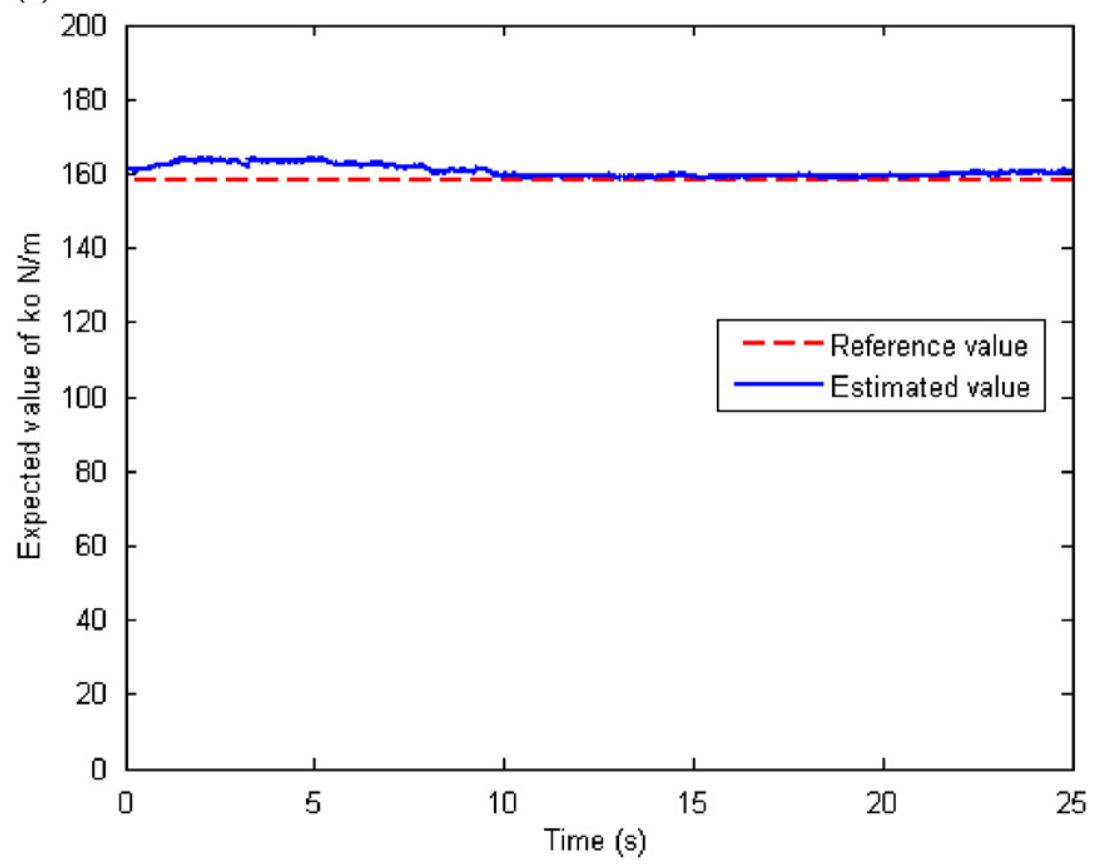

Fig. 25. Example 1: Estimation of system stiffness parameter $k_{o}$ using bank of filters approach: (a) evolution of weights on the stiffness parameter $k_{o}$. Legends show the states of $k_{o}$ in $\mathrm{N} / \mathrm{m}$. (b) Expected value of $k_{o}$. Reference value of $k_{o}$ is $157.91 \mathrm{~N} / \mathrm{m}$.

170, 172.5, 175, and $177.5 \mathrm{~N} / \mathrm{m}$ leads to the evolution of associated weights as shown in Fig. 24(a). The corresponding plot of expected value of $k_{o}$ is shown in Fig. 24(b). An alternative strategy with four cycles of iteration starting with four number of states for $k_{o}=155,157.5,160$, and $162.5 \mathrm{~N} / \mathrm{m}$ was also implemented and these results are shown in Fig. 25(a) and (b). As may be observed that the results shown in Figs. 24(b) and 25(b) lead to final estimates of $k_{o}$, and then compare well. 


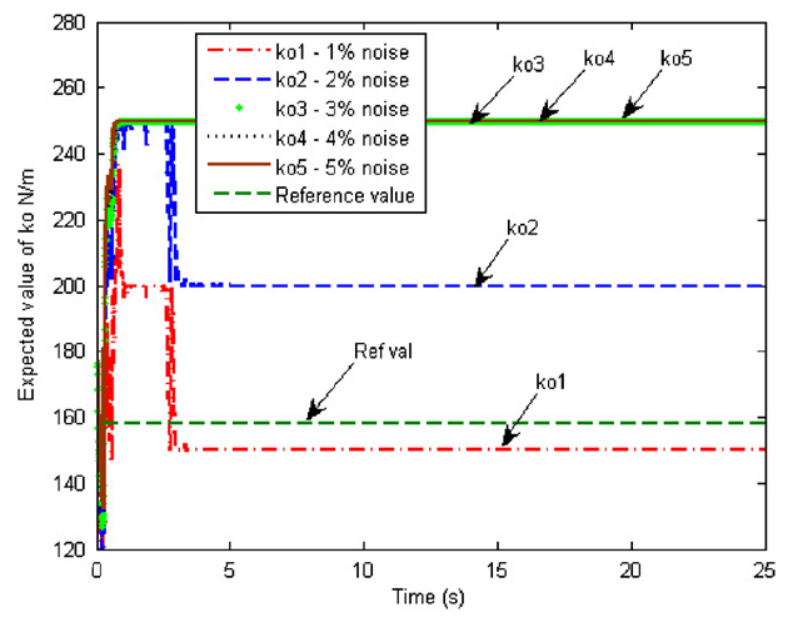

Fig. 26. Example 1: Estimation of expected value of the parameter $k_{o}$ using bank of Kalman filters. The legends show the measurement noise levels. Reference value of $k_{o}$ is $157.91 \mathrm{~N} / \mathrm{m}$.

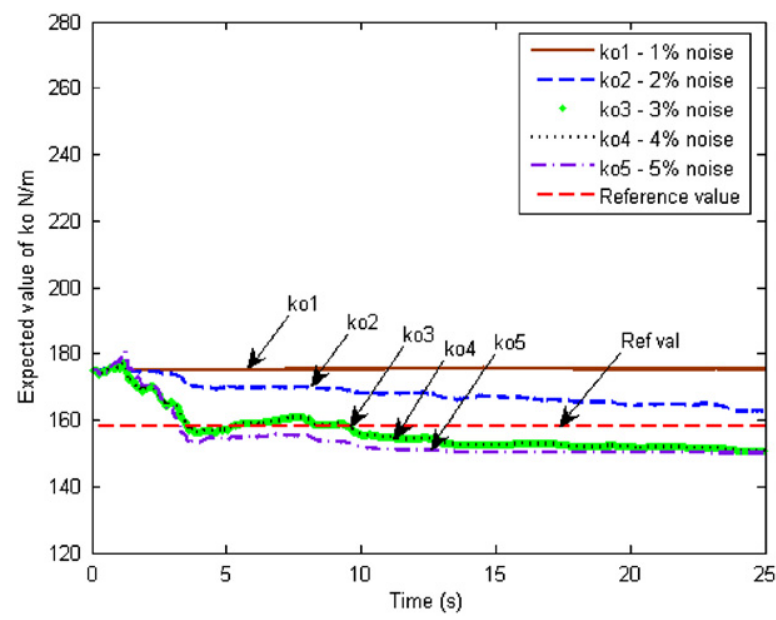

Fig. 27. Example 1: Estimation of expected value of the parameter $k_{o}$ using bank of SIS filters. The legends show the measurement noise levels. Reference value of $k_{o}$ is $157.91 \mathrm{~N} / \mathrm{m}$.

During the implementation of the bank of particle filters approach, it was observed that the filters performed relatively better for higher level of measurement noise. Conversely, the performance of bank of Kalman filters was found to deteriorate with an increase in variance of the measurement noise. To illustrate this, we applied these two approaches to the problem of estimation of stiffness of a linear single degree of freedom system (data as per Example 1), and Figs. 26 and 27 show the results obtained. It is observed that the results on the expected value of the system stiffness obtained using bank of Kalman filters (Fig. 26) compare well with the reference value of $k_{o}=157.9137 \mathrm{~N} / \mathrm{m}$ when the measurement noise is relatively small, while a converse situation is observed when the similar result is obtained using the bank of SIS filters.

As the numbers of system parameters to be identified becomes large, the bank of particle filters approach becomes computationally intensive. This difficulty possibly could be overcome if strategies based on space filling lattice hypercube sampling are combined with adaptive filtering strategies. In the present study, we have illustrated the identification procedure by using synthetically generated measurement data. The performance of proposed method clearly needs to be assessed when measurements are actually obtained from laboratory or field measurements. An immediate problem that would arise in such a situation is associated with the assessment of process noise that represents the effects of unmodeled dynamics. A way to overcome this 
difficulty would be to treat the process noise parameters also as parameters to be identified and carry out the system parameters and noise parameters identification problems in a unified manner. Studies aimed at addressing these issues are currently being conducted by the present authors.

\section{Acknowledgement}

The work reported in this paper has been supported by funding from the Aeronautical Research and Development Board, Government of India. The authors sincerely thank the funding agency for the support offered.

\section{Appendix A. Estimation of $\hat{I}\left[x_{0: k}\left(\theta_{i}\right)\right]$ using the sequential importance sampling particle filter}

The procedure we propose to use for estimating $\hat{I}\left[x_{0: k}\left(\theta_{i}\right)\right]$ is as outlined by Doucet [23]. For simplicity, we omit to explicitly state the dependence of the estimate on $\theta$ and write $\hat{I}\left[x_{0: k}\right]$ in place of $\hat{I}\left[x_{0: k}\left(\theta_{i}\right)\right]$. The basic idea consists here in choosing an importance sampling pdf, denoted by $\pi\left(x_{0: k} \mid y_{1: k}\right)$, such that, if $p\left(x_{0: k} \mid y_{1: k}\right)>0 \Rightarrow \pi\left(x_{0: k} \mid y_{1: k}\right)>0$. In this case, the integral in Eq. (19) can be written as

$$
\begin{aligned}
I\left[x_{0: k}\right] & =\int x_{0: k} \frac{p\left(x_{0: k} \mid y_{1: k}\right)}{\pi\left(x_{0: k} \mid y_{1: k}\right)} \pi\left(x_{0: k} \mid y_{1: k}\right) \mathrm{d} x_{0: k} \\
& =\int x_{0: k} w^{*}\left(x_{0: k}\right) \pi\left(x_{0: k} \mid y_{1: k}\right) \mathrm{d} x_{0: k}=E_{\pi}\left[x_{0: k} w^{*}\left(x_{0: k}\right) \mid y_{1: k}\right] .
\end{aligned}
$$

Here $E_{\pi}[\cdot]$ denotes expectation with respect to the pdf $\pi\left(x_{0: k} \mid y_{1: k}\right)$. If $\left\{x_{i, 0: n}\right\}_{i=1}^{N}$ are samples drawn from $\pi\left(x_{0: k} \mid y_{1: k}\right)$, and

$$
\begin{aligned}
\hat{I}_{N}\left[x_{0: k}\right] & =\frac{1}{N} \sum_{i=1}^{N} x_{i, 0: k} w^{*}\left(x_{i, 0: k}\right) \quad \text { with } \\
w^{*}\left(x_{i, 0: k}\right) & =\frac{p\left(x_{i, 0: k} \mid y_{1: k}\right)}{\pi\left(x_{i, 0: k} \mid y_{1: k}\right)}=\frac{p\left(y_{1: k} \mid x_{i, 0: k}\right) p\left(x_{i, 0: k}\right)}{\pi\left(x_{i, 0: k} \mid y_{1: k}\right) p\left(y_{1: k}\right)}=\frac{p\left(y_{1: k} \mid x_{i, 0: k}\right) p\left(x_{i, 0: k}\right)}{\pi\left(x_{i, 0: k} \mid y_{1: k}\right) \int p\left(y_{1: k} \mid x_{0: k}\right) p\left(x_{0: k}\right) \mathrm{d} x_{0: k}}
\end{aligned}
$$

it can be shown that $\lim _{N \rightarrow \infty} \hat{I}_{N}\left[x_{0: k}\right] \rightarrow I\left[x_{0: k}\right]$ almost surely [23]. It may be emphasized that the evaluation of the normalization constant $p\left(y_{1: k}\right)=\int p\left(y_{1: k} \mid x_{0: k}\right) p\left(x_{0: k}\right) \mathrm{d} x_{0: k}$ in Eq. (A.2) presents a major difficulty in implementing the above procedure since it is seldom possible to express this constant in a closed form. However, if one evaluates this integral too via importance sampling, one gets,

$$
\hat{I}_{N}\left[x_{0: k}\right]=\frac{(1 / N) \sum_{i=1}^{N} x_{i, 0: k}\left(\left(p\left(y_{1: k} \mid x_{i, 0: k}\right) p\left(x_{i, 0: k}\right)\right) /\left(\pi\left(x_{i, 0: k} \mid y_{1: k}\right)\right)\right)}{(1 / N) \sum_{j=1}^{N}\left(\left(p\left(y_{1: k} \mid x_{j, 0: k}\right) p\left(x_{j, 0: k}\right)\right) /\left(\pi\left(x_{j, 0: k} \mid y_{1: k}\right)\right)\right)}=\frac{\sum_{i=1}^{N} x_{i, 0: k} w\left(x_{i, 0: k}\right)}{\sum_{j=1}^{N} w\left(x_{j, 0: k}\right)}=\sum_{i=1}^{N} x_{i, 0: k} \tilde{w}\left(x_{i, 0: k}\right)
$$

with the new weights $\tilde{w}\left(x_{i, 0: k}\right)$ given by

$$
\tilde{w}\left(x_{i, 0: k}\right)=\frac{w\left(x_{i, 0: k}\right)}{\sum_{j=1}^{N} w\left(x_{j, 0: k}\right)} \quad \text { with } \quad w\left(x_{i, 0: k}\right)=\frac{p\left(y_{1: k} \mid x_{i, 0: k}\right) p\left(x_{i, 0: k}\right)}{\pi\left(x_{i, 0: k} \mid y_{1: k}\right)} .
$$

If one selects the importance sampling density function of the form

$$
\pi\left(x_{0: k} \mid y_{1: k}\right)=\pi\left(x_{0: k-1} \mid y_{1: k}\right) \pi\left(x_{k} \mid x_{0: k-1}, y_{1: k}\right)
$$

it would then be possible to compute the importance weights in a recursive manner. To see this, we note that $p\left(y_{1: k} \mid x_{0: k}\right)=\prod_{j=1}^{k} p\left(y_{j} \mid x_{j}\right)$ and $p\left(x_{0: k}\right)=p\left(x_{0}\right) \prod_{j=1}^{k} p\left(x_{j} \mid x_{j-1}\right)$. Using these facts and Eq. (A.5) in Eq. (A.4), 
we get

$$
\begin{aligned}
w\left(x_{0: k}\right)= & \frac{\prod_{j=1}^{k} p\left(y_{j} \mid x_{j}\right) p\left(x_{0}\right) \prod_{j=1}^{k} p\left(x_{j} \mid x_{j-1}\right)}{\pi\left(x_{0: k-1} \mid y_{1: k}\right) \pi\left(x_{k} \mid x_{0: k-1}, y_{1: k}\right)} \\
= & \frac{\prod_{j=1}^{k-1} p\left(y_{j} \mid x_{j}\right) p\left(x_{0}\right) \prod_{j=1}^{k-1} p\left(x_{j} \mid x_{j-1}\right)}{\pi\left(x_{0: k-1} \mid y_{1: k}\right)} \frac{p\left(y_{k} \mid x_{k}\right) p\left(x_{k} \mid x_{k-1}\right)}{\pi\left(x_{k} \mid x_{0: k-1}, y_{1: k}\right)} \\
= & w\left(x_{0: k-1}\right) \frac{p\left(y_{k} \mid x_{k}\right) p\left(x_{k} \mid x_{k-1}\right)}{\pi\left(x_{k} \mid x_{0: k-1}, y_{1: k}\right)}
\end{aligned}
$$

One of the difficulties in implementing this algorithm in practice is that after a few time steps, most weights become small in comparison to a few which become nearly equal to unity. This implies that most of the computational effort gets wasted on trajectories which do not eventually contribute to the final estimate. This problem is widely discussed in the existing literature and one way to remedy this problem is to introduce the notion of an effective sample size [23] given by

$$
N_{\mathrm{eff}}=\frac{1}{\sum_{i=1}^{N}\left\{\tilde{w}_{i, 0: k}\right\}^{2}}
$$

It may be noted that if all weights are equal, $N_{\text {eff }}=N$ and if all weights excepting one are zero, $N_{\text {eff }}=1$. Thus, when the effective sample at any time step falls below a threshold $N_{\text {thres }}$, a step involving resampling is implemented with a view to mitigate the effect of depletion of samples.

Furthermore, the choice of the importance sampling density function plays a crucial role in the success of the algorithm. It has been shown [23] that $\pi\left(x_{k} \mid x_{i, 0: k-1, y_{1: k}}\right)=p\left(x_{k} \mid x_{i, k-1}, y_{k}\right)$ is the importance function that minimizes the variance of the non-normalized weights, $w_{i, k}^{*}$, conditioned upon $x_{i, 0: k-1}$ and $y_{1: k}$. This can be shown by noting that

$$
\begin{aligned}
E_{\pi}\left[w\left(x_{0: k}\right) \mid x_{i, 0: k-1}, y_{1: k}\right] & =\int \frac{p\left(y_{k} \mid x_{k}\right) p\left(x_{k} \mid x_{i, k-1}\right)}{\pi\left(x_{k} \mid x_{i, 0: k-1}, y_{1: k}\right)} \pi\left(x_{k} \mid x_{i, 0: k-1}, y_{1: k}\right) \mathrm{d} x_{k} \\
& =\int p\left(y_{k} \mid x_{k}\right) p\left(x_{k} \mid x_{i, k-1}\right) \mathrm{d} x_{k} \\
& =p\left(y_{k} \mid x_{i, k-1}\right) .
\end{aligned}
$$

Similarly, the conditional variance of the weights can be obtained by

$$
\operatorname{Var}_{\pi}\left[w\left(x_{0: k}\right) \mid x_{i, 0: k}, y_{1: k}\right]=E_{\pi}\left[\left\{\frac{p\left(y_{k} \mid x_{k}\right) p\left(x_{k} \mid x_{i, k-1}\right)}{\pi\left(x_{k} \mid x_{i, 0: k-1}, y_{1: k}\right)}-p\left(y_{k} \mid x_{i, k-1}\right)\right\}^{2}\right] \text {. }
$$

It follows that this variance will be zero when

$$
\pi\left(x_{k} \mid x_{i, 0: k-1}, y_{1: k}\right)=\frac{p\left(y_{k} \mid x_{k}\right) p\left(x_{k} \mid x_{i, k-1}\right)}{p\left(y_{k} \mid x_{i, k-1}\right)} .
$$

By noting that $p\left(y_{k} \mid x_{k}\right)=p\left(y_{k} \mid x_{k}, x_{i, 0: k-1}\right)$, one can write

$$
\begin{aligned}
\pi\left(x_{k} \mid x_{i, 0: k-1}, y_{1: k}\right) & =\frac{p\left(y_{k} \mid x_{k}, x_{i, 0: k-1}\right) p\left(x_{k} \mid x_{i, k-1}\right)}{p\left(y_{k} \mid x_{i, k-1}\right)}=\frac{p\left(x_{k}, y_{k} \mid x_{i, 0: k-1}\right) p\left(x_{k} \mid x_{i, k-1}\right)}{p\left(x_{k} \mid x_{i, k-1}\right) p\left(y_{k} \mid x_{i, k-1}\right)} \\
& =p\left(x_{k} \mid y_{k}, x_{i, k-1}\right) .
\end{aligned}
$$

In general, it is not feasible to deduce the ideal importance sampling density function $p\left(x_{k} \mid y_{k}, x_{i, k-1}\right)$. However, when the process and measurement equations assume to be of the form

$$
\begin{aligned}
& x_{k}=f\left(x_{k-1}\right)+v_{k}, \quad v_{k} \equiv N\left[\begin{array}{ll}
0_{n_{v} \times 1} & \Sigma_{v}
\end{array}\right], \\
& y_{k}=C x_{k}+w_{k}, \quad w_{k} \equiv N\left[\begin{array}{ll}
0_{n_{w} \times 1} & \Sigma_{w}
\end{array}\right] .
\end{aligned}
$$


it can be shown that the ideal importance density function is a normal density function with mean $m_{k}$ and covariance $\Sigma$ given by

$$
\begin{aligned}
m_{k} & =\Sigma\left(\Sigma_{v}^{-1} f\left(x_{k-1}\right)+C^{t} \Sigma_{w}^{-1} y_{k}\right), \\
\Sigma^{-1} & =\Sigma_{v}^{-1}+C^{t} \Sigma_{w}^{-1} C .
\end{aligned}
$$

It may be noted that by $x \equiv N(a, B)$ we indicate that $x$ is a vector of normal random variables with mean vector $a$ and covariance matrix $B$. Equations of the type (A.12) are very likely to be encountered in problems of structural system identification and, therefore, the above result is of notable significance. When dealing with more general forms of process and measurement equations, as in Eq. (14), suitable strategies need to be evolved in choosing the importance function. In the present study however, we limit our attention to application of this method to equations of the form (A.12).

Thus, in order to implement the filtering with SIS, the following steps are adopted:

1. Set $k=0$; draw samples $\left\{x_{i, 0}\right\}_{i=1}^{N}$ from $p\left(x_{0}\right)$.

2. Set $k=k+1$;

3. Sample $\left\{\hat{x}_{i, k}\right\}_{i=1}^{N}$ from $\pi\left(x_{k} \mid x_{i, 0: k-1}, y_{1: k}\right)$ and set $\hat{x}_{i, 0: k}=\left\{x_{i, 0: k-1}, \hat{x}_{i, k}\right\}$ for $i \in[1, N]$.

4. For $i \in[1, N]$ compute the importance weights

$$
w_{i, k}^{*}=w_{i, k-1}^{*} \frac{p\left(y_{k} \mid \hat{x}_{i, k}\right) p\left(\hat{x}_{i, k} \mid \hat{x}_{i, k-1}\right)}{\pi\left(\hat{x}_{i, k} \mid \hat{x}_{i, 0: k-1}, y_{1: k}\right)}
$$

5. For $i \in[1, N]$, normalize the weights

$$
\tilde{w}_{i, k}=\frac{w_{i, k}^{*}}{\sum_{j=1}^{N} w_{j, k}^{*}}
$$

6. Evaluate the expectations of interest, for instance,

$$
a_{k \mid k}=\sum_{i=1}^{N} x_{i, k} \tilde{w}_{i, k} ; \quad \sum_{k \mid k}=\sum_{i=1}^{N}\left(x_{i, k}-a_{k \mid k}\right)\left(x_{i, k}-a_{k \mid k}\right)^{t} \tilde{w}_{i, k}
$$

7. Evaluate the effective sample $N_{\text {eff }}$ using Eq. (A.7).

8. If $N_{\text {eff }}>N_{\text {thres }}$, set $x_{i, 0: k}=\hat{x}_{i, 0: k}$; go to step 2 if $k<n$, otherwise end.

9. If $N_{\text {eff }} \leqslant N_{\text {thres }}$, implement the following:

- For $i \in[1, N]$, sample an index $j(i)$ distributed according to the discrete distribution with $N$ elements satisfying $P[j(i)=l]=\tilde{w}_{l, k}$ for $l=1,2 \ldots, N$.

- For $i \in[1, N]$, set $x_{i, 0: k}=\tilde{x}_{j(i), 0: k}$ and $w_{i, k}=(1 / N)$.

- Go to step 2 if $k<n$; otherwise, end.

In implementing this method it is assumed that it is possible to analytically deduce $p\left(x_{k} \mid x_{k-1}\right)$ and $p\left(y_{k} \mid x_{k}\right)$ using Eq. (A.12).

\section{References}

[1] M.I. Friswell, J.E. Mottershead, Finite Element Model Updating in Structural Dynamics, Kluwer Academic Publishers, Dordrecht, 1996.

[2] L. Ljung, System Identification: Theory for the User, Prentice-Hall Inc., New Jersey, 1997.

[3] D.J. Ewins, Modal Testing: Theory, Procedures and Applications, Research Studies Press, Badlock, 2000.

[4] R. Pintelon, J. Schoukens, System Identification: a Frequency Domain Approach, IEEE Press, New York, 2001.

[5] B. Peeters, G.D. Roeck, Stochastic system identification for operational modal analysis, ASME Journal of Dynamic Systems, Measurement and Control 123 (2001) 1-9. 
[6] N.A.L. Lieven, D.J. Ewins, Experimental modal analysis, theme issue, Philosophical Transactions of Royal Society of London, Series A 359 (2001) 1-219.

[7] H. Imai, C.B. Yun, O. Maruyama, M. Shinozuka, Fundamentals of system identification in structural dynamics, Probabilistic Engineering Mechanics 4 (4) (1989) 162-173.

[8] J.S. Bendat, Nonlinear Systems Techniques and Applications, Wiley, New York, 1998.

[9] M. Imregun, A survey of nonlinear analysis tools for structural systems, Shock and Vibration Digest 30 (5) (1998) $363-369$.

[10] R. Ghanem, M. Shinozuka, Structural system identification I: Theory, Journal of Engineering mechanics, ASCE 121 (2) (1995) 255-264.

[11] K. Worden, G.P. Tomlinson, Nonlinearity in Structural Dynamics: Detection, Identification and Modeling, IOP Publishing, London, 2001.

[12] K. Worden, G. Manson, Nonlinearity in experimental modal analysis, Philosophical Transaction of Royal Society of London, Series A 359 (2001) 113-130.

[13] R.A. Ibrahim, C.L. Pettit, Uncertainties and dynamic problems of bolted joints and other fasteners, Journal of Sound and Vibration 279 (3-5) (2005) 857-936.

[14] G. Kerschen, K. Worden, A.F. Vakakis, J.C. Golinval, Past, present and future of nonlinear system identification in structural dynamics, Mechanical Systems and Signal Processing 20 (3) (2006) 505-592.

[15] R.E. Kalman, A new approach to linear filtering and prediction problems, Transactions of ASME, Journal of Basic Engineering 82 (Series D) (1960) 35-45.

[16] T. Kailath, Lectures on Wiener and Kalman filtering, Verlag, New York, 1981.

[17] R.G. Brown, P.Y.C. Hwang, Introduction to random signals and applied Kalman filtering, second ed., Wiley, New York, 1992.

[18] M.S. Grewal, A.P. Andrews, Kalman Filtering: Theory and Practice using Matlab, Wiley, New York, 2001.

[19] C.K. Chui, G. Chen, Kalman Filtering with Real Time Applications, Springer, New York, 1998.

[20] C.B. Yun, M. Shinozuka, Identification of nonlinear structural dynamic systems, Journal of Structural Mechanics 8 (2) (1980) 187-203.

[21] N.J. Gordon, D.J. Salmond, A.F.M. Smith, Novel approach to nonlinear/non-Gaussian Bayesian state estimation, IEE ProceedingsF 140 (2) (1993) 107-113.

[22] H. Tanizaki, Nonlinear Filters: Estimation and Applications, second ed., Verlag, Berlin, 1996.

[23] A. Doucet, On sequential simulation-based methods for Bayesian filtering, Technical report CUED/F-INFENG/TR.310 (1998), Department of Engineering, University of Cambridge, UK, 1998.

[24] J.S. Liu, R. Chen, Sequential Monte Carlo methods for dynamical systems, Journal of the American Statistical Association 93 (1998) 1032-1044.

[25] M.K. Pitt, N. Shephard, Filtering via simulation: auxiliary particle filter, Journal of American Statistical Association 94 (1999) $59-599$.

[26] A. Doucet, S. Godsill, C. Andrieu, On sequential Monte Carlo sampling methods for Bayesian filtering, Statistics and Computing 10 (2000) 197-208.

[27] Y. Iba, Population Monte Carlo Algorithms, Transactions of Japanese Society of Artificial Intelligence 16 (2) (2001) $279-286$.

[28] A. Doucet, N. de Freitas, N. Gordon, Sequential Monte Carlo Methods in Practice, Springer, New York, 2001.

[29] D. Crisan, A. Doucet, A survey of convergence results on particle filtering methods for practioners, IEEE Transaction on Signal Processing 50 (3) (2002) 736-746.

[30] B. Ristic, S. Arulampallam, N. Gordon, Beyond the Kalman Filter: Particle Filters for Tracking Applications, Artech House, Boston, 2004.

[31] C.S. Manohar, D. Roy, Monte Carlo filters for identification of nonlinear systems, Sadhana 31 (4) (2006) $399-427$.

[32] J. Ching, J.L. Beck, K.A. Porter, Bayesian state and parameter estimation of uncertain dynamical systems, Probabilistic Engineering Mechanics 21 (1) (2006) 81-96.

[33] R. Sajeeb, C.S. Manohar, D. Roy, Rao-Blackwellization with substructuring for state and parameter estimations of a class of nonlinear dynamical systems, under review, Journal of Applied Mechanics, Transactions of ASME (2007).

[34] G. Kerschen, J.C. Golnival, F.M. Hemez, Bayesian model screening for the identification of nonlinear mechanical systems, Journal of Vibration and Acoustics, Transactions of ASME 125 (2003) 389-397.

[35] D.T. Magill, Optimal adaptive estimation of sampled stochastic processes, IEEE Transactions on Automatic Control AC 10 (4) (1965) 434-439.

[36] P.E. Kloeden, E. Platen, Numerical Solution of Stochastic Differential Equations, Springer, Berlin, 1999.

[37] J. Liu, M. West, Combined parameter and state estimation in simulation-based filtering, in: A. Doucet, N. de Freitas, N. Gordon (Eds.), Sequential Monte Carlo Methods in Practice, Springer, New York, 2001. 